\title{
On global multidimensional supersonic flows with vacuum states at infinity
}

\author{
Xu Gang ${ }^{1}$, Yin Huicheng ${ }^{2 *}$ \\ 1. Faculty of Science, Jiangsu University, Zhenjiang, Jiangsu 212013, China. \\ 2. Department of Mathematics and IMS, Nanjing University, Nanjing 210093, China.
}

\begin{abstract}
In this paper, we are concerned with the global existence and stability of a smooth supersonic flow with vacuum state at infinity in a 3-D infinitely long divergent nozzle. The flow is described by a 3-D steady potential equation, which is multi-dimensional quasilinear hyperbolic (but degenerate at infinity) with respect to the supersonic direction, and whose linearized part admits the form $\partial_{t}^{2}-\frac{1}{(1+t)^{2(\gamma-1)}}\left(\partial_{1}^{2}+\partial_{2}^{2}\right)+\frac{2(\gamma-1)}{1+t} \partial_{t}$ for $1<\gamma<2$. From the physical point of view, due to the expansive geometric property of the divergent nozzle and the mass conservation of gas, the moving gas in the nozzle will gradually become rarefactive and tends to a vacuum state at infinity, which implies that such a smooth supersonic flow should be globally stable for small perturbations since there are no strong resulting compressions in the motion of the flow. We will confirm such a global stability phenomena by rigorous mathematical proofs and further show that there do not exist vacuum domains in any finite part of the nozzle.
\end{abstract}

Keywords: Supersonic flow, divergent nozzle, vacuum, anisotropic weighted energy estimate, global existence

Mathematical Subject Classification 2000: 35L70, 35L65, 35L67, 76N15

\section{$\S 1$ Introduction and main results}

In this paper, we are concerned with the global existence and stability of a smooth supersonic polytropic gas with vacuum state at infinity in a 3-D infinitely long divergent nozzle. The divergent nozzle is described by the domain $\Omega=\left\{x=\left(x_{1}, x_{2}, x_{3}\right) \in \mathbb{R}^{3}: x_{1}^{2}+x_{2}^{2} \leq\right.$ $\left.\tan ^{2} \varphi_{0} x_{3}^{2}, x_{1}^{2}+x_{2}^{2}+x_{3}^{2} \geq 1, x_{3}>0\right\}$ with $\varphi_{0} \in\left(0, \frac{\pi}{2}\right)$ (see the Figure 1 below), and the potential function $\Phi$ of irrotational polytropic gas satisfies the following steady potential equation

\footnotetext{
${ }^{*} \mathrm{Xu}$ Gang was supported by the National Natural Science Foundation of China (No.11101190) and Natural Science Fundamental Research Project of Jiangsu Colleges (No.10KLB110002); Yin Huicheng was supported by the NSFC (No. 10931007, No. 11025105) and the Priority Academic Program Development of Jiangsu Higher Education Institutions.
} 
in $\Omega$ :

$$
\sum_{i=1}^{3}\left(\left(\partial_{i} \Phi\right)^{2}-c^{2}(\rho)\right) \partial_{i}^{2} \Phi+2 \sum_{1 \leq i<j \leq 3} \partial_{i} \Phi \partial_{j} \Phi \partial_{i j}^{2} \Phi=0
$$

where $\partial_{i}=\partial_{x_{i}}(1 \leq i \leq 3), c(\rho)=\sqrt{P^{\prime}(\rho)}$ is the local sound speed, $P(\rho)$ is the pressure, $\rho$ is the density, and state equation is given by $P(\rho)=\rho^{\gamma}$ with $1<\gamma<2$ (for the air, $\gamma \approx 1.4$ ). Moreover, the density $\rho=\rho\left(\nabla_{x} \Phi\right)$ can be determined by the Bernoulli's law:

$$
\frac{1}{2}\left|\nabla_{x} \Phi\right|^{2}+\frac{\gamma}{\gamma-1} \rho^{\gamma-1}=C_{0} \equiv \frac{1}{2} q_{0}^{2}+\frac{\gamma}{\gamma-1} \rho_{0}^{\gamma-1}
$$

where $\nabla_{x}=\left(\partial_{1}, \partial_{2}, \partial_{3}\right)$, and $q_{0}>c\left(\rho_{0}\right)$ (this means that the flow at the entrance is supersonic along the radial direction). Without loss of generality and for convenience, $C_{0}=1$ will be always assumed in the whole paper.

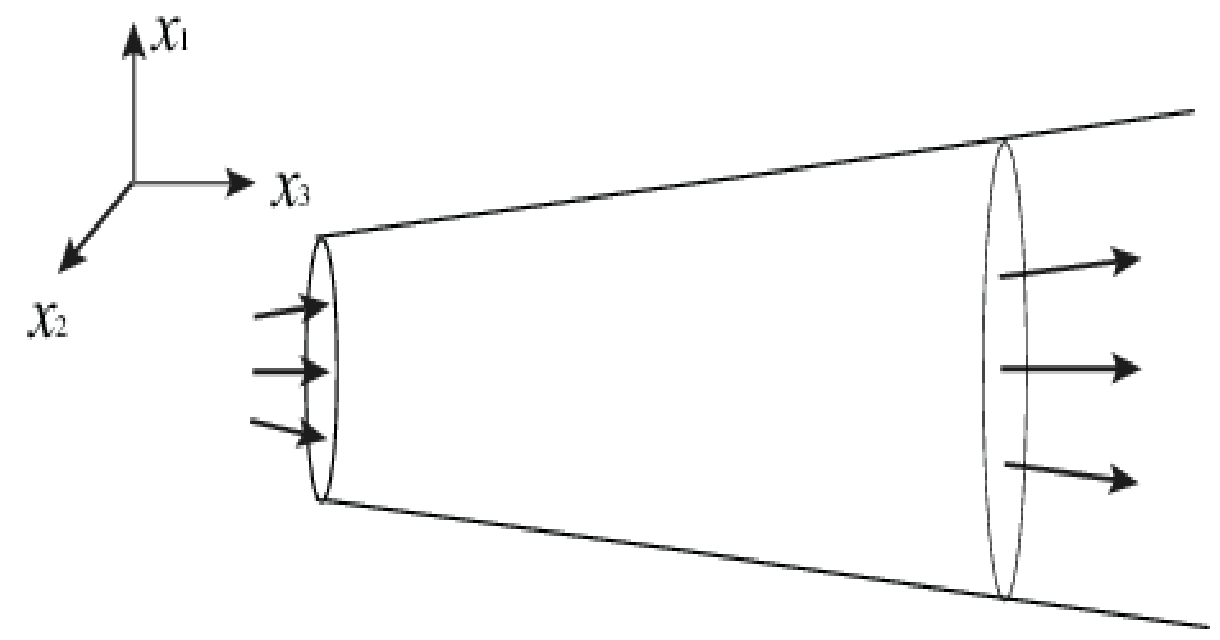

Figure 1. Supersonic flow in a 3-D divergent nozzle

Denote the divergent nozzle wall by $\Sigma=\left\{x: x_{1}^{2}+x_{2}^{2}=\tan ^{2} \varphi_{0} x_{3}^{2}, x_{1}^{2}+x_{2}^{2}+x_{3}^{2} \geq 1, x_{3}>\right.$ $0\}$, then $\Phi$ satisfies the following fixed boundary condition on $\Sigma$ :

$$
x_{1} \partial_{1} \Phi+x_{2} \partial_{2} \Phi-\tan ^{2} \varphi_{0} x_{3} \partial_{3} \Phi=0
$$

Due to the divergent geometric property of $\Omega$, it is convenient to work in the spherical coordinates $(r, \theta, \varphi)$ :

$$
\left(x_{1}, x_{2}, x_{3}\right)=(r \cos \theta \sin \varphi, r \sin \theta \sin \varphi, r \cos \varphi),
$$

where $r=\sqrt{x_{1}^{2}+x_{2}^{2}+x_{3}^{2}}, 0 \leq \theta \leq 2 \pi$ and $0 \leq \varphi \leq \varphi_{0}$.

Under the coordinate transformation (1.4), (1.1) becomes

$\left(\left(\partial_{r} \Phi\right)^{2}-c^{2}(\rho)\right) \partial_{r}^{2} \Phi+\frac{1}{r^{2} \sin ^{2} \varphi}\left(\frac{1}{r^{2} \sin ^{2} \varphi}\left(\partial_{\theta} \Phi\right)^{2}-c^{2}(\rho)\right) \partial_{\theta}^{2} \Phi+\frac{1}{r^{2}}\left(\frac{1}{r^{2}}\left(\partial_{\varphi} \Phi\right)^{2}-c^{2}(\rho)\right) \partial_{\varphi}^{2} \Phi$ 


$$
\begin{aligned}
& +\frac{2 \partial_{r} \Phi \partial_{\theta} \Phi}{r^{2} \sin ^{2} \varphi} \partial_{r \theta}^{2} \Phi+\frac{2}{r^{2}} \partial_{r} \Phi \partial_{\varphi} \Phi \partial_{r \varphi}^{2} \Phi+\frac{2 \partial_{\theta} \Phi \partial_{\varphi} \Phi}{r^{4} \sin ^{2} \varphi} \partial_{\theta \varphi}^{2} \Phi-\frac{1}{r^{3}}\left(2 r^{2} c^{2}(\rho)+\left(\partial_{\varphi} \Phi\right)^{2}\right. \\
& \left.+\frac{1}{\sin ^{2} \varphi}\left(\partial_{\theta} \Phi\right)^{2}\right) \partial_{r} \Phi-\frac{\cot \varphi}{r^{4}}\left(r^{2} c^{2}(\rho)+\frac{1}{\sin ^{2} \varphi}\left(\partial_{\theta} \Phi\right)^{2}\right) \partial_{\varphi} \Phi=0 .
\end{aligned}
$$

In particular, if the solution $\Phi$ of (1.5) is axially symmetric, namely, $\Phi(r, \theta, \varphi) \equiv \Phi(r, \varphi)$ is independent of the variable $\theta$, then (1.5) becomes

$$
\begin{gathered}
\left(\left(\partial_{r} \Phi\right)^{2}-c^{2}(\rho)\right) \partial_{r}^{2} \Phi+\frac{1}{r^{2}}\left(\frac{1}{r^{2}}\left(\partial_{\varphi} \Phi\right)^{2}-c^{2}(\rho)\right) \partial_{\varphi}^{2} \Phi+\frac{2}{r^{2}} \partial_{r} \Phi \partial_{\varphi} \Phi \partial_{r \varphi}^{2} \Phi \\
-\frac{1}{r^{3}}\left(2 r^{2} c^{2}(\rho)+\left(\partial_{\varphi} \Phi\right)^{2}\right) \partial_{r} \Phi-\frac{c^{2}(\rho)}{r^{2}} \cot \varphi \partial_{\varphi} \Phi=0 .
\end{gathered}
$$

Here we point out that some coefficients in (1.5) or (1.6) admit strong singularities near $\varphi=0$. Consequently, in order to overcome the difficulties arisen by the singularities near $\varphi=0$, we require to rewrite (1.5) or (1.6) by introducing some smooth vector fields tangent to the sphere $\mathbb{S}^{2}$ as in [15].

Set

$$
\left\{\begin{array}{l}
Z_{1}=x_{1} \partial_{2}-x_{2} \partial_{1}=\partial_{\theta} \\
Z_{2}=x_{2} \partial_{3}-x_{3} \partial_{2}=-\cot \varphi \cos \theta \partial_{\theta}-\sin \theta \partial_{\varphi}, \\
Z_{3}=x_{3} \partial_{1}-x_{1} \partial_{3}=-\cot \varphi \sin \theta \partial_{\theta}+\cos \theta \partial_{\varphi} .
\end{array}\right.
$$

Then it follows from a direct computation that (1.5) or (1.6) has such a new form

$$
\begin{aligned}
& \left(\left(\partial_{r} \Phi\right)^{2}-c^{2}(\rho)\right) \partial_{r}^{2} \Phi+\frac{2 \partial_{r} \Phi}{r^{2}} \sum_{i=1}^{3} Z_{i} \Phi \partial_{r} Z_{i} \Phi-\frac{c^{2}(\rho)}{r^{2}} \sum_{i=1}^{3} Z_{i}^{2} \Phi+\frac{1}{r^{4}} \sum_{i, j=1}^{3} Z_{i} \Phi Z_{j} \Phi Z_{i} Z_{j} \Phi \\
& +\sum_{i, j=1}^{3} \frac{C_{i j}(\omega)}{r^{3}} \partial_{r} \Phi Z_{i} \Phi Z_{j} \Phi+\sum_{i, j, k=1}^{3} \frac{C_{i j k}(\omega)}{r^{4}} Z_{i} \Phi Z_{j} \Phi Z_{k} \Phi-\frac{2 c^{2}(\rho)}{r} \partial_{r} \Phi=0
\end{aligned}
$$

where $\omega=\frac{x}{r}, C_{i j}(\omega)=C_{i j}\left(\frac{x}{r}\right)$ and $C_{i j k}(\omega)=C_{i j k}\left(\frac{x}{r}\right)$ are smooth functions on their arguments.

Meanwhile, the fixed boundary condition (1.3) can be changed as

$$
x_{1} Z_{3} \Phi-x_{2} Z_{2} \Phi=0 \quad \text { on } \Sigma .
$$

Especially, for the axially symmetric solution $\Phi$, the boundary condition on $\Sigma$ is

$$
Z_{2} \Phi=Z_{3} \Phi=0 \quad \text { on } \Sigma .
$$

In addition, we impose the following initial axially symmetric perturbations:

$$
\Phi(1, \theta, \varphi)=\varepsilon \Phi_{0}(\varphi), \quad \partial_{r} \Phi(1, \theta, \varphi)=q_{0}+\varepsilon \Phi_{1}(\varphi),
$$

where $\varepsilon>0$ is a small parameter, and $\Phi_{i}(\varphi) \in C_{0}^{\infty}\left[0, \varphi_{0}\right)(i=0,1)$. In fact, such kinds of initial conditions (1.11) can be easily realized by small axially symmetric perturbations on the initial density and velocity of irrotational gas. 


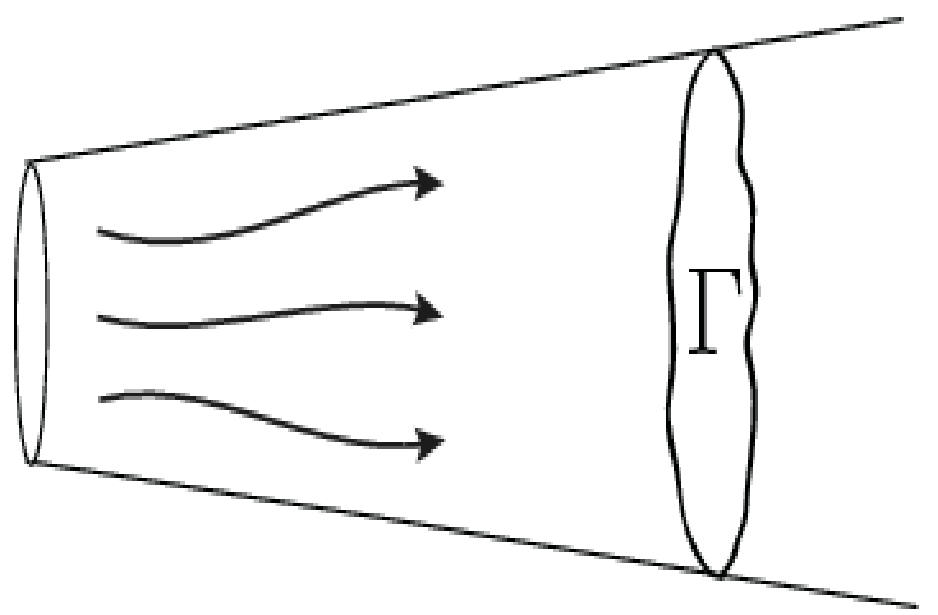

Figure 2. Perturbed supersonic flow in the 3-D divergent nozzle

Let $\Gamma=\left\{r=r(\theta, \varphi): 0 \leq \theta \leq 2 \pi, 0 \leq \varphi \leq \varphi_{0}\right\}$ be any $C^{1}$-smooth cross section of $\Omega$ (see the Figure 2 above). Denote the positive constant $m_{\varepsilon}=2 \pi \int_{0}^{\varphi_{0}} \rho_{0}^{\varepsilon}(\varphi)\left(q_{0}+\varepsilon \Phi_{1}(\varphi)\right) \sin \varphi d \varphi$, where the initial density $\rho_{0}^{\varepsilon}(\varphi)=\left(\frac{\gamma-1}{\gamma}\right)^{\frac{1}{\gamma-1}}\left\{\frac{\gamma}{\gamma-1} \rho_{0}^{\gamma-1}-\frac{1}{2}\left(2 q_{0} \varepsilon \Phi_{1}+\varepsilon^{2} \Phi_{1}^{2}+\varepsilon^{2}\left(\Phi_{0}^{\prime}\right)^{2}\right)\right\}^{\frac{1}{\gamma-1}}$ is determined by the Bernoulli's law (1.2). The main result in our paper is:

Theorem 1.1. There exists a constant $\varepsilon_{0}>0$ depending on $q_{0}, \rho_{0}$ and $\gamma$ such that problem (1.8) with (1.10)-(1.11) possesses a global $C^{\infty}$ supersonic solution $\Phi(x)$ for $\varepsilon<\varepsilon_{0}$ and the mass of gas on any smooth cross surface $\Gamma$ is conserved, namely, $\int_{\Gamma} \rho \nabla_{x} \Phi \cdot \vec{n} d S \equiv m_{\varepsilon}$, where $\vec{n}$ stands for the unit outward normal direction of $\Gamma$. Moreover, $\rho(x)>0$ and $\lim _{r \rightarrow \infty} \rho(x)=0$ hold in the whole $\Omega$.

Remark 1.1. From Theorem 1.1, one easily knows that there do not exist vacuum domains in any finite part of $\Omega$ for the problem (1.8) together with (1.10)-(1.11).

Remark 1.2. For the small arbitrarily (not axially symmetric) perturbed supersonic flow in $\Omega$, which is determined by the equation (1.8) together with (1.9) and the initial data $(\Phi(1, \theta, \varphi)$, $\left.\partial_{r} \Phi(1, \theta, \varphi)\right)=\left(\varepsilon \Phi_{0}(\theta, \varphi), q_{0}+\varepsilon \Phi_{1}(\theta, \varphi)\right)$ with $\Phi_{i}(\theta, \varphi) \in C_{0}^{\infty}\left([0,2 \pi] \times\left[0, \varphi_{0}\right)\right)(i=0,1)$, we can also solve the global stability problem as in Theorem 1.1 by analogous but much more complicated analysis. Nevertheless, due to the lengthy formulas and too heavy computations, we do not give out the related details of proof procedure here.

Remark 1.3. By the same analysis in this paper, Theorem 1.1 can be extended into the curved 2-D or 3-D divergent nozzles with small and arbitrary perturbations of straight boundaries (one can see the following Figure 3 and Figure 4). 


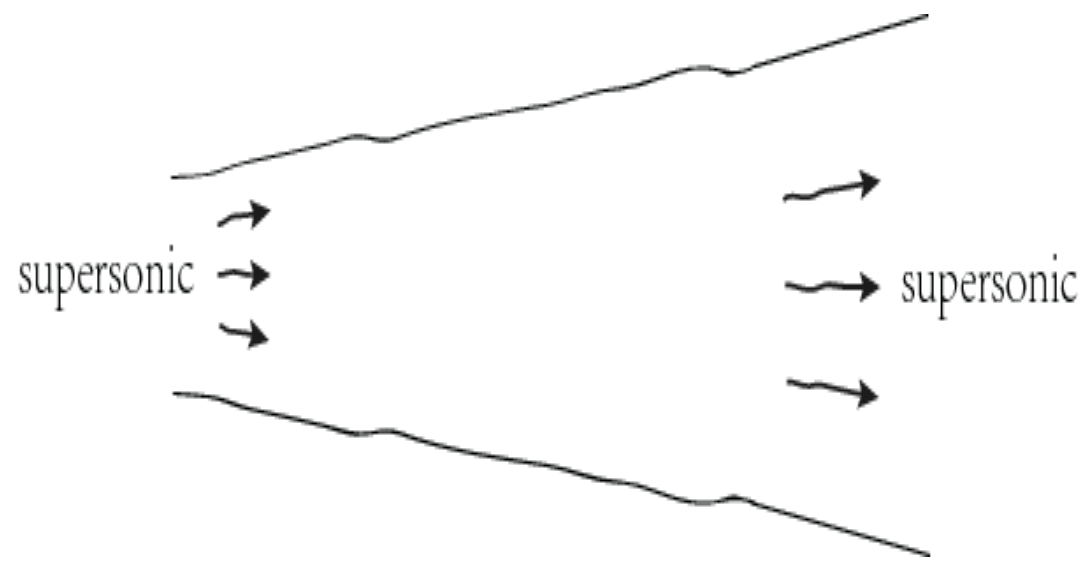

Figure 3. 2-D global smooth supersonic flow in a curved divergent nozzle

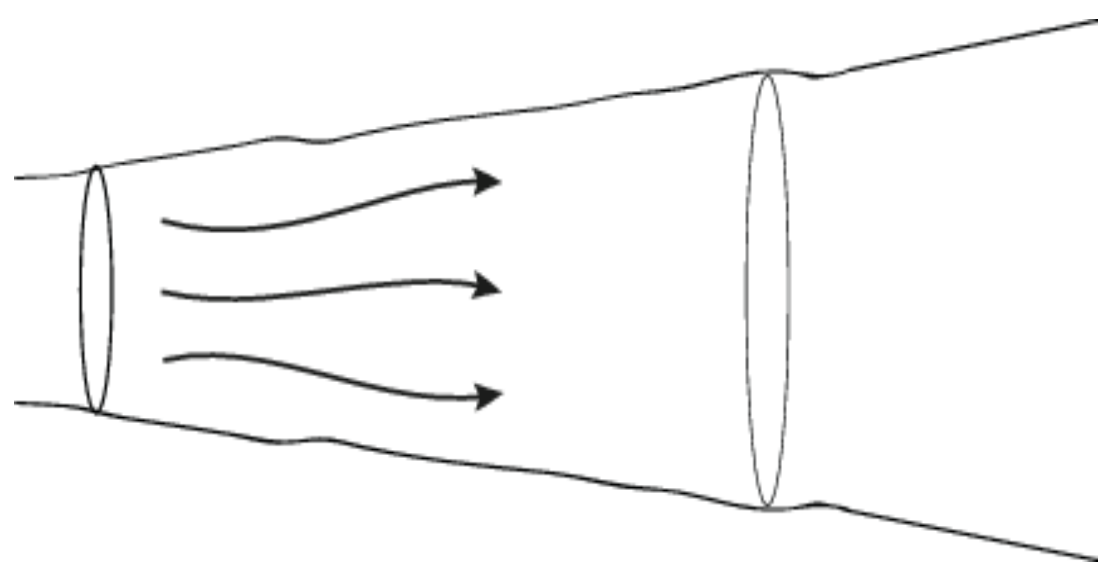

Figure 4. 3-D global smooth supersonic flow in a curved divergent nozzle

Remark 1.4. For the de Laval nozzle, which is constructed by a converging "entry" section and a diverging "exhaust" section, when the supersonic flow is formed across the sonic curve in the slowly variable nozzle and the infinite long nozzle walls approach two symmetric lines (see the Figure 5 below), then our Theorem 1.1 illustrates that the smooth supersonic flow exists globally for the small perturbed state. On the other hand, if the de Laval nozzle is finitely long and an appropriately large exit pressure $p_{e}$ is given, as stated in Section 147 of [5], at a certain place in the diverging part of the nozzle a shock front intervenes and the gas is compressed and slowed down to subsonic speed (see the Figure 6 below). This phenomenon has been extensively studied, especially the stability problem of a transonic shock is completely solved for a general class of 2-D de Laval nozzles whose divergent parts are small and arbitrary perturbations of divergent angular domains for the full steady compressible Euler system in [16]. 


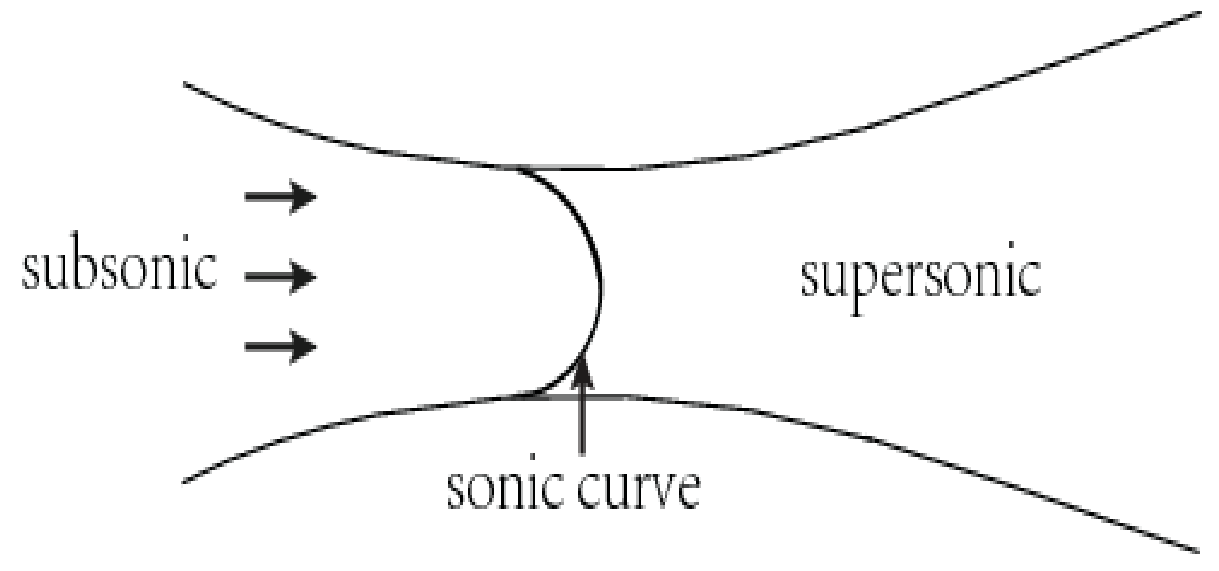

Figure 5. Global continuous transonic flow in an infinite long de Laval nozzle

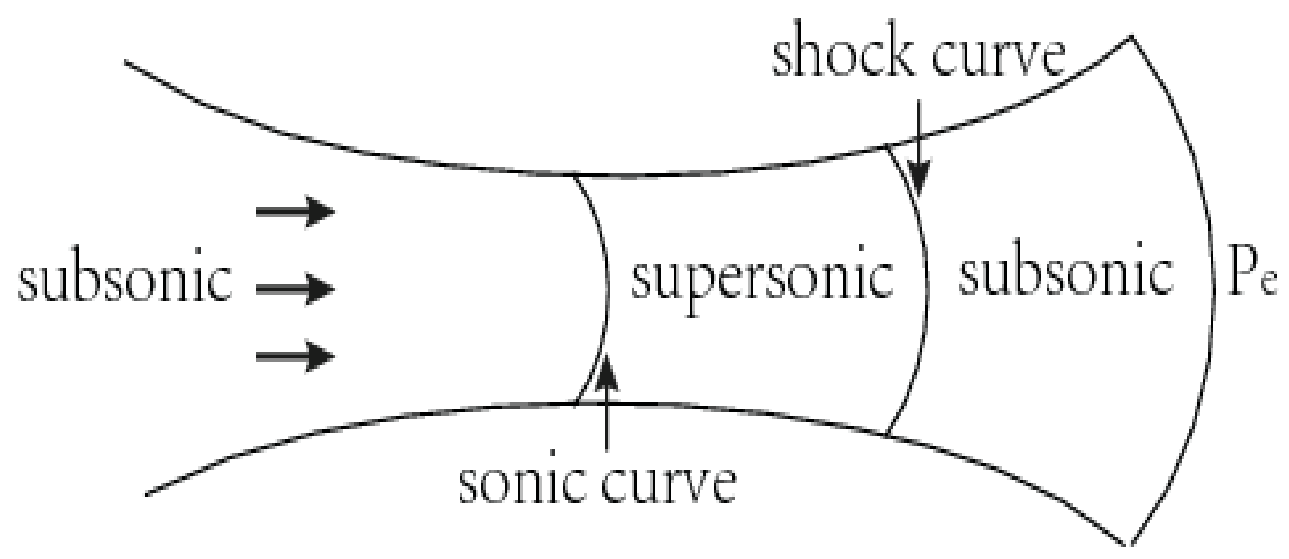

Figure 6. Stability of a transonic shock in a finitely long de Laval nozzle

Remark 1.5. The nonlinear equation (1.1) in our case is actually a two dimensional quasilinear degenerate wave equation if one regards $r$ as the time since the flow is supersonic in $r$-direction, whose linearized part is like $\partial_{t}^{2}-\frac{1}{(1+t)^{2(\gamma-1)}}\left(\partial_{1}^{2}+\partial_{2}^{2}\right)+\frac{2(\gamma-1)}{1+t} \partial_{t}$ (one can see Remark 3.1 below in §3). On the other hand, if we consider the Cauchy initial data problem of (1.1) which is of a small perturbation with respect to the uniform constant density $\rho_{0}$ and velocity $\left(0,0, q_{0}\right)$

$$
\left\{\begin{array}{l}
\sum_{i=1}^{3}\left(\left(\partial_{i} \Phi\right)^{2}-c^{2}(\rho)\right) \partial_{i}^{2} \Phi+2 \sum_{1 \leq i<j \leq 3} \partial_{i} \Phi \partial_{j} \Phi \partial_{i j}^{2} \Phi=0, \quad x_{3} \geq 0 \\
\left.\Phi(x)\right|_{x_{3}=0}=\varepsilon \Phi_{0}\left(x_{1}, x_{2}\right),\left.\quad \partial_{3} \Phi(x)\right|_{x_{3}=0}=q_{0}+\varepsilon \Phi_{1}\left(x_{1}, x_{2}\right), \quad\left(x_{1}, x_{2}\right) \in \mathbb{R}^{2},
\end{array}\right.
$$

where $q_{0}>c\left(\rho_{0}\right)$, and $\Phi_{i}\left(x_{1}, x_{2}\right) \in C_{0}^{\infty}\left(\mathbb{R}^{2}\right)(i=0,1)$, then by a direct verification, one sees that (1.12) does not fulfill the "null-condition" put forward in [4] and [14]. Therefore, in terms of the extensive results of [1-2], [9], [21], [28] and so on, the classical solution to 
(1.12) will blow up for finite $x_{3}$. However, compared this blowup result with our Theorem 1.1, we obtain the global existence of a smooth solution to (1.12) together with the divergent nozzle wall condition due to the rarefactive property of supersonic gas.

Remark 1.6. If the initial density contains vacuum, the local well-posedness results of compressible Euler system have been extensively studied in [3], [6-7], [12-13], [18], [20], [2527] and so on. In the general case, such local classical solution will blow up in finite time as shown in [3], [25] and the references therein. With respect to the problem in our paper, the vacuum only appears at infinity and the smooth solution exists globally.

Remark 1.7. If the initial velocity $u_{0}(x)$ of gas forces particles to spread out, roughly speaking, $u_{0}(x)$ is close to a linear field, which means $\varlimsup_{|x| \rightarrow \infty}\left|u_{0}(x)\right|=\infty$, the authors in [11] and [22] have proved the global existence of smooth solutions to the Cauchy problem of compressible Euler system. Here we emphasize that our initial data (1.11) are not the cases posed in [11] or [22] (for example, one can see Theorem 1 of [11]).

Let us comment on the proof of Theorem 1.1. Since the local solvability of problem (1.8) together with (1.10)-(1.11) has been known as long as the vacuum does not appear, we will use the continuous induction method to prove Theorem 1.1. To achieve this objective, we need to establish the global energy estimates with suitable anisotropic weights for (1.8) with (1.10)-(1.11), which is degenerate at infinity and admits a linear part as follows: $\partial_{r}^{2}-\frac{1}{(1+r)^{2(\gamma-1)}}\left(\partial_{1}^{2}+\partial_{2}^{2}\right)+\frac{2(\gamma-1)}{1+r} \partial_{r}$. Based on such estimates, one then obtains the absence of vacuum for any finite $x_{3}>0$ in $\Omega$, the global existence, stability, and the asymptotic behavior of the solution. The key ingredients in the analysis to obtain weighted energy estimates are to look for an appropriate multiplier and the suitable anisotropic weights, derive available boundary conditions of higher order derivatives of $\Phi$ on the boundary $\Sigma$ and search for the required weighted Sobolev interpolations. Finding a suitable multiplier and anisotropic weights are not easy due to the following reasons: Firstly, to obtain the global existence with no vacuum state requires to establish the estimates independent of $x_{3}$ and $\nabla_{x}^{\alpha} \Phi(0 \leq|\alpha| \leq 4)$ on the boundaries as well as in the interior of the domain $\Omega$. This leads to strict constraints on the multiplier and anisotropic weights, as well as makes the computations delicate and involved. Secondly, as our background solution tends to vacuum at infinity with different rates for the density and velocity and their derivatives respectively, one needs to take some measures to simplify the coefficients of the nonlinear equation (1.8) so that the procedure to find the multiplier and anisotropic weights and meanwhile avoid the appearance of vacuum for finite $x_{3}$ becomes manageable (one can see more detailed explanations in Remark 2.5 and $\$ 5$ below). Thirdly, the Neumann-type boundary condition (1.10) fulfilled by $\Phi$ arises additional difficulties since there are no enough information on $\Phi$ itself and its higher order derivatives. Thanks to some delicate analysis on the radial derivatives and angular derivatives of $\Phi$, which are closely accompanied by the weighted Sobolev interpolation inequalities in [17], we finally overcome all these difficulties and obtain a uniform weighted estimate of $\Phi$ and its higher-order derivatives with no vacuum state for any finite $x_{3}>0$ in $\Omega$. This eventually establishes Theorem 1.1.

This paper is organized as follows. In $\S 2$, we derive some basic estimates on the background solution with vacuum at infinity, and show some preliminary results regarding the weighted Sobolev interpolation inequalities. In $\S 3$, we reformulate problem (1.8) together with (1.10)- 
(1.11) by decomposing its solution as a sum of the background solution and a small perturbation $\dot{\Phi}$ so that its linearization can be studied in a convenient way. In $\S 4$, we will establish a uniform weighted energy estimate for the corresponding linear problem, where an appropriate multiplier is constructed. In $\S 5$, the uniform higher-order weighted estimates of $\dot{\Phi}$ are established by rather delicate analysis on the radial derivatives and angular derivatives of $\dot{\Phi}$, where the domain composition techniques are applied in order to obtain the energy estimates of $\dot{\Phi}$ near $\varphi=0$. In $\S 6$, based on the results in $\S 5$, we complete the proof of Theorem 1.1 by applying Sobolev's embedding theorem and continuous induction method.

\section{$\S 2$. Background solutions and some preliminaries}

In this section, at first we analyze the background solution to (1.6) with (1.10)-(1.11) when the initial data (1.11) are replaced by

$$
\left(\Phi(1, \theta, \varphi), \quad \partial_{r} \Phi(1, \theta, \varphi)\right)=\left(0, q_{0}\right) .
$$

In this case, the density $\rho(x)$ and velocity $u(x)=\nabla_{x} \Phi(x)$ in $\Omega$ have such forms: $\rho(x)=\hat{\rho}(r)$, $u(x)=\frac{x}{r} \hat{U}(r)$. Consequently, the problem (1.6) with (1.10) and (2.1) is equivalent to

$$
\left\{\begin{array}{l}
\left(r^{2} \hat{\rho} \hat{U}\right)^{\prime}(r)=0, \quad r \geq 1, \\
\frac{1}{2} \hat{U}^{2}(r)+\frac{\gamma}{\gamma-1} \hat{\rho}^{\gamma-1}(r)=1, \quad r \geq 1, \\
\hat{\rho}(1)=\rho_{0}, \quad \hat{U}(1)=q_{0} .
\end{array}\right.
$$

With respect to problem (2.2), we have

Lemma 2.1. For $r \geq 1,(2.2)$ has a global smooth solution in $\Omega$ which satisfies

$$
\begin{aligned}
& \hat{\rho}(r)=O\left(r^{-2}\right)>0, \quad c^{2}(\hat{\rho}(r))=O\left(r^{2(1-\gamma)}\right), \quad \hat{U}(r)=\sqrt{2}+O\left(r^{2(1-\gamma)}\right), \\
& \hat{U}^{\prime}(r)=O\left(r^{1-2 \gamma}\right)>0 .
\end{aligned}
$$

Correspondingly, the potential function $\hat{\Phi}(r)=\int_{1}^{r} \hat{U}(s) d s$.

Remark 2.1. Lemma 2.1 states an interesting physical phenomenon: along the direction of increasing area, a supersonic flow is expanded and accelerated, meanwhile becomes more and more rarefactive in the divergent nozzle. This and more physical phenomena on the supersonic or subsonic flows in divergent or convergent nozzles can be found in Chapter V of [5].

Proof. It follows from the first equation and the initial data in (2.2) that

$$
r^{2} \hat{\rho}(r) \hat{U}(r)=\rho_{0} q_{0}
$$

This, together with the second equation in (2.2), yields

$$
\left\{\begin{array}{l}
\hat{\rho}^{\prime}(r)=-\frac{2 \rho_{0} q_{0} \hat{U}}{r^{3}\left(\hat{U}^{2}-c^{2}(\hat{\rho})\right)} \\
\hat{U}^{\prime}(r)=\frac{2 \hat{U}^{2}(\hat{\rho})}{r\left(\hat{U}^{2}-c^{2}(\hat{\rho})\right)}
\end{array}\right.
$$


Thus, $\hat{\rho}^{\prime}(r)<0, \hat{U}^{\prime}(r)>0$ and $\left(\hat{U}^{2}-c^{2}(\hat{\rho})\right)^{\prime}(r)>0$ hold as long as $\hat{U}^{2}-c^{2}(\hat{\rho})>0$ and $\hat{U}>0$. From this, we can also obtain $\left(\hat{U}^{2}-\hat{c}^{2}(\hat{\rho})\right)(r) \geq\left(\hat{U}^{2}-c^{2}(\hat{\rho})\right)(1)=q_{0}^{2}-c^{2}\left(\rho_{0}\right)>0$ and $\hat{U}(r) \geq q_{0}$. On the other hand, if we set $f_{1}(\hat{\rho}, \hat{U}, r)=r^{2} \hat{\rho} \hat{U}-\rho_{0} q_{0}$ and $f_{2}(\hat{\rho}, \hat{U}, r)=\frac{1}{2} \hat{U}^{2}+$ $\frac{\gamma}{\gamma-1} \hat{\rho}^{\gamma-1}-1$, then a direct computation yields $\frac{\partial\left(f_{1}, f_{2}\right)}{\partial(\hat{\rho}, \hat{U})}=r^{2}\left(\hat{U}^{2}-c^{2}(\hat{\rho})\right) \geq q_{0}^{2}-c^{2}\left(\rho_{0}\right)>0$. Thus, $\hat{\rho}(r)$ and $\hat{U}(r)$ in (2.2) exist globally for $r \geq 1$ by implicit function theorem. In addition, (2.3) can be directly obtained by (2.4) and the second equality in (2.2), and (2.5) respectively.

Next, we cite an important weighted Sobolev interpolation inequality in [17], which will be applied to prove some crucial weighted inequalities listed in Lemma 2.6 below.

Lemma 2.2. (see [17]) Suppose $s, \tau, p, \alpha, \beta, q, a$ are real numbers, and $j \geq 0, m>0$ are integers, satisfying

$$
\left\{\begin{array}{l}
p, q \geq 1, \frac{j}{m} \leq a \leq 1, s>0, \\
\frac{1}{s}+\frac{\tau}{n}>0, \frac{1}{p}+\frac{\alpha}{n}>0, \frac{1}{q}+\frac{\beta}{n}>0 \\
m-j-\frac{n}{p} \text { is not a nonnegative integer. }
\end{array}\right.
$$

There exists a positive constant $C$ such that the following inequality holds for all $v \in$ $C_{0}^{\infty}\left(\mathbb{R}^{n}\right)$ :

$$
\left.\left.|| x\right|^{\tau} \nabla_{x}^{j} v\right|_{L^{s}} \leq\left.\left.\left.\left. C|| x\right|^{\alpha} \nabla_{x}^{m} v\right|_{L^{p}} ^{a}|| x\right|^{\beta} v\right|_{L^{q}} ^{1-a}
$$

if and only if the following conditions hold:

$$
\begin{aligned}
& \frac{1}{s}+\frac{\tau-j}{n}=a\left(\frac{1}{p}+\frac{\alpha-m}{n}\right)+(1-a)\left(\frac{1}{q}+\frac{\beta}{n}\right) \quad \text { with } \tau \leq a \alpha+(1-a) \beta ; \\
& \text { if } \frac{1}{q}+\frac{\beta}{n}=\frac{1}{p}+\frac{\alpha-m}{n} \text {, then } \\
& a(\alpha-m)+(1-a) \beta+j \leq \tau ;
\end{aligned}
$$

if $a=\frac{j}{m}$, then

$$
\tau=a \alpha+(1-a) \beta
$$

Corollary 2.3. For the domain $\Omega$ defined in $\S 1.1$, if $u \in C^{m}(\bar{\Omega})$ and

$$
\left.u\right|_{r \geq T} \equiv 0,
$$

where $T>1$ is a constant, then we have

(i) (2.7) still holds under the restrictions (2.6)(2.8)-(2.10), moreover the constant $C$ in the right hand side of (2.7) does not depend on $T$. 
(ii) for $m=2,1<\gamma<2, \sigma>0$ and $0<\delta<4 \gamma$,

$$
\begin{aligned}
& \left|r^{\frac{2 \gamma+2 \sigma-1}{4}} \nabla_{x} u\right|_{L^{4}(\Omega)} \leq C\left|r^{\frac{2 \gamma-1}{2}} \nabla_{x}^{2} u\right|_{L^{2}(\Omega)}^{\frac{1}{2}}\left|r^{\sigma} u\right|_{L^{\infty}(\Omega)}^{\frac{1}{2}}, \\
& \left|r^{\frac{4 \gamma+1}{4}} \nabla_{x} u\right|_{L^{4}(\Omega)} \leq C\left|r^{\frac{2 \gamma+1}{2}} \nabla_{x}^{2} u\right|_{L^{2}(\Omega)}^{\frac{1}{2}}\left|r^{\gamma} u\right|_{L^{\infty}(\Omega)}^{\frac{1}{2}}, \\
& \left|r^{\frac{8 \gamma-7-\delta}{4}} \nabla_{x} u\right|_{L^{4}(\Omega)} \leq C\left|r^{\frac{4 \gamma-3-\delta}{2}} \nabla_{x}^{2} u\right|_{L^{2}(\Omega)}^{\frac{1}{2}}\left|r^{2(\gamma-1)} u\right|_{L^{\infty}(\Omega)}^{\frac{1}{2}}, \\
& \left|r^{\frac{8 \gamma-3-\delta}{4}} \nabla_{x} u\right|_{L^{4}(\Omega)} \leq C\left|r^{\frac{4 \gamma-1-\delta}{2}} \nabla_{x}^{2} u\right|_{L^{2}(\Omega)}^{\frac{1}{2}}\left|r^{2 \gamma-1} u\right|_{L^{\infty}(\Omega)}^{\frac{1}{2}},
\end{aligned}
$$

where the generic positive constant $C$ is independent of $T$.

Proof. (i) The proof is completely parallel to that of Lemma 2.2 (one can check the details in [17]), then we omit it here.

(ii) In (2.6)(2.8)-(2.10) of Lemma 2.2, set $s=4, p=2, q=\infty, a=\frac{1}{2}$ and $j=1, m=2$, one then concludes that:

(2.12) and (2.13) come from (2.7) and the choices of $\tau=\frac{2 \gamma+2 \sigma-1}{4}, \alpha=\frac{2 \gamma-1}{2}, \beta=\sigma$ and $\tau=\frac{4 \gamma+1}{4}, \alpha=\frac{2 \gamma+1}{2}, \beta=\gamma$ respectively;

(2.14) and (2.15) are derived from (2.7) by choosing $\tau=\frac{8 \gamma-7-\delta}{4}, \alpha=\frac{4 \gamma-3-\delta}{2}, \beta=$ $2(\gamma-1)$ and $\tau=\frac{8 \gamma-3-\delta}{4}, \alpha=\frac{4 \gamma-1-\delta}{2}, \beta=2 \gamma-1$ respectively.

In order to apply Lemma 2.2 or Corollary 2.3 to derive some weighted Sobolev inequalities in $\Omega$ without the restriction (2.11), we require to establish an extension result as follows:

Lemma 2.4. Set $D_{T}=\left\{(r, \theta, \varphi): 1<r<T, 0 \leq \theta \leq 2 \pi, 0 \leq \varphi<\varphi_{0}\right\}$ for $T>1$. If $u(x) \in C^{3}\left(\bar{D}_{T}\right)$ and $r^{\beta} \partial_{x}^{\alpha} u \in L^{2}\left(D_{T}\right)(|\alpha| \leq 3)$ with some $\beta \in \mathbb{R}$, then there exists an extension $E u \in C^{3}\left(\bar{D}_{\frac{9}{8} T}\right)$ of $u$ such that $E u=u$ in $D_{T},\left.E u\right|_{r \geq \frac{9}{8} T} \equiv 0$ and

$$
\left|r^{\beta} E u\right|_{L^{\infty}\left(D_{\frac{9}{8} T}\right)} \leq C\left|r^{\beta} u\right|_{L^{\infty}\left(D_{T}\right)}, \quad\left|r^{\beta} \nabla_{x}^{\alpha} E u\right|_{L^{2}\left(D_{\frac{9}{8} T}\right)} \leq C \sum_{|\nu| \leq|\alpha|}\left|r^{\beta-|\alpha|+|\nu|} \nabla_{x}^{\nu} u\right|_{L^{2}\left(D_{T}\right)},
$$

where $C>0$ is independent of $T$.

Proof. In terms of the geometric property of $D_{T}$, it is convenient to use the spherical coordinate to work. Denote by $\tilde{u}(r, \theta, \varphi)=u(r \cos \theta \sin \varphi, r \sin \theta \sin \varphi, r \cos \varphi)$. Let $\tilde{E}$ be an extension operator defined as follows:

$$
(\tilde{E} u)(r, \theta, \varphi)= \begin{cases}\tilde{u}(r, \theta, \varphi), & 1 \leq r \leq T, \\ \sum_{j=1}^{4} \lambda_{j} \tilde{u}(T+j(T-r), \theta, \varphi), & T<r \leq \frac{9}{8} T\end{cases}
$$

where $\sum_{j=1}^{4}(-j)^{k} \lambda_{j}=1$ for $k=0,1,2,3$.

Noticing that

$$
1 \leq \frac{r}{T+j(T-r)} \leq \frac{9}{4} \quad \text { for } T \leq r \leq \frac{9}{8} T \text { and } 0 \leq j \leq 3
$$


then a direct computation yields

$$
\left|r^{\beta} \tilde{E} u\right|_{L^{\infty}\left(D_{\frac{9}{8} T}\right)} \leq C\left|r^{\beta} u\right|_{L^{\infty}\left(D_{T}\right)}
$$

and

$$
\begin{aligned}
& \left|r^{\beta} \nabla_{x} \tilde{E} u\right|_{L^{2}\left(D_{\frac{9}{8} T}\right)}^{2} \leq\left|r^{\beta} \nabla_{x} u\right|_{L^{2}\left(D_{T}\right)}^{2}+\left|r^{\beta} \nabla_{x} \tilde{E} u\right|_{L^{2}\left(D_{\frac{9}{8} T} \backslash D_{T}\right)}^{2} \\
& \leq\left|r^{\beta} \nabla_{x} u\right|_{L^{2}\left(D_{T}\right)}^{2}+\left|r^{\beta}\left(\left(\partial_{r} \tilde{E} u\right)^{2}+\frac{1}{r^{2} \sin ^{2} \varphi}\left(\partial_{\theta} \tilde{E} u\right)^{2}+\frac{1}{r^{2}}\left(\partial_{\varphi} \tilde{E} u\right)^{2}\right)\right|_{L^{2}\left(D_{\frac{9}{8}} T D_{T}\right)}^{2} \\
& \leq\left|r^{\beta} \nabla_{x} u\right|_{L^{2}\left(D_{T}\right)}^{2}+C\left|r^{\beta}\left(\left(\partial_{r} u\right)^{2}+\frac{1}{r^{2} \sin ^{2} \varphi}\left(\partial_{\theta} u\right)^{2}+\frac{1}{r^{2}}\left(\partial_{\varphi} u\right)^{2}\right)\right|_{L^{2}\left(D_{T} \backslash D_{\frac{1}{2} T}\right)}^{2} \\
& \leq C\left|r^{\beta} \nabla_{x} u\right|_{L^{2}\left(D_{T}\right)}^{2} .
\end{aligned}
$$

Analogously, we have for $|\alpha| \leq 3$

$$
\left|r^{\beta} \nabla_{x}^{\alpha} \tilde{E} u\right|_{L^{2}\left(D_{\frac{9}{8} T}\right)} \leq C\left|r^{\beta} \nabla_{x}^{\alpha} u\right|_{L^{2}\left(D_{T}\right)} .
$$
setting

Choosing a $C^{\infty}$-smooth function $\eta(s)$ with $\eta(s) \equiv 1$ for $s \leq 1$ and $\eta(s) \equiv 0$ for $s \geq \frac{9}{8}$ and

$$
E u(x)=\eta\left(\frac{r}{T}\right) \tilde{E} u,
$$

then $E u$ satisfies (2.16) and the proof of Lemma 2.4 is completed.

Remark 2.2. From Lemma 2.4, we easily know that Corollary 2.3 still holds when the assumption (2.11) is removed.

With respect to the $Z$-fileds introduced in (1.7), we have the following properties by direct verifications as in [15].

Lemma 2.5.

(i) $\left[Z_{1}, Z_{2}\right]=Z_{3},\left[Z_{2}, Z_{3}\right]=Z_{1},\left[Z_{3}, Z_{1}\right]=Z_{2}$.

(ii) $\left[Z_{i}, \partial_{r}\right]=0, Z_{i} r=0$.

(iii) $\nabla_{x} f \cdot \nabla_{x} g=\partial_{r} f \cdot \partial_{r} g+\frac{1}{r^{2}} \sum_{i=1}^{3} Z_{i} f \cdot Z_{i} g$ for any $C^{1}$ smooth functions $f$ and $g$.

(iv) $|Z v| \leq r\left|\nabla_{x} v\right|$ for any $C^{1}$ smooth function $v$, here and below $Z \in\left\{Z_{1}, Z_{2}, Z_{3}\right\}$.

(v) $\quad \partial_{1}=\frac{x_{1}}{r} \partial_{r}+\frac{x_{2}}{r^{2}} Z_{1}-\frac{x_{3}}{r^{2}} Z_{3} ; \quad \partial_{2}=\frac{x_{2}}{r} \partial_{r}+\frac{x_{3}}{r^{2}} Z_{2}-\frac{x_{1}}{r^{2}} Z_{1} ; \quad \partial_{3}=\frac{x_{3}}{r} \partial_{r}+\frac{x_{1}}{r^{2}} Z_{3}-\frac{x_{2}}{r^{2}} Z_{2}$.

Remark 2.3. If $u \in C^{m}\left(\mathbb{R}^{3}\right)$ with $m \in \mathbb{N}$, then by Lemma 2.5 we have $\left|\nabla_{x}^{m} u\right| \sim\left|\partial_{r}^{m} u\right|+$ $\frac{\left|\partial_{r}^{m-1} Z u\right|}{r}+\frac{\left|\partial_{r}^{m-2} Z^{2} u\right|}{r^{2}}+\ldots+\frac{\left|Z^{m} u\right|}{r^{m}}$.

As direct applications of Remark 2.2 and Lemma 2.5, we have the following inequalities which will be used again and again in $\S 5$ below. 
Lemma 2.6. If $1<\gamma<2, \sigma \geq \gamma-1,0<\delta<4 \gamma, u(x) \in C^{4}\left(\bar{D}_{T}\right)$, then there exists a generic positive constant $C$ independent of $T$ such that

(i) $\quad\left|r^{\frac{2 \gamma+2 \sigma-9}{4}} Z^{2} u\right|_{L^{4}\left(D_{T}\right)} \leq C\left(\sum_{k=0}^{2}\left|r^{\frac{2 \gamma-1}{2}-k} \nabla_{x}^{2-k}\left(\frac{1}{r} Z u\right)\right|_{L^{2}\left(D_{T}\right)}^{\frac{1}{2}}\right)\left|r^{\sigma-1} Z u\right|_{L^{\infty}\left(D_{T}\right)}^{\frac{1}{2}}$.

(ii) $\quad\left|r^{\frac{4 \gamma-11}{4}} Z^{3} u\right|_{L^{4}\left(D_{T}\right)} \leq C\left(\sum_{k=0}^{2}\left|r^{\frac{2 \gamma+1}{2}-k} \nabla_{x}^{3-k}\left(\frac{1}{r} Z u\right)\right|_{L^{2}\left(D_{T}\right)}^{\frac{1}{2}}\right)\left|r^{\gamma} \nabla_{x}\left(\frac{1}{r} Z u\right)\right|_{L^{\infty}\left(D_{T}\right)}^{\frac{1}{2}}$

(iii) $\quad\left|r^{\frac{8 \gamma-11-\delta}{4}} \partial_{r} Z^{2} u\right|_{L^{4}\left(D_{T}\right)}+\left|r^{\frac{8 \gamma-7-\delta}{4}} \partial_{r}^{2} Z u\right|_{L^{4}\left(D_{T}\right)}$

$$
\leq C\left(\sum_{k=0}^{2}\left|r^{\frac{4 \gamma-3-\delta}{2}-k} \nabla_{x}^{2-k} \partial_{r} Z u\right|_{L^{2}\left(D_{T}\right)}^{\frac{1}{2}}\right)\left|r^{2(\gamma-1)} \partial_{r} Z u\right|_{L^{\infty}\left(D_{T}\right)}^{\frac{1}{2}}
$$

(iv) $\quad\left|r^{\frac{8 \gamma-3-\delta}{4}} \partial_{r}^{3} u\right|_{L^{4}\left(D_{T}\right)} \leq C\left(\sum_{k=0}^{2}\left|r^{\frac{4 \gamma-1-\delta}{2}-k} \nabla_{x}^{2-k} \partial_{r}^{2} u\right|_{L^{2}\left(D_{T}\right)}^{\frac{1}{2}}\right)\left|r^{2 \gamma-1} \partial_{r}^{2} u\right|_{L^{\infty}\left(D_{T}\right)}^{\frac{1}{2}}$.

Proof. Let $E$ be the extension operator given in Lemma 2.4, then we have

(i)

$$
\begin{aligned}
& \left|r^{\frac{2 \gamma+2 \sigma-9}{4}} Z^{2} u\right|_{L^{4}\left(D_{T}\right)} \\
\leq & C\left|r^{\frac{2 \gamma+2 \sigma-1}{4}} \nabla_{x}\left(\frac{1}{r} Z u\right)\right|_{L^{4}\left(D_{T}\right)} \quad \text { (Applying } \frac{Z^{2}}{r^{2}}=\frac{Z}{r}\left(\frac{Z}{r}\right) \text { due to Lemma 2.5 (ii)) } \\
\leq & C\left|r^{\frac{2 \gamma+2 \sigma-1}{4}} \nabla_{x} E\left(\frac{1}{r} Z u\right)\right|_{L^{4}(\Omega)} \\
\leq & \left.C\left|r^{\frac{2 \gamma-1}{2}} \nabla_{x}^{2} E\left(\frac{1}{r} Z u\right)\right|_{L^{2}(\Omega)}^{\frac{1}{2}}\left|r^{\sigma} E\left(\frac{1}{r} Z u\right)\right|_{L^{\infty}(\Omega)}^{\frac{1}{2}} \quad \text { (Applying (2.12) for } E\left(\frac{1}{r} Z u\right)\right) \\
\leq & C\left(\sum_{k=0}^{2}\left|r^{\frac{2 \gamma-1}{2}-k} \nabla_{x}^{2-k}\left(\frac{1}{r} Z u\right)\right|_{L^{2}\left(D_{T}\right)}^{\frac{1}{2}}\right)\left|r^{\sigma-1} Z u\right|_{L^{\infty}\left(D_{T}\right)}^{\frac{1}{2}} \quad \text { (By Lemma 2.4) }
\end{aligned}
$$

(ii)

$$
\begin{aligned}
& \left|r^{\frac{4 \gamma-11}{4}} Z^{3} u\right|_{L^{4}\left(D_{T}\right)} \\
\leq & C\left|r^{\frac{4 \gamma+1}{4}} \nabla_{x}^{2}\left(\frac{1}{r} Z u\right)\right|_{L^{4}\left(D_{T}\right)}+C\left|r^{\frac{4 \gamma-3}{4}} \nabla_{x}\left(\frac{1}{r} Z u\right)\right|_{L^{4}\left(D_{T}\right)} \\
\leq & C\left|r^{\frac{4 \gamma+1}{4}} \nabla_{x} E\left(\nabla_{x}\left(\frac{1}{r} Z u\right)\right)\right|_{L^{4}(\Omega)}+C\left|r^{\frac{2 \gamma+2 \sigma-1}{4}} \nabla_{x}\left(\frac{1}{r} Z u\right)\right|_{L^{4}\left(D_{T}\right)} \quad(\text { By } \sigma \geq \gamma-1) \\
\leq & \left.C\left|r^{\frac{2 \gamma+1}{2}} \nabla_{x}^{2} E\left(\nabla_{x}\left(\frac{1}{r} Z u\right)\right)\right|_{L^{2}(\Omega)}^{\frac{1}{2}}\left|r^{\gamma} E\left(\nabla_{x}\left(\frac{1}{r} Z u\right)\right)\right|_{L^{\infty}(\Omega)}^{\frac{1}{2}} \quad \quad \text { (Applying (2.13) for } E\left(\nabla_{x}\left(\frac{1}{r} Z u\right)\right)\right) \\
& C\left|r^{\frac{2 \gamma+2 \sigma-1}{4}} \nabla_{x}\left(\frac{1}{r} Z u\right)\right|_{L^{4}\left(D_{T}\right)}
\end{aligned}
$$




$$
\begin{aligned}
\leq & C\left(\sum_{k=0}^{2}\left|r^{\frac{2 \gamma+1}{2}-k} \nabla_{x}^{3-k}\left(\frac{1}{r} Z u\right)\right|_{L^{2}\left(D_{T}\right)}^{\frac{1}{2}}\right)\left|r^{\gamma} \nabla_{x}\left(\frac{1}{r} Z u\right)\right|_{L^{\infty}\left(D_{T}\right)}^{\frac{1}{2}} \\
& +C\left(\sum_{k=0}^{2}\left|r^{\frac{2 \gamma-1}{2}-k} \nabla_{x}^{2-k}\left(\frac{1}{r} Z u\right)\right|_{L^{2}\left(D_{T}\right)}^{\frac{1}{2}}\right)\left|r^{\sigma-1} Z u\right|_{L^{\infty}\left(D_{T}\right)}^{\frac{1}{2}} \quad \text { (By Lemma } 2.4 \text { and (i)) }
\end{aligned}
$$

(iii)

$$
\begin{aligned}
& \left|r^{\frac{8 \gamma-11-\delta}{4}} \partial_{r} Z^{2} u\right|_{L^{4}\left(D_{T}\right)}+\left|r^{\frac{8 \gamma-7-\delta}{4}} \partial_{r}^{2} Z u\right|_{L^{4}\left(D_{T}\right)} \\
\leq & C\left|r^{\frac{8 \gamma-7-\delta}{4}} \nabla_{x}\left(\partial_{r} Z u\right)\right|_{L^{4}\left(D_{T}\right)} \\
\leq & C\left|r^{\frac{8 \gamma-7-\delta}{4}} \nabla_{x} E\left(\partial_{r} Z u\right)\right|_{L^{4}(\Omega)} \\
\leq & C\left|r^{\frac{4 \gamma-3-\delta}{2}} \nabla_{x}^{2} E\left(\partial_{r} Z u\right)\right|_{L^{2}(\Omega)}^{\frac{1}{2}}\left|r^{2(\gamma-1)} E\left(\partial_{r} Z u\right)\right|_{L^{\infty}(\Omega)}^{\frac{1}{2}} \quad\left(\text { Applying (2.14) for } E\left(\partial_{r} Z u\right)\right) \\
\leq & C\left(\sum_{k=0}^{2}\left|r^{\frac{4 \gamma-3-\delta}{2}-k} \nabla_{x}^{2-k} \partial_{r} Z u\right|_{L^{2}\left(D_{T}\right)}^{\frac{1}{2}}\right)\left|r^{2(\gamma-1)} \partial_{r} Z u\right|_{L^{\infty}\left(D_{T}\right)}^{\frac{1}{2}} \quad \text { (By Lemma 2.4) }
\end{aligned}
$$

(iv)

$$
\begin{aligned}
& \left|r^{\frac{8 \gamma-3-\delta}{4}} \partial_{r}^{3} u\right|_{L^{4}\left(D_{T}\right)} \\
\leq & C\left|r^{\frac{8 \gamma-3-\delta}{4}} \nabla_{x}\left(\partial_{r}^{2} u\right)\right|_{L^{4}\left(D_{T}\right)} \\
\leq & C\left|r^{\frac{8 \gamma-3-\delta}{4}} \nabla_{x} E\left(\partial_{r}^{2} u\right)\right|_{L^{4}(\Omega)} \\
\leq & C\left|r^{\frac{4 \gamma-1-\delta}{2}} \nabla_{x}^{2} E\left(\partial_{r}^{2} u\right)\right|_{L^{2}(\Omega)}^{\frac{1}{2}}\left|r^{2 \gamma-1} E\left(\partial_{r}^{2} u\right)\right|_{L^{\infty}(\Omega)}^{\frac{1}{2}} \quad\left(\text { Applying (2.15) for } E\left(\partial_{r}^{2} u\right)\right) \\
\leq & C\left(\sum_{k=0}^{2}\left|r^{\frac{4 \gamma-1-\delta}{2}-k} \nabla_{x}^{2-k} \partial_{r}^{2} u\right|_{L^{2}\left(D_{T}\right)}^{\frac{1}{2}}\right)\left|r^{2 \gamma-1} \partial_{r}^{2} u\right|_{L^{\infty}\left(D_{T}\right)}^{\frac{1}{2}} \quad \text { (By Lemma 2.4) }
\end{aligned}
$$

Therefore, we complete the proof of Lemma 2.6.

Based on Lemma 2.6, we further have

Lemma 2.7. If $1<\gamma<2, \sigma=\min \{1,2(\gamma-1)\}, 0<\delta<4 \gamma, u(x) \in C^{4}\left(\bar{D}_{T}\right)$, and the following assumptions hold for some constant $M>0$

$$
\sum_{0 \leq l_{1}+l_{2} \leq 1} r^{l_{1}}\left|\partial_{r}^{l_{1}} Z^{l_{2}} \partial_{r} u\right| \leq M \varepsilon r^{-2(\gamma-1)}, \quad r^{-1}|Z u| \leq M \varepsilon r^{-\sigma}, \quad r^{-1}\left|Z^{2} u\right| \leq M \varepsilon r^{-(\gamma-1)}
$$

then

$$
\left|r^{\frac{2 \gamma+2 \sigma-9}{4}} Z^{2} u\right|_{L^{4}} \leq C(M) \varepsilon^{\frac{1}{2}}\left(\sum_{l=0}^{2}\left(\left|r^{\frac{4 \gamma-7-\delta+2 l}{2}} \nabla_{x}^{l} \partial_{r} u\right|_{L^{2}}+\left|r^{\frac{2 \gamma-5+2 l}{2}} \nabla_{x}^{l}\left(\frac{1}{r} Z u\right)\right|_{L^{2}}\right)\right)^{\frac{1}{2}}
$$


and

$$
\begin{aligned}
& \left|r^{\frac{4 \gamma-11}{4}} Z^{3} u\right|_{L^{4}}+\left|r^{\frac{8 \gamma-11-\delta}{4}} \partial_{r} Z^{2} u\right|_{L^{4}}+\left|r^{\frac{8 \gamma-7-\delta}{4}} \partial_{r}^{2} Z u\right|_{L^{4}}+\left|r^{\frac{8 \gamma-3-\delta}{4}} \partial_{r}^{3} u\right|_{L^{4}} \\
& \leq C(M) \varepsilon^{\frac{1}{2}}\left(\sum_{l=0}^{3}\left(\left|r^{\frac{4 \gamma-7-\delta+2 l}{2}} \nabla_{x}^{l} \partial_{r} u\right|_{L^{2}}+\left|r^{\frac{2 \gamma-5+2 l}{2}} \nabla_{x}^{l}\left(\frac{1}{r} Z u\right)\right|_{L^{2}}\right)\right)^{\frac{1}{2}}
\end{aligned}
$$

where $C(M)>0$ is a constant depending on $M$.

Remark 2.4. By $1<\gamma<2$ and $\sigma=\min \{1,2(\gamma-1)\}$, we can easily conclude $\sigma \geq \gamma-1$, which means that $\sigma$ satisfies the requirement in Lemma 2.6.

Remark 2.5. (2.21) actually comes from the induction assumptions in Theorem 5.1 on $\dot{\Phi}$, where $\dot{\Phi}$ is the difference between the solution $\Phi$ of (1.8) and the background solution $\hat{\Phi}$. By (2.21), we know that $\left|\partial_{r} u\right| \leq M \varepsilon r^{-2(\gamma-1)}$ but $\left|\frac{Z u}{r}\right| \leq M \varepsilon r^{-\sigma}$, and $\left|\partial_{r} Z u\right| \leq M \varepsilon r^{-2(\gamma-1)}$ but $\left|\frac{Z^{2} u}{r}\right| \leq M \varepsilon r^{-(\gamma-1)}$, which implies that the decay rates of the radial derivatives and angular derivatives of $u$ are different. Consequently, in order to obtain the anisotropic energy estimates of $\dot{\Phi}$ in $\S 5$, we have to pay much attentions on distinguishing the different roles of $\partial_{r} \dot{\Phi}$ and $Z \dot{\Phi}$, and this leads to rather involved and delicate analysis.

Proof. In order to prove (2.22)-(2.23), we only verify $\left|r^{\frac{2 \gamma+2 \sigma-9}{4}} Z^{2} u\right|_{L^{4}}$ to satisfy (2.22) since the terms in the left hand side of (2.23) can be analogously done.

It follows from Lemma 2.5, the assumptions on $\gamma$ and $\delta$, and a direct computation that

$$
\begin{aligned}
& \sum_{k=0}^{2}\left|r^{\frac{2 \gamma-1}{2}-k} \nabla_{x}^{2-k}\left(\frac{1}{r} Z u\right)\right|_{L^{2}\left(D_{T}\right)} \\
& \leq C \sum_{l=0}^{2}\left(\left|r^{\frac{4 \gamma-7-\delta+2 l}{2}}\left(\nabla_{x}^{l} \partial_{r} u\right)\right|_{L^{2}\left(D_{T}\right)}+\left|r^{\frac{2 \gamma-5+2 l}{2}}\left(\nabla_{x}^{l}\left(\frac{1}{r} Z u\right)\right)\right|_{L^{2}\left(D_{T}\right)}\right) .
\end{aligned}
$$

On the other hand, by (2.21) we have

$$
\left|r^{\sigma-1} Z u\right|_{L^{\infty}\left(D_{T}\right)} \leq M \varepsilon
$$

Consequently, by Lemma 2.6 (i), we know that (2.22) holds for $\left|r^{\frac{2 \gamma+2 \sigma-9}{4}} Z^{2} u\right|_{L^{4}}$, and then the proof of (2.23) can be completed similarly.

\section{$\S 3$. Reformulation of the problem (1.8) with (1.10)-(1.11)}

At first, we state a local solvability result on the problem (1.8) with (1.10)-(1.11).

Lemma 3.1. There exists a $T_{0}>1$ such that the problem (1.8) with (1.10)-(1.11) possesses a local $C^{\infty}$ solution $\Phi(r, \varphi)$ in $\Omega_{T_{0}}=\left\{(r, \theta, \varphi): 1 \leq r \leq T_{0}, 0 \leq \theta \leq 2 \pi, 0 \leq \varphi \leq \varphi_{0}\right\}$. Moreover, for any $k \in \mathbb{N} \cup\{0\}$, there exists a positive constant $C_{k}$ such that

$$
\|\Phi(r, \theta, \varphi)-\hat{\Phi}(r)\|_{C^{k}\left(\Omega_{T_{0}}\right)} \leq C_{k} \varepsilon
$$

where $\hat{\Phi}(r)$ is given in Lemma 2.1 . 
Proof. The quasilinear equation (1.8) is strictly hyperbolic with respect to the $r$-direction by $\partial_{r} \Phi>c(\rho)$. Thus, by the standard Picard iteration as in [19], one can derive that Lemma 3.1 holds.

Next, we reformulate (1.8) with (1.10)-(1.11).

Let $\dot{\Phi}=\Phi-\hat{\Phi}$. Then it follows from a direct computation that (1.8) can be reduced to:

$$
\mathcal{L} \dot{\Phi}=\dot{f} \quad \text { in } \Omega,
$$

where

$$
\left\{\begin{array}{l}
\mathcal{L} \dot{\Phi}=\partial_{r}^{2} \dot{\Phi}-\frac{P_{1}(r)}{r^{2}} \sum_{i=1}^{3} Z_{i}^{2} \dot{\Phi}+\frac{P_{2}(r)}{r} \partial_{r} \dot{\Phi}, \\
\dot{f}=f_{00} \partial_{r}^{2} \dot{\Phi}+\frac{1}{r^{2}} \sum_{i, j=1}^{3} f_{i j} Z_{i} Z_{j} \dot{\Phi}+\frac{1}{r} \sum_{i=1}^{3} f_{0 i} \partial_{r} Z_{i} \dot{\Phi}+f_{0}
\end{array}\right.
$$

with

$$
\left\{\begin{array}{l}
P_{1}(r)=\frac{c^{2}(\hat{\rho})}{\hat{U}^{2}-c^{2}(\hat{\rho})}, \\
P_{2}(r)=\frac{2}{\left(\hat{U}^{2}-c^{2}(\hat{\rho})\right)^{2}}\left((\gamma-1) \hat{U}^{4}+c^{4}(\hat{\rho})+\hat{U}^{2} c^{2}(\hat{\rho})\right)
\end{array}\right.
$$

and

$$
\left\{\begin{array}{l}
f_{00}=-\frac{1}{\hat{U}^{2}-c^{2}(\hat{\rho})}\left((\gamma+1) \hat{U} \partial_{r} \dot{\Phi}+\frac{\gamma+1}{2}\left(\partial_{r} \dot{\Phi}\right)^{2}+\frac{\gamma-1}{2 r^{2}} \sum_{i=1}^{3}\left(Z_{i} \dot{\Phi}\right)^{2}\right), \\
f_{i i}=\frac{1}{\hat{U}^{2}-c^{2}(\hat{\rho})}\left(\frac{\gamma-1}{2}\left(\partial_{r} \dot{\Phi}\right)^{2}-(\gamma-1) \hat{U} \partial_{r} \dot{\Phi}+\frac{\gamma-1}{2 r^{2}} \sum_{k=1}^{3}\left(Z_{k} \dot{\Phi}\right)^{2}-\frac{1}{r^{2}}\left(Z_{i} \dot{\Phi}\right)^{2}\right), \quad 1 \leq i \leq 3, \\
f_{i j}=f_{j i}=-\frac{1}{r^{2}\left(\hat{U}^{2}-c^{2}(\hat{\rho})\right)} Z_{i} \dot{\Phi} Z_{j} \dot{\Phi}, \quad 1 \leq i \neq j \leq 3, \\
f_{0 i}=-\frac{1}{r\left(\hat{U}^{2}-c^{2}(\hat{\rho})\right)}\left(\hat{U}+\partial_{r} \dot{\Phi}\right) Z_{i} \dot{\Phi}, \quad 1 \leq i \leq 3, \\
f_{0}=f_{0}^{1}+f_{0}^{2}, \\
f_{0}^{1}=\frac{1}{\hat{U}^{2}-c^{2}(\hat{\rho})}\left\{-\sum_{i, j=1}^{3} \frac{C_{i j}}{r^{3}} \partial_{r} \dot{\Phi} Z_{i} \dot{\Phi} Z_{j} \dot{\Phi}-\sum_{i, j, k=1}^{3} \frac{C_{i j k}}{r^{4}} Z_{i} \dot{\Phi} Z_{j} \dot{\Phi} Z_{k} \dot{\Phi}\right. \\
\left.\quad+\frac{2}{r}\left(\frac{\gamma-1}{2}\left(\partial_{r} \dot{\Phi}\right)^{3}-(\gamma-1) \hat{U}\left(\partial_{r} \dot{\Phi}\right)^{2}+\frac{\gamma-1}{2 r^{2}} \sum_{i=1}^{3}\left(Z_{i} \dot{\Phi}\right)^{2} \partial_{r} \dot{\Phi}+\frac{\gamma-1}{2} \hat{U}\left(\partial_{r} \dot{\Phi}\right)^{2}\right)\right\}, \\
f_{0}^{2}=\frac{\hat{U}}{\hat{U}^{2}-c^{2}(\hat{\rho})}\left(-\sum_{i, j=1}^{3} \frac{C_{i j}}{r^{3}} Z_{i} \dot{\Phi} Z_{j} \dot{\Phi}+\frac{\gamma-1}{r^{3}} \sum_{i=1}^{3}\left(Z_{i} \dot{\Phi}\right)^{2}\right) .
\end{array}\right.
$$


Here we point out that the terms $f_{0}^{1}=O\left(\frac{\left(\partial_{r} \dot{\Phi} Z \dot{\Phi}\right)^{2}}{r^{3}}\right)+O\left(\frac{(Z \dot{\Phi})^{3}}{r^{4}}\right)+O\left(\frac{\left(\partial_{r} \dot{\Phi}\right)^{2}}{r}\right)$ and $f_{0}^{2}=$ $O\left(\frac{(Z \dot{\Phi})^{2}}{r^{3}}\right)$ appeared in $f_{0}$ will be treated differently since only such kinds of estimates of $\left|\partial_{r} \dot{\Phi}\right| \leq C \varepsilon r^{-2(\gamma-1)} \rightarrow 0$ and $|Z \dot{\Phi}| \leq C \varepsilon r^{1-\sigma} \not \rightarrow 0$ as $r \rightarrow \infty$ are derived in $\S 5$ (one can the details in Lemma 5.4 and Lemma 5.5 below). In fact, $f_{0}^{1}$ can be easily estimated since it admits better decay rate with respect to large $r$.

On the nozzle wall $\varphi=\varphi_{0}$, $\dot{\Phi}$ satisfies

$$
Z_{2} \dot{\Phi}=Z_{3} \dot{\Phi}=0
$$

In addition, we have the following initial data of $\dot{\Phi}$ from (1.11)

$$
\dot{\Phi}(1, \varphi)=\varepsilon \Phi_{0}(\varphi), \quad \partial_{r} \dot{\Phi}(1, \varphi)=\varepsilon \Phi_{1}(\varphi),
$$

By using Lemma 2.1 and direct computations, we can obtain the following estimates on the coefficients of $\mathcal{L} \dot{\Phi}$ in (3.1):

\section{Lemma 3.1.}

$$
\begin{aligned}
P_{1}(r)= & O\left(r^{2(1-\gamma)}\right)>0, \\
P_{2}(r)= & 2(\gamma-1)+O\left(r^{2(1-\gamma)}\right)>0, \\
P_{1}^{\prime}(r)= & -\frac{c^{2}(\hat{\rho}(r))}{r\left(\hat{U}^{2}-c^{2}(\hat{\rho}(r))\right.}\left(2(\gamma-1) \hat{U}^{4}+2 \hat{U}^{2} c^{2}(\hat{\rho}(r))\right)=O\left(r^{1-2 \gamma}\right)<0, \\
\frac{P_{1}^{\prime}(r)}{P_{1}}= & O\left(r^{-1}\right)<0, \\
P_{2}^{\prime}(r)= & \frac{c^{2}(\hat{\rho}(r))}{r\left(\hat{U}^{2}-c^{2}(\hat{\rho}(r))\right)^{3}}\left(\left(12(\gamma-1) \hat{U}^{4}-8(\gamma-2) \hat{U}^{2} c^{2}(\hat{\rho}(r))\right)\left(\hat{U}^{2}-c^{2}(\hat{\rho}(r))\right)\right. \\
& \left.-2(\gamma+1) \hat{U}^{2}\left(2(\gamma-1) \hat{U}^{4}+2 \hat{U}^{2} c^{2}(\hat{\rho}(r))+2 c^{4}(\hat{\rho}(r))\right)\right)=O\left(r^{1-2 \gamma}\right) .
\end{aligned}
$$

Remark 3.1. From Lemma 3.1, if we take $r$ as the time t, then we know that the main part of $\mathcal{L}$ is like the seconder order operator $\partial_{t}^{2}-\frac{1}{(1+t)^{2(\gamma-1)}} \Delta+\frac{2(\gamma-1)}{1+t} \partial_{t}$, which is strictly hyperbolic but degenerate as $t \rightarrow \infty$. Recently, with respect to the semilinear wave equations with the forms of $\partial_{t}^{2} u-\Delta u+\frac{\mu}{(1+t)^{\alpha}} \partial_{t} u=f(u)$, where $\mu>0$ and $\alpha>0$ are suitable constants, there have been extensive and interesting works on the global existence or blowup results for the different nonlinear function $f(u)$, one can see [8], [23-24] and the references therein.

\section{$\S 4$. A first-order weighted energy estimate}

In this section, we establish a weighted energy estimate of $\nabla_{x} \dot{\Phi}$ for the linear part of (3.1) together with (3.4)-(3.5), which will play a fundamental role in our subsequent analysis. 
Set $D_{T}=\left\{(r, \theta, \varphi): 1<r<T, 0 \leq \theta \leq 2 \pi, 0 \leq \varphi<\varphi_{0}\right\}$ for any $T>1, B_{T}=$ $\left\{(r, \theta, \varphi) \in \Sigma: 1<r<T, 0 \leq \theta \leq 2 \pi, \varphi=\varphi_{0}\right\}$, and $S_{T}=\bar{\Omega} \cap\{r=T\}$.

Theorem 4.1. Let $\dot{\Phi} \in C^{2}\left(\bar{D}_{T}\right)$ satisfy the boundary condition (3.4) and initial data condition (3.5). Then there exists a multiplier $\mathcal{M} \dot{\Phi}=r^{\mu} a(r) \partial_{r} \dot{\Phi}$ such that for fixed constant $\mu=4 \gamma-6$ we have

$$
\begin{aligned}
& T^{\mu} \int_{S_{T}}\left(\partial_{r} \dot{\Phi}\right)^{2} d S+T^{\mu-2 \gamma} \int_{S_{T}}(Z \dot{\Phi})^{2} d S+C \int_{D_{T}}\left(r^{\mu-1-\delta}\left(\partial_{r} \dot{\Phi}\right)^{2}+r^{\mu-1-2 \gamma}(Z \dot{\Phi})^{2}\right) d x \\
& \leq \int_{D_{T}} \mathcal{L} \dot{\Phi} \cdot \mathcal{M} \dot{\Phi} d x+C \varepsilon^{2},
\end{aligned}
$$

where $(Z \dot{\Phi})^{2}=\sum_{k=1}^{3}\left(Z_{k} \dot{\Phi}\right)^{2}, C>0$ is a generic positive constant, and $\delta>0$ is a fixed constant.

Remark 4.1. Here we emphasize that the choice of $\mu=4 \gamma-6$ in (4.1) is very necessary due to the following two reasons: First, to guarantee the positivity of (4.3) below, one should let $\mu \leq 4 \gamma-6$; Second, by the Bernoulli's law (1.2), we have $c^{2}(\rho)=c^{2}(\hat{\rho})-\frac{\gamma-1}{2}\left(\left(\partial_{r} \dot{\Phi}\right)^{2}+\right.$ $\left.2 \hat{U} \partial_{r} \dot{\Phi}+\frac{1}{r^{2}}(Z \dot{\Phi})^{2}\right)$. Notice that only the estimate of $\left|\nabla_{x} \dot{\Phi}\right| \leq C \varepsilon r^{-\frac{\mu}{2}-1}$ can be obtained by the analysis in $\S 5-\S 6$. On the other hand, $c^{2}(\hat{\rho}(r)) \geq C r^{-2(\gamma-1)}$ and $\hat{U}=O(1)$ hold by Lemma 2.1. Therefore, in order to guarantee the absence of vacuum for any finite $r$ in $\Omega$, we require to choose the constant $\mu$ such that $-\frac{\mu}{2}-1 \leq-2(\gamma-1)$, which leads to $\mu \geq 4 \gamma-6$. Combining these two reasons yields $\mu=4 \gamma-6$.

Remark 4.2. In Theorem 4.1, it suffices to choose the constant $\delta>0$. However, to derive the higher order energy estimates of $\dot{\Phi}$, we require to give more restrictions on $\delta$ (one can see Theorem 5.1 in $\S 5$ ).

Proof. It follows from the integration by parts and (3.4)-(3.5) that

$$
\begin{aligned}
& \int_{D_{T}} \mathcal{L} \dot{\Phi} \cdot \mathcal{M} \dot{\Phi} d x \\
& =\int_{S_{T}} \frac{1}{2} r^{\mu} a(r)\left(\partial_{r} \dot{\Phi}\right)^{2} d S+\int_{S_{T}} \frac{1}{2} r^{\mu-2} P_{1} a(r)(Z \dot{\Phi})^{2} d S \\
& \quad-\left(\int_{S_{1}} \frac{1}{2} r^{\mu} a(r)\left(\partial_{r} \dot{\Phi}\right)^{2} d S+\int_{S_{1}} \frac{1}{2} r^{\mu-2} P_{1} a(r)(Z \dot{\Phi})^{2} d S\right) \\
& \quad+\int_{D_{T}}\left(r^{\mu-1}\left(\left(P_{2}-\frac{\mu+2}{2}\right) a(r)-\frac{1}{2} r a^{\prime}(r)\right)\left(\partial_{r} \dot{\Phi}\right)^{2}-\frac{1}{2} r^{\mu-3}\left(\left(\mu P_{1}+r P_{1}^{\prime}\right) a(r)+r a^{\prime}(r) P_{1}\right)(Z \dot{\Phi})^{2}\right) d x \\
& \quad+\int_{B_{T}} r^{\mu} a(r) P_{1}\left(-x_{2} Z_{2} \dot{\Phi}+x_{1} Z_{3} \dot{\Phi}\right) \partial_{r} \dot{\Phi} \\
& \geq \int_{S_{T}} \frac{1}{2} r^{\mu} a(r)\left(\partial_{r} \dot{\Phi}\right)^{2} d S+\int_{S_{T}} \frac{1}{2} r^{\mu-2} P_{1} a(r)(Z \dot{\Phi})^{2} d S \\
& \quad+\int_{D_{T}}\left(r^{\mu-1}\left(\left(P_{2}-\frac{\mu+2}{2}\right) a(r)-\frac{1}{2} r a^{\prime}(r)\right)\left(\partial_{r} \dot{\Phi}\right)^{2}-\frac{1}{2} r^{\mu-3}\left(\left(\mu P_{1}+r P_{1}^{\prime}\right) a(r)+r a^{\prime}(r) P_{1}\right)(Z \dot{\Phi})^{2}\right) d x \\
& \left.\quad-C \varepsilon^{2}\right) \quad(4.2)
\end{aligned}
$$


It is noted that

$$
\begin{aligned}
& \left(P_{2}-\frac{\mu+2}{2}\right) a(r)-\frac{1}{2} r a^{\prime}(r) \\
& =\frac{1}{2\left(\hat{U}^{2}-c^{2}(\hat{\rho})\right)}\left((4 \gamma-6-\mu) \hat{U}^{4}+(4+2(\mu+2)) \hat{U}^{2} c^{2}(\hat{\rho})+(2-\mu) c^{4}((\hat{\rho}))\right) a(r)-\frac{1}{2} r a^{\prime}(r),
\end{aligned}
$$

then in order to guarantee the positivity of (4.3) for $\mu=4 \gamma-6$, we require

$$
a(r)>0 \quad \text { and } a^{\prime}(r)<0 .
$$

For this end, we choose

$$
a(r)=1+r^{-\delta} \quad \text { with } \delta>0 .
$$

In this case, one can arrive at

$$
\left(P_{2}-\frac{\mu+2}{2}\right) a(r)-\frac{1}{2} r a^{\prime}(r)>\frac{1}{2} \delta r^{-\delta} .
$$

On the other hand, it follows from a direct computation and the assumption of $1<\gamma<2$ that

$$
\begin{aligned}
& -\left(\mu P_{1}+r \partial_{r} P_{1}\right) a(r)-r a^{\prime}(r) P_{1} \\
& =\frac{c^{2}(\hat{\rho})}{\left(\hat{U}^{2}-c^{2}(\hat{\rho})\right)^{3}}\left((2(\gamma-1)-\mu) \hat{U}^{4}+(4+2 \mu) \hat{U}^{2} c^{2}(\hat{\rho})-\mu c^{4}(\hat{\rho})\right) a(r)+\frac{\delta c^{2}(\hat{\rho}) r^{-\delta}}{\hat{U}^{2}-c^{2}(\hat{\rho})} \\
& >\frac{2 c^{2}(\hat{\rho})}{\left(\hat{U}^{2}-c^{2}(\hat{\rho})\right)^{3}}(2-\gamma) \hat{U}^{4} \\
& >C r^{-2(\gamma-1)}
\end{aligned}
$$

Thus, substituting (4.5)-(4.6) into (4.2) yields Theorem 4.1.

\section{$\S 5$. Higher-order weighted energy estimates of $\dot{\Phi}$}

In this section, we will derive the higher-order energy estimates of solution $\dot{\Phi}$ to (3.1) with (3.4)-(3.5) so that the suitable decay properties of $\nabla_{x} \dot{\Phi}$ can be obtained and the density $\rho(x)>$ 0 can be also derived in subsequent $\S 6$. Due to the Neumann boundary condition (3.4), the asymptotic degeneracy of some coefficients in (3.1), and the different decay rates of $\partial_{r} \dot{\Phi}$ and $\frac{Z \dot{\Phi}}{r}$, the related derivation procedure will become rather complicated and technical.

Theorem 5.1. Let $\dot{\Phi} \in C^{4}\left(\bar{D}_{T}\right)$ be the solution to (3.1) with (3.4)-(3.5), and further assume

$$
\sum_{0 \leq l_{1}+l_{2} \leq 1} r^{l_{1}}\left|\partial_{r}^{l_{1}} Z^{l_{2}} \partial_{r} \dot{\Phi}\right| \leq M \varepsilon r^{-2(\gamma-1)}, \quad r^{-1}|Z \dot{\Phi}| \leq M \varepsilon r^{-\sigma}, \quad r^{-1}\left|Z^{2} \dot{\Phi}\right| \leq M \varepsilon r^{-(\gamma-1)}
$$


where $M>0$ is a constant, and $\sigma=\min \{1,2(\gamma-1)\}$. Then for sufficiently small $\varepsilon>0$ and $0 \leq k \leq 3$, we have

$$
\begin{aligned}
& T^{\mu+2 k} \int_{S_{T}}\left|\nabla_{x}^{k} \partial_{r} \dot{\Phi}\right|^{2} d S+T^{\mu-2 \gamma+2 k} \int_{S_{T}}\left|\nabla_{x}^{k}\left(\frac{1}{r} Z \dot{\Phi}\right)\right|^{2} d S \\
& \quad+\int_{D_{T}}\left(r^{\mu-1-\delta+2 k}\left|\nabla_{x}^{k} \partial_{r} \dot{\Phi}\right|^{2}+r^{\mu+1-2 \gamma+2 k}\left|\nabla_{x}^{k}\left(\frac{1}{r} Z \dot{\Phi}\right)\right|^{2}\right) d x \\
& \leq C \varepsilon^{2}
\end{aligned}
$$

where $\mu=4 \gamma-6,0<\delta \leq \min \{\gamma-1, \sigma-(\gamma-1)\}$, and the domains $D_{T}, B_{T}, S_{T}$ have been defined in the beginning of $\S 4$.

In order to prove Theorem 5.1, we will apply the induction method on $k$ in (5.2) to establish the following estimates respectively:

(i) $\partial_{r} S^{k} \dot{\Phi}$ and $Z S^{k} \dot{\Phi}$ with $S=r \partial_{r}$ and $1 \leq k \leq 3$ (in this case, all the radial derivatives of $\nabla_{x} \dot{\Phi}$ up to third order are treated);

(ii) $\partial_{r} Z \dot{\Phi}$ and $Z^{2} \dot{\Phi}$ (in this case, together with the case $k=1$ in (i), all the second order derivatives $\nabla_{x}^{2} \dot{\Phi}$ are treated);

(iii) $\partial_{r} S Z \dot{\Phi}, Z S Z \dot{\Phi}, \partial_{r} Z^{2} \dot{\Phi}$ and $Z^{3} \dot{\Phi}$ (in this case, together with the case $k=2$ in (i), all the third order derivatives $\nabla_{x}^{3} \dot{\Phi}$ are treated);

(iv) $\partial_{r} S^{2} Z \dot{\Phi}, Z S^{2} Z \dot{\Phi}, \partial_{r} S Z^{2} \dot{\Phi}, Z S Z^{2} \dot{\Phi}, \partial_{r} Z^{3} \dot{\Phi}$ and $Z^{4} \dot{\Phi}$ (in this case, together with the case $k=3$ in (i), all the fourth order derivatives $\nabla_{x}^{4} \dot{\Phi}$ are treated).

These estimates will be given in Lemma 5.2-Lemma 5.5 respectively.

At first, we establish the radial derivative estimates of $\dot{\Phi}$ under the suitable induction assumption. Set $S=r \partial_{r}$, which is tangent to fixed nozzle wall $\Sigma$, then we have

Lemma 5.2. (Radial derivative estimates) Under the assumptions of Theorem 5.1, if (5.2) holds for $0 \leq l \leq m-1$ with $1 \leq m \leq 3$, then

$$
\begin{aligned}
& T^{\mu} \int_{S_{T}}\left(\partial_{r} S^{m} \dot{\Phi}\right)^{2} d S+T^{\mu-2 \gamma} \int_{S_{T}}\left(Z S^{m} \dot{\Phi}\right)^{2} d S+\int_{D_{T}}\left(r^{\mu-1-\delta}\left(\partial_{r} S^{m} \dot{\Phi}\right)^{2}+r^{\mu-1-2 \gamma}\left(Z S^{m} \dot{\Phi}\right)^{2}\right) d x \\
& \leq C \varepsilon^{2}+C \varepsilon \int_{D_{T}} \sum_{l=0}^{m}\left(r^{\mu+1-2 \gamma+2 l}\left(\nabla_{x}^{l}\left(\frac{1}{r} Z \dot{\Phi}\right)\right)^{2}+r^{\mu-1-\delta+2 l}\left(\nabla_{x}^{l} \partial_{r} \dot{\Phi}\right)^{2}\right) d x \\
& \quad+C \varepsilon \sum_{l=0}^{m}\left(T^{\mu+2 l} \int_{S_{T}}\left(\nabla_{x}^{l} \partial_{r} \dot{\Phi}\right)^{2} d S+T^{\mu-2 \gamma+2 l} \int_{S_{T}}\left(\nabla_{x}^{l}(Z \dot{\Phi})\right)^{2} d S\right) \\
& \quad+C \varepsilon\left(\int_{D_{T}} r^{\mu-1-\delta}\left(\partial_{r} S^{m} \dot{\Phi}\right)^{2}+r^{\mu-1-2 \gamma}\left(Z S^{m} \dot{\Phi}\right)^{2} d x\right)^{\frac{1}{2}}
\end{aligned}
$$

where $0<\delta \leq \gamma-1$.

Especially, for $m=0$, the following estimate holds

$$
\begin{aligned}
& T^{\mu} \int_{S_{T}}\left(\partial_{r} \dot{\Phi}\right)^{2} d S+T^{\mu-2 \gamma} \int_{S_{T}}(Z \dot{\Phi})^{2} d S+\int_{D_{T}}\left(r^{\mu-1-\delta}\left(\partial_{r} \dot{\Phi}\right)^{2}+r^{\mu-1-2 \gamma}(Z \dot{\Phi})^{2}\right) d x \\
& \leq C \varepsilon^{2}+C \varepsilon \int_{D_{T}}\left(r^{\mu+1-2 \gamma}\left(\frac{1}{r} Z \dot{\Phi}\right)^{2}+r^{\mu-1-\delta}\left(\partial_{r} \dot{\Phi}\right)^{2}\right) d x
\end{aligned}
$$




$$
+C \varepsilon\left(T^{\mu} \int_{S_{T}}\left(\partial_{r} \dot{\Phi}\right)^{2} d S+T^{\mu-2 \gamma} \int_{S_{T}}(Z \dot{\Phi})^{2} d S\right)
$$

Remark 5.1. For the case of $m=0$ in (5.4), we do not require any induction assumption.

Remark 5.2. It is noted that the angular derivatives of $\dot{\Phi}$ are still included in the right hand side of (5.3), which implies that we have not obtained the complete estimates on the radial derivative estimates of $\dot{\Phi}$. However, since the coefficients of angular derivatives of $\dot{\Phi}$ in (5.3) are small, then together with the subsequent angular derivative estimates, we can derive (5.2).

Proof. Noticing that on $\Sigma$

$$
S^{m} Z_{2} \dot{\Phi}=S^{m} Z_{3} \dot{\Phi}=0 .
$$

This, together with Theorem 4.1 and (3.5), yields

$$
\begin{aligned}
& T^{\mu} \int_{S_{T}}\left(\partial_{r} S^{m} \dot{\Phi}\right)^{2} d S+T^{\mu-2 \gamma} \int_{S_{T}}\left(Z S^{m} \dot{\Phi}\right)^{2} d S+\int_{D_{T}}\left(r^{\mu-1-\delta}\left(\partial_{r} S^{m} \dot{\Phi}\right)^{2}+r^{\mu-1-2 \gamma}\left(Z S^{m} \dot{\Phi}\right)^{2}\right) d x \\
& \leq C \int_{D_{T}} \mathcal{L} S^{m} \dot{\Phi} \cdot \mathcal{M} S^{m} \dot{\Phi} d x+C \varepsilon^{2}
\end{aligned}
$$

Next, we derive an explicit representation of $\mathcal{L} S^{m} \dot{\Phi}$ for the later uses.

By a direct computation, we have

$$
\mathcal{L} S=S \mathcal{L}+2 \mathcal{L}+\frac{P_{1}^{\prime}}{r} \sum_{i=1}^{3} Z_{i}^{2}+A_{1}
$$

where $A_{1}=-P_{2}^{\prime} \partial_{r}$ is a first order operator.

By induction, for $1 \leq m \leq 3$, we further arrive at

$$
\mathcal{L} S^{m}=S^{m} \mathcal{L}+m S^{m-1}\left(\frac{P_{1}^{\prime}}{r} \sum_{i=1}^{3} Z_{i}^{2}\right)+\sum_{0 \leq l \leq m-1} C_{l m} S^{l} \mathcal{L} \dot{\Phi}+A_{m},
$$

where $C_{l m}$ are some suitable constants, $A_{m}$ stands for a lower order differential operator whose order is less than $m$. For examples,

$$
\begin{aligned}
& A_{2}=\left(\frac{2 P_{1}^{\prime}}{r}-r\left(\frac{P_{1}^{\prime}}{r}\right)^{\prime}\right) \sum_{i=1}^{3} Z_{i}^{2}+S A_{1}+A_{1} S+A_{1}, \\
& A_{3}=S A_{2}+\left[\frac{P_{1}^{\prime}}{r} \sum_{i=1}^{3} Z_{i}^{2}, S^{2}\right]+2 S\left(\frac{P_{1}^{\prime}}{r} \sum_{i=1}^{3} Z_{i}^{2}\right)+A_{2}+A_{1} S^{2},
\end{aligned}
$$

here and below, $[\cdot, \cdot]$ denotes the usual commutator.

For convenient treatments, for $1 \leq m \leq 3$, we rewrite (5.8) as

$$
\mathcal{L} S^{m}=S^{m} \mathcal{L}+B_{1 m}+B_{2 m}
$$


with

$$
\begin{aligned}
B_{1 m} & =\sum_{0 \leq l \leq m-1} C_{l m} S^{l} \mathcal{L}+\sum_{0 \leq l \leq m-2} C_{l m} S^{m-1-l}\left(\frac{r P_{1}^{\prime}}{P_{1}}\right) S^{l}\left(\frac{P_{1}}{r^{2}} \sum_{i=1}^{3} Z_{i}^{2}\right)+A_{m}, \\
B_{2 m} & =\frac{m r P_{1}^{\prime}}{P_{1}} S^{m-1}\left(\frac{P_{1}}{r^{2}} \sum_{i=1}^{3} Z_{i}^{2}\right),
\end{aligned}
$$

where $B_{2 m} \dot{\Phi}$ contains the $(m+1)$-th order (the highest order) derivatives of $\dot{\Phi}$, but $B_{1 m} \dot{\Phi}$ only includes $\nabla_{x}^{\alpha} \dot{\Phi}$ with $|\alpha| \leq m$ (the lower order derivatives of $\dot{\Phi}$ ) and $\nabla_{x}^{m+1} \dot{\Phi}$ with small coefficients.

In addition, from the equation (3.1), for $0 \leq m \leq 3$, we have

$$
S^{m} \mathcal{L} \dot{\Phi}=I_{1}^{m}+I_{2}^{m}+I_{3}^{m},
$$

where

$$
\begin{aligned}
I_{1}^{m}= & f_{00} \partial_{r}^{2} S^{m} \dot{\Phi}+\frac{1}{r^{2}} \sum_{1 \leq i, j \leq 3} f_{i j} Z_{i} Z_{j} S^{m} \dot{\Phi}+\frac{1}{r} \sum_{i=1}^{3} f_{0 i} \partial_{r} Z_{i} S^{m} \dot{\Phi}, \\
I_{2}^{m}= & f_{00}\left[S^{m}, \partial_{r}^{2}\right] \dot{\Phi}+\frac{1}{r} \sum_{i=1}^{3} f_{0 i}\left[S^{m}, \partial_{r} Z_{i}\right] \dot{\Phi}, \\
I_{3}^{m}= & \sum_{0 \leq l \leq m} C_{l m}\left\{\sum _ { l _ { 1 } + l _ { 2 } = l , l _ { 1 } \geq 1 } \tilde { C } _ { l _ { 1 } l _ { 2 } } \left(S^{l_{1}} f_{00} S^{l_{2}} \partial_{r}^{2} \dot{\Phi}+S^{l_{1}}\left(\frac{1}{r^{2}} \sum_{1 \leq i, j \leq 3} f_{i j}\right) S^{l_{2}} Z_{i} Z_{j} \dot{\Phi}\right.\right. \\
& \left.\left.+S^{l_{1}}\left(\frac{1}{r} \sum_{i=1}^{3} f_{0 i}\right) S^{l_{2}} \partial_{r} Z_{i} \dot{\Phi}\right)\right\}+S^{m} f_{0} .
\end{aligned}
$$

Based on the preparations above, we now treat $\int_{D_{T}} \mathcal{L} S^{m} \dot{\Phi} \cdot \mathcal{M} S^{m} \dot{\Phi}$ in the right hand side of (5.6). This procedure is divided into the following five parts.

Part 1. The estimate of $\int_{D_{T}} I_{1}^{m} \cdot \mathcal{M} S^{m} \Phi d x$

Notice that we have for $C^{1}$-smooth functions $g_{i}(1 \leq i \leq 3)$

$$
\sum_{i=1}^{3} Z_{i} g_{i}=\partial_{1}\left(x_{3} g_{3}-x_{2} g_{1}\right)+\partial_{2}\left(x_{1} g_{1}-x_{3} g_{2}\right)+\partial_{3}\left(x_{2} g_{2}-x_{1} g_{3}\right)
$$

and

$$
\int_{D_{T}} \sum_{i=1}^{3} Z_{i} g_{i} d x=-\int_{B_{T}} \frac{1}{\sin \varphi_{0}}\left(x_{2} g_{2}-x_{1} g_{3}\right) d S .
$$

In addition, a direct computation yields for $m \leq 3$

$I_{1}^{m} \cdot \mathcal{M} S^{m} \dot{\Phi}$ 


$$
\begin{aligned}
= & \partial_{r}\left(\frac{1}{2} r^{\mu} a(r) f_{00}\left(\partial_{r} S^{m} \dot{\Phi}\right)^{2}-r^{\mu-2} a(r) \sum_{1 \leq i<j \leq 3} f_{i j} Z_{i} S^{m} \dot{\Phi} Z_{j} S^{m} \dot{\Phi}-\frac{1}{2} r^{\mu-2} a(r) \sum_{i=1}^{3} f_{i i}\left(Z_{i} S^{m} \dot{\Phi}\right)^{2}\right) \\
& +\sum_{i=1}^{3} Z_{i}\left(\frac{1}{2} r^{\mu-1} a(r) f_{0 i}\left(\partial_{r} S^{m} \dot{\Phi}\right)^{2}+r^{\mu-2} a(r) \partial_{r} S^{m} \dot{\Phi} \sum_{j=1}^{3} f_{i j} Z_{j} S^{m} \dot{\Phi}\right) \\
& -\frac{1}{2} \partial_{r}\left(r^{\mu} a(r) f_{00}\right)\left(\partial_{r} S^{m} \dot{\Phi}\right)^{2}-\frac{1}{2} r^{\mu-1} a(r)\left(\partial_{r} S^{m} \dot{\Phi}\right)^{2} \sum_{i=1}^{3} Z_{i} f_{0 i}+\sum_{i=1}^{3} \partial_{r}\left(\frac{1}{2} r^{\mu-2} a(r) f_{i i}\right)\left(Z_{i} S^{m} \dot{\Phi}\right)^{2} \\
& +\sum_{1 \leq i<j \leq 3} \partial_{r}\left(r^{\mu-2} a(r) f_{i j}\right) Z_{i} S^{m} \dot{\Phi} Z_{j} S^{m} \dot{\Phi}
\end{aligned}
$$

On the other hand, by the expressions of $f_{i j}, f_{0 i}$ and (5.5), a crucial observation yields on $B_{T}$

$$
\begin{aligned}
x_{2}( & \left.\frac{1}{2} r^{\mu-1} a(r) f_{02}\left(\partial_{r} S^{m} \dot{\Phi}\right)^{2}+r^{\mu-2} a(r) \partial_{r} S^{m} \dot{\Phi} \sum_{j=1}^{3} f_{2 j} Z_{j} S^{m} \dot{\Phi}\right) \\
& -x_{1}\left(\frac{1}{2} r^{\mu-1} a(r) f_{03}\left(\partial_{r} S^{m} \dot{\Phi}\right)^{2}+r^{\mu-2} a(r) \partial_{r} S^{m} \dot{\Phi} \sum_{j=1}^{3} f_{3 j} Z_{j} S^{m} \dot{\Phi}\right) \\
= & \frac{1}{2} a(r)\left(\partial_{r} S^{m} \dot{\Phi}\right)^{2}\left(x_{2} f_{02}-x_{1} f_{03}\right)+r^{\mu-2} a(r) \partial_{r} S^{m} \dot{\Phi} \sum_{j=1}^{3}\left(x_{2} f_{2 j}-x_{1} f_{3 j}\right) Z_{j} S^{m} \dot{\Phi} \\
= & \frac{1}{\hat{U}^{2}-c^{2}(\hat{\rho})} r^{\mu-2} a(r) \partial_{r} S^{m} \dot{\Phi}\left(\frac{\gamma-1}{2}\left(\partial_{r} \dot{\Phi}\right)^{2}-(\gamma-1) \hat{U} \partial_{r} \dot{\Phi}+\frac{\gamma-1}{2 r^{2}} \sum_{k=1}^{3}\left(Z_{k} \dot{\Phi}\right)^{2}\right)\left(x_{2} Z_{2} S^{m} \dot{\Phi}\right. \\
& \left.-x_{1} Z_{3} S^{m} \dot{\Phi}\right)-\frac{1}{\hat{U}^{2}-c^{2}(\hat{\rho})} r^{\mu-4} a(r) \partial_{r} S^{m} \dot{\Phi} \sum_{j=1}^{3} Z_{j} \dot{\Phi} Z_{j} S^{m} \dot{\Phi}\left(x_{2} Z_{2} \dot{\Phi}-x_{1} Z_{3} \dot{\Phi}\right) \\
& -\frac{1}{2} r^{\mu-2} a(r)\left(\hat{U}+\partial_{r} \dot{\Phi}\right)\left(\partial_{r} S^{m} \dot{\Phi}\right)^{2}\left(x_{2} Z_{2} \dot{\Phi}-x_{1} Z_{3} \dot{\Phi}\right) \\
= & 0 .
\end{aligned}
$$

Thus, by (5.13) together with (5.12) and (5.14), it follows from an integration by parts and simultaneously notices the expressions of $f_{i}$ and the assumption (5.1) that

$$
\begin{aligned}
& \left|\int_{D_{T}} I_{1}^{m} \cdot \mathcal{M} S^{m} \dot{\Phi} d x\right| \\
& \leq C \varepsilon^{2}+C \varepsilon\left(T^{\mu} \int_{S_{T}}\left(\partial_{r} S^{m} \dot{\Phi}\right)^{2} d S+T^{\mu-2 \gamma} \int_{S_{T}}\left(Z S^{m} \dot{\Phi}\right)^{2} d S\right. \\
& \left.\quad+\int_{D_{T}}\left(r^{\mu-1-\delta}\left(\partial_{r} S^{m} \dot{\Phi}\right)^{2}+r^{\mu-1-2 \gamma}\left(Z S^{m} \dot{\Phi}\right)^{2}\right) d x\right)
\end{aligned}
$$


here we have used some facts such as

$\left|r^{\mu-1} a(r)\left(\partial_{r} S^{m} \dot{\Phi}\right)^{2} \sum_{i=1}^{3} Z_{i} f_{0 i}\right| \leq C \varepsilon r^{\mu-1-(\gamma-1)}\left(\partial_{r} S^{m} \dot{\Phi}\right)^{2} \leq C \varepsilon r^{\mu-1-\delta}\left(\partial_{r} S^{m} \dot{\Phi}\right)^{2} \quad$ for $0<\delta \leq \gamma-1$.

Part 2. The estimate of $\int_{D_{T}} I_{2}^{m} \cdot \mathcal{M} S^{m} \Phi d x$

It follows from the expressions of $f_{i}$, Lemma 2.5 (ii) and (5.1) that

$$
\left|I_{2}^{m}\right| \leq C \varepsilon\left(r^{-\sigma-1} \sum_{0 \leq l \leq m-1}\left|S^{l} \partial_{r} Z \dot{\Phi}\right|+r^{-2(\gamma-1)} \sum_{0 \leq l \leq m-1}\left|S^{l} \partial_{r}^{2} \dot{\Phi}\right|\right),
$$

which derives that

$$
\int_{D_{T}}\left|I_{2}^{m} \cdot \mathcal{M} S^{m} \dot{\Phi}\right| d x \leq C \varepsilon \int_{D_{T}}\left(\sum_{0 \leq l \leq m-1} r^{\mu-1-\delta}\left(S^{l} \partial_{r} Z \dot{\Phi}\right)^{2}+\sum_{1 \leq l \leq m} r^{\mu-1-\delta}\left(S^{l} \partial_{r} \dot{\Phi}\right)^{2}\right) d x .
$$

Part 3. The estimate of $\int_{D_{T}} I_{3}^{m} \cdot \mathcal{M} S^{m} \Phi d x$

At first, we treat the case of $\int_{D_{T}} I_{3}^{m} \cdot \mathcal{M} S^{m} \Phi d x$ with $m \leq 2$.

For $m \leq 2$, as in Part 2 it follows from the expressions of $f_{i}$ and the assumption (5.1) that

$$
\left|I_{3}^{m}\right| \leq C \varepsilon\left(\sum_{0 \leq l \leq m}\left(r^{-\gamma}\left|S^{l} \partial_{r} \dot{\Phi}\right|+r^{-\sigma-2}\left|S^{l} Z \dot{\Phi}\right|\right)+\sum_{0 \leq l \leq m-1} r^{-2 \gamma}\left|S^{l} Z^{2} \dot{\Phi}\right|\right),
$$

which derives for $m \leq 2$

$$
\int_{D_{T}}\left|I_{3}^{m} \cdot \mathcal{M} S^{m} \dot{\Phi}\right| d x \leq C \varepsilon \int_{D_{T}} \sum_{l=0}^{m}\left(r^{\mu+1-2 \gamma+2 l}\left(\nabla_{x}^{l}\left(\frac{1}{r} Z \dot{\Phi}\right)\right)^{2}+r^{\mu-1-\delta+2 l}\left(\nabla_{x}^{l} \partial_{r} \dot{\Phi}\right)^{2}\right) d x .
$$

Next we deal with $\int_{D_{T}} I_{3}^{3} \cdot \mathcal{M} S^{3} \dot{\Phi} d x$.

It is noted that the most troublesome terms in $I_{3}^{3}$ are the ones which include the products of third order derivatives of $\dot{\Phi}$ since there are no related weighted $L^{\infty}$ estimates in (5.1). For the convenient treatments, we decompose $I_{3}^{3}$ into $J_{1}$ and $J_{2}$ by using $S^{2}=r \partial_{r}+r^{2} \partial_{r}^{2}$, where only $J_{2}$ contains the product terms of third order derivatives of $\dot{\Phi}$. Namely,

$$
I_{3}^{3}=J_{1}+J_{2}
$$

with

$$
\begin{aligned}
& J_{1}= \\
& \sum_{0 \leq l \leq 2} C_{l 2} \sum_{l_{1}+l_{2}=l, l_{1} \geq 1} \tilde{C}_{l_{1} l_{2}}\left(S^{l_{1}} f_{00} S^{l_{2}} \partial_{r}^{2} \dot{\Phi}+S^{l_{1}}\left(\frac{1}{r^{2}} \sum_{1 \leq i, j \leq 3} f_{i j}\right) S^{l_{2}} Z_{i} Z_{j} \dot{\Phi}+S^{l_{1}}\left(\frac{1}{r} \sum_{i=1}^{3} f_{0 i}\right) S^{l_{2}} \partial_{r} Z_{i} \dot{\Phi}\right)
\end{aligned}
$$




$$
\begin{aligned}
& +C_{33} \sum_{\left(l_{1}, l_{2}\right) \neq(2,1)} \tilde{C}_{l_{1} l_{2}}\left(S^{l_{1}} f_{00} S^{l_{2}} \partial_{r}^{2} \dot{\Phi}+S^{l_{1}}\left(\frac{1}{r^{2}} \sum_{1 \leq i, j \leq 3} f_{i j}\right) S^{l_{2}} Z_{i} Z_{j} \dot{\Phi}+S^{l_{1}}\left(\frac{1}{r} \sum_{i=1}^{3} f_{0 i}\right) S^{l_{2}} \partial_{r} Z_{i} \dot{\Phi}\right) \\
& +C_{33} \tilde{C}_{21}\left(r \partial_{r} f_{00} S \partial_{r}^{2} \dot{\Phi}+r \partial_{r}\left(\frac{1}{r^{2}} \sum_{i, j=1}^{3} f_{i j}\right) S Z_{i} Z_{j} \dot{\Phi}+r \partial_{r}\left(\frac{1}{r} \sum_{i=1}^{3} f_{0 i}\right) S \partial_{r} Z_{i} \dot{\Phi}\right)+S^{3} f_{0}
\end{aligned}
$$

and

$$
J_{2}=C_{33} \tilde{C}_{21}\left(r^{2} \partial_{r}^{2} f_{00} S \partial_{r}^{2} \dot{\Phi}+r^{2} \partial_{r}^{2}\left(\frac{1}{r^{2}} \sum_{i, j=1}^{3} f_{i j}\right) S Z_{i} Z_{j} \dot{\Phi}+r^{2} \partial_{r}^{2}\left(\frac{1}{r} \sum_{i=1}^{3} f_{0 i}\right) S \partial_{r} Z_{i} \dot{\Phi}\right) .
$$

By the assumption (5.1) and the expressions of $f_{i j}, f_{0}$, then a direct computation yields

$$
\left|J_{1}\right| \leq C \varepsilon\left\{\sum_{0 \leq l \leq 3}\left(r^{-\gamma}\left|S^{l} \partial_{r} \dot{\Phi}\right|+r^{-\sigma-2}\left|S^{l} Z \dot{\Phi}\right|\right)+\sum_{0 \leq l \leq 2} r^{-2 \gamma}\left|S^{l} Z^{2} \dot{\Phi}\right|\right\} .
$$

Next, by the expressions of $f_{i j}$ we continue to decompose $J_{2}$ as $J_{2}=J_{21}+J_{22}$ so that only $J_{22}$ contains the product terms of third order derivatives of $\dot{\Phi}$. More concretely,

$$
J_{2}=J_{21}+J_{22}
$$

with

$$
\begin{aligned}
J_{22} & =-\frac{r^{2}}{\hat{U}^{2}-c^{2}(\hat{\rho})}\left\{\left((\gamma+1)\left(\hat{U}+\partial_{r} \dot{\Phi}\right) \partial_{r}^{3} \dot{\Phi}+\frac{\gamma-1}{r^{2}} \sum_{i=1}^{3} Z_{i} \dot{\Phi} \partial_{r}^{2} Z_{i} \dot{\Phi}\right) S \partial_{r}^{2} \dot{\Phi}\right. \\
& -\frac{\gamma-1}{r^{2}} \sum_{i=1}^{3}\left(\partial_{r} \dot{\Phi} \partial_{r}^{3} \dot{\Phi}-\hat{U} \partial_{r}^{3} \dot{\Phi}+\frac{1}{r^{2}} \sum_{k=1}^{3} Z_{k} \dot{\Phi} \partial_{r}^{2} Z_{k} \dot{\Phi}-\frac{1}{r^{2}} Z_{i} \dot{\Phi} \partial_{r}^{2} Z_{i} \dot{\Phi}\right) S Z_{i}^{2} \dot{\Phi} \\
& +\frac{1}{r^{4}} \sum_{1 \leq i \neq j \leq 3}\left(Z_{i} \dot{\Phi} \partial_{r}^{2} Z_{j} \dot{\Phi}+Z_{j} \dot{\Phi} \partial_{r}^{2} Z_{i} \dot{\Phi}\right) S Z_{i} Z_{j} \dot{\Phi}+\frac{1}{r^{2}} \sum_{i=1}^{3}\left(\hat{U} \partial_{r}^{2} Z_{i} \dot{\Phi}+\partial_{r}^{3} \dot{\Phi} Z_{i} \dot{\Phi}\right. \\
& \left.\left.+\partial_{r} \dot{\Phi} \partial_{r}^{2} Z_{i} \dot{\Phi}\right) S \partial_{r} Z_{i} \dot{\Phi}\right\}
\end{aligned}
$$

and

$$
\left|J_{21}\right| \leq C \varepsilon\left(r^{-(\gamma-1)}\left|S \partial_{r}^{2} \dot{\Phi}\right|+r^{-\sigma-1}\left|S \partial_{r} Z \dot{\Phi}\right|+r^{-2 \gamma}\left|S Z^{2} \dot{\Phi}\right|\right),
$$

here we point out that (5.20) is derived by direct but tedious computations through applying Lemma 2.1, assumption (5.1) and the concrete expression of $J_{21}$.

Combining (5.19) and (5.20) together with Lemma 2.5 can yield

$$
\int_{D_{T}}\left|\left(J_{1}+J_{21}\right) \cdot \mathcal{M} S^{3} \dot{\Phi}\right| d x \leq C \varepsilon \int_{D_{T}} \sum_{l=0}^{3}\left(r^{\mu+1-2 \gamma+2 l}\left|\nabla_{x}^{l}\left(\frac{1}{r} Z \dot{\Phi}\right)\right|^{2}+r^{\mu-1-\delta+2 l}\left|\nabla_{x}^{l} \partial_{r} \dot{\Phi}\right|^{2}\right) d x .
$$


Finally we treat $\int_{D_{T}}\left|J_{22} \cdot \mathcal{M} S^{3} \dot{\Phi}\right| d x$.

To overcome the difficulties induced by the lack of weighted $L^{\infty}$ estimates of $\left|\nabla_{x}^{3} \dot{\Phi}\right|$ in $J_{22}$, we will use the interpolation inequalities in Corollary 2.3 and Lemma 2.6. In fact, by (5.1) and the expression of $J_{22}$, it is only enough to deal with the following typical terms in $\int_{D_{T}}\left|J_{22} \cdot \mathcal{M} S^{3} \dot{\Phi}\right| d x$ :

(A) Estimate of $\left|r^{\mu+2} \partial_{r}^{3} \dot{\Phi} S \partial_{r}^{2} \dot{\Phi} \partial_{r} S^{3} \dot{\Phi}\right|_{L^{1}\left(D_{T}\right)}$

$$
\begin{aligned}
& \left|r^{\mu+2} \partial_{r}^{3} \dot{\Phi} S \partial_{r}^{2} \dot{\Phi} \partial_{r} S^{3} \dot{\Phi}\right|_{L^{1}}=\left|r^{\mu+3}\left(\partial_{r}^{3} \dot{\Phi}\right)^{2} \partial_{r} S^{3} \dot{\Phi}\right|_{L^{1}} \\
= & \left|r^{\delta-2(\gamma-1)} \cdot\left(r^{\frac{8 \gamma-3-\delta}{4}} \partial_{r}^{3} \dot{\Phi}\right)^{2} \cdot r^{\frac{\mu-1-\delta}{2}} \partial_{r} S^{3} \dot{\Phi}\right|_{L^{1}} \\
\leq & \left|r^{\frac{8 \gamma-3-\delta}{4}} \partial_{r}^{3} \dot{\Phi}\right|_{L^{4}}^{2}\left|r^{\frac{\mu-1-\delta}{2}} \partial_{r} S^{3} \dot{\Phi}\right|_{L^{2}} \\
\leq & C \varepsilon \sum_{l=0}^{3}\left(\left|r^{\frac{\mu-1-\delta+2 l}{2}} \nabla_{x}^{l} \partial_{r} \dot{\Phi}\right|_{L^{2}}+\left|r^{\frac{\mu+1-2 \gamma+2 l}{2}} \nabla_{x}^{l}\left(\frac{1}{r} Z \dot{\Phi}\right)\right|_{L^{2}}\right)\left|r^{\frac{\mu-1-\delta}{2}} \partial_{r} S^{3} \dot{\Phi}\right|_{L^{2}}
\end{aligned}
$$

(Applying Lemma 2.7 for $\dot{\Phi}$ )

$$
\leq C \varepsilon \sum_{l=0}^{3}\left(\left|r^{\frac{\mu-1-\delta+2 l}{2}} \nabla_{x}^{l} \partial_{r} \dot{\Phi}\right|_{L^{2}}^{2}+\left|r^{\frac{\mu+1-2 \gamma+2 l}{2}} \nabla_{x}^{l}\left(\frac{1}{r} Z \dot{\Phi}\right)\right|_{L^{2}}^{2}\right) \text {. }
$$

(B) Estimate of $\left|r^{\mu} Z \dot{\Phi} \partial_{r}^{2} Z \dot{\Phi} S \partial_{r}^{2} \dot{\Phi} \partial_{r} S^{3} \dot{\Phi}\right|_{L^{1}\left(D_{T}\right)}+\left|r^{\mu} Z \dot{\Phi} \partial_{r}^{3} \dot{\Phi} S \partial_{r} Z \dot{\Phi} \partial_{r} S^{3} \dot{\Phi}\right|_{L^{1}\left(D_{T}\right)}$

$$
\left|r^{\mu} Z \dot{\Phi} \partial_{r}^{2} Z \dot{\Phi} S \partial_{r}^{2} \dot{\Phi} \partial_{r} S^{3} \dot{\Phi}\right|_{L^{1}\left(D_{T}\right)}+\left|r^{\mu} Z \dot{\Phi} \partial_{r}^{3} \dot{\Phi} S \partial_{r} Z \dot{\Phi} \partial_{r} S^{3} \dot{\Phi}\right|_{L^{1}\left(D_{T}\right)}
$$$$
=2\left|r^{\mu+1} Z \dot{\Phi} \partial_{r}^{3} \dot{\Phi} \partial_{r}^{2} Z \dot{\Phi} \partial_{r} S^{3} \dot{\Phi}\right|_{L^{1}\left(D_{T}\right)}
$$$$
\leq C\left|r^{\mu+2-\sigma} \partial_{r}^{3} \dot{\Phi} \partial_{r}^{2} Z \dot{\Phi} \partial_{r} S^{3} \dot{\Phi}\right|_{L^{1}\left(D_{T}\right)} \quad \text { (By assumption (5.1)) }
$$$$
=\left|r^{\delta-2(\gamma-1)-\sigma} \cdot r^{\frac{8 \gamma-3-\delta}{4}} \partial_{r}^{3} \dot{\Phi} \cdot r^{\frac{8 \gamma-7-\delta}{4}} \partial_{r}^{2} Z \dot{\Phi} \cdot r^{\frac{\mu-1-\delta}{2}} \partial_{r} S^{3} \dot{\Phi}\right|_{L^{1}\left(D_{T}\right)}
$$$$
\leq C\left|r^{\frac{8 \gamma-3-\delta}{4}} \partial_{r}^{3} \dot{\Phi}\right|_{L^{4}\left(D_{T}\right)}\left|r^{\frac{8 \gamma-7-\delta}{4}} \partial_{r}^{2} Z \dot{\Phi}\right|_{L^{4}\left(D_{T}\right)}\left|r^{\frac{\mu-1-\delta}{2}} \partial_{r} S^{3} \dot{\Phi}\right|_{L^{2}\left(D_{T}\right)}
$$$$
\leq C \varepsilon \sum_{l=0}^{3}\left(\left|r^{\frac{\mu-1-\delta+2 l}{2}} \nabla_{x}^{l} \partial_{r} \dot{\Phi}\right|_{L^{2}\left(D_{T}\right)}^{2}+\left|r^{\frac{\mu+1-2 \gamma+2 l}{2}} \nabla_{x}^{l}\left(\frac{1}{r} Z \dot{\Phi}\right)\right|_{L^{2}\left(D_{T}\right)}^{2}\right) \text {. (Applying Lemma } 2.7 \text { for } \dot{\Phi} \text { ) }
$$

(C) Estimate of $\left|r^{\mu} \partial_{r}^{3} \dot{\Phi} S Z^{2} \dot{\Phi} \partial_{r} S^{3} \dot{\Phi}\right|_{L^{1}\left(D_{T}\right)}$

$$
\begin{aligned}
& \left|r^{\mu} \partial_{r}^{3} \dot{\Phi} S Z^{2} \dot{\Phi} \partial_{r} S^{3} \dot{\Phi}\right|_{L^{1}\left(D_{T}\right)}=\left|r^{\mu+1} \partial_{r}^{3} \dot{\Phi} \partial_{r} Z^{2} \dot{\Phi} \partial_{r} S^{3} \dot{\Phi}\right|_{L^{1}\left(D_{T}\right)} \\
= & \left|r^{\delta-2(\gamma-1)} \cdot r^{\frac{8 \gamma-3-\delta}{4}} \partial_{r}^{3} \dot{\Phi} \cdot r^{\frac{8 \gamma-11-\delta}{4}} \partial_{r} Z^{2} \dot{\Phi} \cdot r^{\frac{\mu-1-\delta}{2}} \partial_{r} S^{3} \dot{\Phi}\right|_{L^{1}\left(D_{T}\right)} \\
\leq & C\left|r^{\frac{8 \gamma-3-\delta}{4}} \partial_{r}^{3} \dot{\Phi}\right|_{L^{4}\left(D_{T}\right)}\left|r^{\frac{8 \gamma-11-\delta}{4}} \partial_{r} Z^{2} \dot{\Phi}\right|_{L^{4}\left(D_{T}\right)}\left|r^{\frac{\mu-1-\delta}{2}} \partial_{r} S^{3} \dot{\Phi}\right|_{L^{2}\left(D_{T}\right)} \\
\leq & C \varepsilon \sum_{l=0}^{3}\left(\left|r^{\frac{\mu-1-\delta+2 l}{2}} \nabla_{x}^{l} \partial_{r} \dot{\Phi}\right|_{L^{2}\left(D_{T}\right)}^{2}+\left|r^{\frac{\mu+1-2 \gamma+2 l}{2}} \nabla_{x}^{l}\left(\frac{1}{r} Z \dot{\Phi}\right)\right|_{L^{2}\left(D_{T}\right)}^{2}\right)
\end{aligned}
$$

(Applying Lemma 2.7 for $\dot{\Phi}$ ) 
(D) Estimate of $\left|r^{\mu-2} Z \dot{\Phi} \partial_{r}^{2} Z \dot{\Phi} S Z^{2} \dot{\Phi} \partial_{r} S^{3} \dot{\Phi}\right|_{L^{1}\left(D_{T}\right)}$

$$
\begin{aligned}
& \left|r^{\mu-2} Z \dot{\Phi} \partial_{r}^{2} Z \dot{\Phi} S Z^{2} \dot{\Phi} \partial_{r} S^{3} \dot{\Phi}\right|_{L^{1}\left(D_{T}\right)} \\
\leq & C\left|r^{\mu-\sigma} \partial_{r}^{2} Z \dot{\Phi} \partial_{r} Z^{2} \dot{\Phi} \partial_{r} S^{3} \dot{\Phi}\right|_{L^{1}\left(D_{T}\right)} \quad \text { (By assumption (5.1)) } \\
= & C\left|r^{\delta-2(\gamma-1)-\sigma} \cdot r^{\frac{8 \gamma-7-\delta}{4}} \partial_{r}^{2} Z \dot{\Phi} \cdot r^{\frac{8 \gamma-11-\delta}{4}} \partial_{r} Z^{2} \dot{\Phi} \cdot r^{\frac{\mu-1-\delta}{2}} \partial_{r} S^{3} \dot{\Phi}\right|_{L^{1}\left(D_{T}\right)} \\
\leq & C\left|r^{\frac{8 \gamma-7-\delta}{4}} \partial_{r}^{2} Z \dot{\Phi}\right|_{L^{4}\left(D_{T}\right)}\left|r^{\frac{8 \gamma-11-\delta}{4}} \partial_{r} Z^{2} \dot{\Phi}\right|_{L^{4}\left(D_{T}\right)}\left|r^{\frac{\mu-1-\delta}{2}} \partial_{r} S^{3} \dot{\Phi}\right|_{L^{2}\left(D_{T}\right)} \\
\leq & \left.C \varepsilon \sum_{l=0}^{3}\left(\left|r^{\frac{\mu-1-\delta+2 l}{2}} \nabla_{x}^{l} \partial_{r} \dot{\Phi}\right|_{L^{2}\left(D_{T}\right)}^{2}+\left|r^{\frac{\mu+1-2 \gamma+2 l}{2}} \nabla_{x}^{l}\left(\frac{1}{r} Z \dot{\Phi}\right)\right|_{L^{2}\left(D_{T}\right)}^{2}\right) . \quad \text { (Applying Lemma 2.7 for } \dot{\Phi}\right)
\end{aligned}
$$

(E) Estimate of $\left|r^{\mu} \partial_{r}^{2} Z \dot{\Phi} S \partial_{r} Z \dot{\Phi} \partial_{r} S^{3} \dot{\Phi}\right|_{L^{1}\left(D_{T}\right)}$

$$
\begin{aligned}
& \left|r^{\mu} \partial_{r}^{2} Z \dot{\Phi} S \partial_{r} Z \dot{\Phi} \partial_{r} S^{3} \dot{\Phi}\right|_{L^{1}\left(D_{T}\right)} \\
= & \left|r^{\mu+1}\left(\partial_{r}^{2} Z \dot{\Phi}\right)^{2} \partial_{r} S^{3} \dot{\Phi}\right|_{L^{1}\left(D_{T}\right)} \\
= & \left|r^{\delta-2(\gamma-1)} \cdot\left(r^{\frac{8 \gamma-7-\delta}{4}} \partial_{r}^{2} Z \dot{\Phi}\right)^{2} \cdot r^{\frac{\mu-1-\delta}{2}} \partial_{r} S^{3} \dot{\Phi}\right|_{L^{1}\left(D_{T}\right)} \\
\leq & C\left|r^{\frac{8 \gamma-7-\delta}{4}} \partial_{r}^{2} Z \dot{\Phi}\right|_{L^{4}\left(D_{T}\right)}^{2}\left|r^{\frac{\mu-1-\delta}{2}} \partial_{r} S^{3} \dot{\Phi}\right|_{L^{2}\left(D_{T}\right)} \\
\leq & C \varepsilon \sum_{l=0}^{3}\left(\left|r^{\frac{\mu-1-\delta+2 l}{2}} \nabla_{x}^{l} \partial_{r} \dot{\Phi}\right|_{L^{2}\left(D_{T}\right)}^{2}+\left|r^{\frac{\mu+1-2 \gamma+2 l}{2}} \nabla_{x}^{l}\left(\frac{1}{r} Z \dot{\Phi}\right)\right|_{L^{2}\left(D_{T}\right)}^{2}\right) .
\end{aligned}
$$

Collecting (5.22)-(5.26), one has

$$
\int_{D_{T}}\left|J_{22} \cdot \mathcal{M} S^{3} \dot{\Phi}\right| d x \leq C \varepsilon \int_{D_{T}} \sum_{l=0}^{3}\left(r^{\mu+1-2 \gamma+2 l}\left(\nabla_{x}^{l}\left(\frac{1}{r} Z \dot{\Phi}\right)\right)^{2}+r^{\mu-1-\delta+2 l}\left(\nabla_{x}^{l} \partial_{r} \dot{\Phi}\right)^{2}\right) d x
$$

This, together with (5.17) and (5.21), yields for $m \leq 3$

$$
\int_{D_{T}}\left|I_{3}^{m} \cdot \mathcal{M} S^{m} \dot{\Phi}\right| d x \leq C \varepsilon \int_{D_{T}} \sum_{l=0}^{m}\left(r^{\mu+1-2 \gamma+2 l}\left(\nabla_{x}^{l}\left(\frac{1}{r} Z \dot{\Phi}\right)\right)^{2}+r^{\mu-1-\delta+2 l}\left(\nabla_{x}^{l} \partial_{r} \dot{\Phi}\right)^{2}\right) d x
$$

Part 4. The estimate of $\int_{D_{T}} B_{1 m} \dot{\Phi} \cdot \mathcal{M} S^{m} \Phi d x$

At first, from the expressions of $A_{k}$ with $1 \leq k \leq m$ in (5.8) and Lemma 2.1, we have

$$
\left\{\begin{array}{l}
\left|A_{1} \dot{\Phi}\right| \leq C r^{1-2 \gamma}\left|\partial_{r} \dot{\Phi}\right|, \\
\left|A_{k} \dot{\Phi}\right| \leq C r^{1-2 \gamma}\left(\sum_{l=0}^{k-1}\left|\partial_{r} S^{l} \dot{\Phi}\right|+\sum_{l=0}^{k-2}\left|\frac{1}{r} S^{l} Z^{2} \dot{\Phi}\right|\right), \quad k=2,3 .
\end{array}\right.
$$


Substituting (5.29) into $B_{1 m} \dot{\Phi}$ and using the expression of $\dot{f}$, then we have by (5.1) and a direct computation

$$
\begin{aligned}
\left|B_{11} \dot{\Phi}\right| & \leq C \varepsilon\left(\sum_{0 \leq l \leq 1}\left(r^{-\gamma}\left|S^{l} \partial_{r} \dot{\Phi}\right|+r^{-\sigma-2}\left|S^{l} Z \dot{\Phi}\right|\right)+r^{-2 \gamma}\left|Z^{2} \dot{\Phi}\right|\right)+C r^{1-2 \gamma}\left|\partial_{r} \dot{\Phi}\right|, \\
\left|B_{1 k} \dot{\Phi}\right| & \leq C \varepsilon\left(\sum_{0 \leq l \leq k}\left(r^{-\gamma}\left|S^{l} \partial_{r} \dot{\Phi}\right|+r^{-\sigma-2}\left|S^{l} Z \dot{\Phi}\right|\right)+\sum_{0 \leq l \leq k-1} r^{-2 \gamma}\left|S^{l} Z^{2} \dot{\Phi}\right|\right) \\
& +C r^{1-2 \gamma}\left(\sum_{l=0}^{k-1}\left|\partial_{r} S^{l} \dot{\Phi}\right|+\sum_{l=0}^{k-2}\left|\frac{1}{r} S^{l} Z^{2} \dot{\Phi}\right|\right), \quad k=2,3 .
\end{aligned}
$$

Since (5.2) holds for $l \leq m-1$, we then have from (5.30)-(5.31)

$$
\begin{aligned}
\int_{D_{T}} & B_{1 m} \dot{\Phi} \cdot \mathcal{M} S^{m} \Phi d x \mid \leq C \varepsilon \int_{D_{T}} \sum_{l=0}^{m}\left(r^{\mu+1-2 \gamma+2 l}\left(\nabla_{x}\left(\frac{Z \dot{\Phi}}{r}\right)\right)^{2}+r^{\mu-1-\delta+2 l}\left(\nabla_{x}^{l} \partial_{r} \dot{\Phi}\right)^{2}\right) d x \\
& +C \varepsilon\left(\int_{D_{T}} r^{\mu-1-\delta}\left(\partial_{r} S^{m} \dot{\Phi}\right)^{2} d x\right)^{\frac{1}{2}} .
\end{aligned}
$$

Part 5. The estimate of $\int_{D_{T}} B_{2 m} \dot{\Phi} \cdot \mathcal{M} S^{m} \Phi d x$

It is noted that $B_{2 m} \dot{\Phi}$ contains the $(m+1)$-th (the highest order) derivatives of $\dot{\Phi}$ and then $B_{2 m} \dot{\Phi} \cdot \mathcal{M} S^{m} \Phi$ will contain the term $\nabla_{x}^{\alpha} \dot{\Phi} \nabla_{x}^{\beta} \dot{\Phi}(|\alpha|=|\beta|=m+1)$ which will yield serious troubles in the general case. However, thanks to $\frac{P_{1}^{\prime}(r)}{P_{1}(r)}<0$ given in Lemma 3.1 and the good form of (3.1), the bad influence of $\nabla_{x}^{\alpha} \dot{\Phi} \nabla_{x}^{\beta} \dot{\Phi}$ with $|\alpha|=|\beta|=m+1$ can be eliminated in the related energy estimates. We now give the details.

Since

$$
\partial_{r} S^{m} \dot{\Phi}=\sum_{0 \leq l \leq m-1} C_{l m} \partial_{r} S^{l} \dot{\Phi}+r S^{m-1} \partial_{r}^{2} \dot{\Phi}
$$

then it follows from (3.1) that

$$
\partial_{r} S^{m} \dot{\Phi}=\sum_{0 \leq l \leq m-1} C_{l m} \partial_{r} S^{l} \dot{\Phi}+r S^{m-1}\left(\frac{1}{r^{2}} P_{1} \sum_{i=1}^{3} Z_{i}^{2} \dot{\Phi}+\mathcal{L} \dot{\Phi}-\frac{1}{r} P_{2} \partial_{r} \dot{\Phi}\right) .
$$

A direct computation yields

$$
\begin{aligned}
& \int_{D_{T}} B_{2 m} \dot{\Phi} \cdot \mathcal{M} S^{m} \dot{\Phi} d x=\int_{D_{T}} \frac{m r P_{1}^{\prime}}{P_{1}} S^{m-1}\left(\frac{P_{1}}{r^{2}} \sum_{i=1}^{3} Z_{i}^{2} \dot{\Phi}\right) \cdot r^{\mu} a(r) \partial_{r} S^{m} \dot{\Phi} d x \\
= & \int_{D_{T}} \frac{m r^{\mu+1} a(r) P_{1}^{\prime}}{P_{1}}\left\{\left[S^{m-1}\left(\frac{P_{1}}{r^{2}} \sum_{i=1}^{3} Z_{i}^{2} \dot{\Phi}\right)\right]^{2}\right.
\end{aligned}
$$




$$
\begin{gathered}
\left.+\left(\sum_{0 \leq l \leq m-1} C_{l m} \partial_{r} S^{l} \dot{\Phi}+r S^{m-1}\left(\mathcal{L} \dot{\Phi}-\frac{P_{2}}{r} \partial_{r} \dot{\Phi}\right)\right) S^{m-1}\left(\frac{P_{1}}{r^{2}} \sum_{i=1}^{3} Z_{i}^{2} \dot{\Phi}\right)\right\} d x \\
\leq \int_{D_{T}} \frac{m r^{\mu+1} a(r) P_{1}^{\prime}}{P_{1}}\left(\sum_{0 \leq l \leq m-1} C_{l m} \partial_{r} S^{l} \dot{\Phi}+r S^{m-1}\left(\mathcal{L} \dot{\Phi}-\frac{P_{2}}{r} \partial_{r} \dot{\Phi}\right)\right) S^{m-1}\left(\frac{P_{1}}{r^{2}} \sum_{i=1}^{3} Z_{i}^{2} \dot{\Phi}\right) d x \\
\left(\text { By } \frac{P_{1}^{\prime}(r)}{P_{1}(r)}<0\right)
\end{gathered}
$$

Note that $\sum_{0 \leq l \leq m-1} C_{l m} \partial_{r} S^{l} \dot{\Phi}-r S^{m-1}\left(\frac{P_{2}}{r} \partial_{r} \dot{\Phi}\right)$ only contains at most $m$-order derivatives of $\dot{\Phi}$, then we have by (5.2) for $l \leq m-1$

$$
\int_{D_{T}} r^{\mu-1-\delta}\left(\sum_{0 \leq l \leq m-1} C_{l m} \partial_{r} S^{l} \dot{\Phi}-r S^{m-1}\left(\frac{P_{2}}{r} \partial_{r} \dot{\Phi}\right)\right)^{2} d x \leq C \varepsilon^{2} .
$$

On the other hand, we have

$$
\left|S^{m-1} \mathcal{L} \dot{\Phi}\right| \leq C \varepsilon\left\{\sum_{0 \leq l \leq m}\left(r^{-\gamma}\left|S^{l} \partial_{r} \dot{\Phi}\right|+r^{-\sigma-2}\left|S^{l} Z \dot{\Phi}\right|\right)+\sum_{0 \leq l \leq m-1} r^{-2 \gamma}\left|S^{l} Z^{2} \dot{\Phi}\right|\right\} .
$$

Therefore, inserting (5.36)-(5.37) into (5.35) yields

$$
\begin{aligned}
& \int_{D_{T}} B_{2 m} \dot{\Phi} \cdot \mathcal{M} S^{m} \dot{\Phi} d x \leq C \varepsilon^{2}+C \varepsilon \int_{D_{T}} \sum_{l=0}^{m}\left(r^{\mu+1-2 \gamma+2 l}\left(\nabla_{x}^{l}\left(\frac{1}{r} Z \dot{\Phi}\right)\right)^{2}+r^{\mu-1-\delta+2 l}\left(\nabla_{x}^{l} \partial_{r} \dot{\Phi}\right)^{2}\right) d x \\
& \quad+C \varepsilon\left(\int_{D_{T}} r^{\mu-1-2 \gamma}\left|S^{m-1} Z^{2} \dot{\Phi}\right|^{2} d x\right)^{\frac{1}{2}}
\end{aligned}
$$

Consequently, inserting (5.9)-(5.10), (5.15)-(5.17), (5.28), (5.32) and (5.38) into (5.6), we can complete the proof of (5.3).

For the case of $m=0,(5.4)$ comes directly from Theorem 4.1, (5.10) and (5.15)-(5.17).

Based on Lemma 5.2 and the ingredients in Lemma 5.2, we now derive a series of estimates on the higher order derivatives of $\dot{\Phi}$.

Lemma 5.3. (Second order angular derivative estimates) Under the assumptions of Theorem 5.1, then

$$
\begin{aligned}
& T^{\mu} \int_{S_{T}}\left(\partial_{r} Z \dot{\Phi}\right)^{2} d S+T^{\mu-2 \gamma} \int_{S_{T}}\left(Z^{2} \dot{\Phi}\right)^{2} d S+\int_{D_{T}}\left(r^{\mu-1-\delta}\left(\partial_{r} Z \dot{\Phi}\right)^{2}+r^{\mu-1-2 \gamma}\left(Z^{2} \dot{\Phi}\right)^{2}\right) d x \\
& \leq C \varepsilon^{2}+C \varepsilon\left(T^{\mu} \int_{S_{T}}\left(\partial_{r} Z \dot{\Phi}\right)^{2} d S+T^{\mu-2 \gamma} \int_{S_{T}}\left(Z^{2} \dot{\Phi}\right)^{2} d S\right) \\
& \quad+C \varepsilon\left(\sum_{l=0}^{1} \int_{D_{T}} r^{\mu+1-2 \gamma+2 l}\left(\nabla_{x}^{l}\left(\frac{1}{r} Z \dot{\Phi}\right)\right)^{2}+r^{\mu-1-\delta+2 l}\left(\nabla_{x}^{l} \partial_{r} \dot{\Phi}\right)^{2} d x\right)
\end{aligned}
$$


where $0<\delta \leq \gamma-1$.

Remark 5.3. Lemma 5.3, together with Lemma 5.2 for $m=1$, yields (5.2) in the case of $k=1$.

Proof. Noting $\mathcal{L} Z \dot{\Phi}=Z \mathcal{L} \dot{\Phi}=Z \dot{f}$, then it follows from Theorem 4.1 that

$$
\begin{aligned}
& T^{\mu} \int_{S_{T}}\left|\partial_{r} Z \dot{\Phi}\right|^{2} d S+T^{\mu-2 \gamma} \int_{S_{T}}\left|Z^{2} \dot{\Phi}\right|^{2} d S+\int_{D_{T}}\left(r^{\mu-1-\delta}\left|\partial_{r} Z \dot{\Phi}\right|^{2}+r^{\mu-1-2 \gamma}\left|Z^{2} \dot{\Phi}\right|^{2}\right) d x \\
& \leq C \int_{D_{T}} Z \dot{f} \cdot \mathcal{M} Z \dot{\Phi} d x+C \varepsilon^{2}
\end{aligned}
$$

In order to estimate the term $\int_{D_{T}} Z \dot{f} \cdot \mathcal{M} Z \dot{\Phi} d x$ in the right hand side of (5.40), we rewrite $Z \dot{f}=D_{1}+D_{2}$ with

$$
\begin{aligned}
D_{1}= & f_{00} \partial_{r}^{2} Z \dot{\Phi}+\frac{1}{r^{2}} \sum_{1 \leq i, j \leq 3} f_{i j} Z_{i} Z_{j} Z \dot{\Phi}+\frac{1}{r} \sum_{i=1}^{3} f_{0 i} \partial_{r} Z_{i}(Z \dot{\Phi}), \\
D_{2}= & \frac{1}{r^{2}} \sum_{1 \leq i, j \leq 3} f_{i j}\left[Z, Z_{i} Z_{j}\right] \dot{\Phi}+\frac{1}{r} \sum_{i=1}^{3} f_{0 i}\left[Z, \partial_{r} Z_{i}\right] \dot{\Phi}+Z f_{00} \partial_{r}^{2} \dot{\Phi} \\
& +Z\left(\frac{1}{r^{2}} \sum_{1 \leq i, j \leq 3} f_{i j}\right) Z_{i} Z_{j} \dot{\Phi}+Z\left(\frac{1}{r} \sum_{i=1}^{3} f_{0 i}\right) \partial_{r} Z_{i} \dot{\Phi}+Z f_{0},
\end{aligned}
$$

where $D_{1}$ contains the third order derivatives of $\dot{\Phi}$, and $D_{2}$ is composed by the lower order (up to second order) derivative terms of $\dot{\Phi}$.

In this case, a direct computation yields

$$
\begin{aligned}
D_{1} & \cdot \mathcal{M} Z \dot{\Phi} \\
= & \partial_{r}\left(\frac{1}{2} r^{\mu} a(r) f_{00}\left(\partial_{r} Z \dot{\Phi}\right)^{2}-r^{\mu-2} a(r) \sum_{1 \leq i<j \leq 3} f_{i j} Z_{i} Z \dot{\Phi} Z_{j} Z \dot{\Phi}-\frac{1}{2} r^{\mu-2} a(r) \sum_{i=1}^{3} f_{i i}\left(Z_{i} Z \dot{\Phi}\right)^{2}\right) \\
& +\sum_{i=1}^{3} Z_{i}\left(\frac{1}{2} r^{\mu-1} a(r) f_{0 i}\left(\partial_{r} Z \dot{\Phi}\right)^{2}+r^{\mu-2} a(r) \partial_{r} Z \dot{\Phi} \sum_{j=1}^{3} f_{i j} Z_{j} Z \dot{\Phi}\right) \\
& -\frac{1}{2} \partial_{r}\left(r^{\mu} a(r) f_{00}\right)\left(\partial_{r} Z \dot{\Phi}\right)^{2}-\frac{1}{2} r^{\mu-1} a(r)\left(\partial_{r} Z \dot{\Phi}\right)^{2} \sum_{i=1}^{3} Z_{i} f_{0 i}+\sum_{i=1}^{3} \partial_{r}\left(\frac{1}{2} r^{\mu-2} a(r) f_{i i}\right)\left(Z_{i} Z \dot{\Phi}\right)^{2} \\
& +\sum_{1 \leq i<j \leq 3} \partial_{r}\left(r^{\mu-2} a(r) f_{i j}\right) Z_{i} Z \dot{\Phi} Z_{j} Z \dot{\Phi} .
\end{aligned}
$$

On the other hand, as in (5.14), it follows from the expressions of $f_{i j}, f_{0 i}$ and the boundary condition (3.4) that on $\Sigma$

$$
x_{2}\left(\frac{1}{2} r^{\mu-1} a(r) f_{02}\left(\partial_{r} Z \dot{\Phi}\right)^{2}+r^{\mu-2} a(r) \partial_{r} Z \dot{\Phi} \sum_{j=1}^{3} f_{2 j} Z_{j} Z \dot{\Phi}\right)
$$




$$
-x_{1}\left(\frac{1}{2} r^{\mu-1} a(r) f_{03}\left(\partial_{r} Z \dot{\Phi}\right)^{2}+r^{\mu-2} a(r) \partial_{r} Z \dot{\Phi} \sum_{j=1}^{3} f_{3 j} Z_{j} Z \dot{\Phi}\right)=0 .
$$

Thus, by integration by parts together with the expressions of $f_{i}$ and (5.1), we have

$$
\begin{aligned}
\left|\int_{D_{T}} D_{1} \cdot \mathcal{M} Z \dot{\Phi} d x\right| & \leq C \varepsilon^{2}+C \varepsilon\left(T^{\mu} \int_{S_{T}}\left(\partial_{r} Z \dot{\Phi}\right)^{2} d S+T^{\mu-2 \gamma} \int_{S_{T}}\left(Z^{2} \dot{\Phi}\right)^{2} d S\right) \\
& +C \varepsilon\left(\sum_{l=0}^{1} \int_{D_{T}} r^{\mu+1-2 \gamma+2 l}\left(\nabla_{x}^{l}\left(\frac{1}{r} Z \dot{\Phi}\right)\right)^{2}+r^{\mu-1-\delta+2 l}\left(\nabla_{x}^{l} \partial_{r} \dot{\Phi}\right)^{2} d x\right) .
\end{aligned}
$$

In addition, a direct computation yields

$$
\left|D_{2}\right| \leq C \varepsilon\left(r^{-2(\gamma-1)}\left|\partial_{r}^{2} \dot{\Phi}\right|+r^{-2(\gamma-1)}\left|\partial_{r} Z \dot{\Phi}\right|+r^{-2-\sigma}\left|Z^{2} \dot{\Phi}\right|\right),
$$

which implies

$$
\int_{D_{T}}\left|D_{2} \cdot \mathcal{M} Z \dot{\Phi}\right| d x \leq C \varepsilon \int_{D_{T}}\left(r^{\mu+1-\delta}\left(\partial_{r}^{2} \dot{\Phi}\right)^{2}+r^{\mu-1-\delta}\left(\partial_{r} Z \dot{\Phi}\right)^{2}+r^{\mu-1-2 \gamma}\left(Z^{2} \dot{\Phi}\right)^{2}\right) d x
$$

Substituting (5.43) and (5.45) into (5.40) yields (5.39), we then complete the proof of Lemma 5.3.

Lemma 5.4. (Third order angular derivative estimates) Under the assumptions of Theorem 5.1, then

$$
\begin{aligned}
& T^{\mu} \int_{S_{T}}\left(\partial_{r} Z^{2} \dot{\Phi}\right)^{2} d S+T^{\mu-2 \gamma} \int_{S_{T}}\left(Z^{3} \dot{\Phi}\right)^{2} d S+\int_{D_{T}}\left(r^{\mu-\gamma}\left(\partial_{r} Z^{2} \dot{\Phi}\right)^{2}+r^{\mu-1-2 \gamma}\left(Z^{3} \dot{\Phi}\right)^{2}\right) d x \\
& \leq C \varepsilon^{2}+C \varepsilon \int_{D_{T}} \sum_{l=0}^{2}\left(r^{\mu+1-2 \gamma+2 l}\left(\nabla_{x}^{l}\left(\frac{1}{r} Z \dot{\Phi}\right)\right)^{2}+r^{\mu-1-\delta+2 l}\left(\nabla_{x}^{l} \partial_{r} \dot{\Phi}\right)^{2}\right) d x \\
& \quad+C \varepsilon \sum_{l=0}^{2}\left(T^{\mu+2 l} \int_{S_{T}}\left(\nabla_{x}^{l} \partial_{r} \dot{\Phi}\right)^{2} d S+T^{\mu-2 \gamma+2 l} \int_{S_{T}}\left(\nabla_{x}^{l}\left(\frac{1}{r} Z \dot{\Phi}\right)\right)^{2} d S\right)
\end{aligned}
$$

where $0<\delta \leq \min \{\gamma-1,2 \sigma-2(\gamma-1)\}$ with $\sigma=\min \{1,2(\gamma-1)\}$.

Remark 5.4. Under the conditions of Lemma 5.4, as in the proof of Lemma 5.3, we have

$$
\begin{aligned}
& T^{\mu} \int_{S_{T}}\left(\partial_{r} S Z \dot{\Phi}\right)^{2} d S+T^{\mu-2 \gamma} \int_{S_{T}}(Z S Z \dot{\Phi})^{2} d S \\
& \quad+\int_{D_{T}}\left(r^{\mu-1-\delta}\left(\partial_{r} S Z \dot{\Phi}\right)^{2}+r^{\mu-1-2 \gamma}(Z S Z \dot{\Phi})^{2}\right) d x \\
& \leq C \varepsilon^{2}+C \varepsilon \int_{D_{T}} \sum_{l=0}^{2}\left(r^{\mu+1-2 \gamma+2 l}\left(\nabla_{x}^{l}\left(\frac{1}{r} Z \dot{\Phi}\right)\right)^{2}+r^{\mu-1-\delta+2 l}\left(\nabla_{x}^{l} \partial_{r} \dot{\Phi}\right)^{2}\right) d x
\end{aligned}
$$




$$
\begin{aligned}
& +C \varepsilon \sum_{l=0}^{2}\left(T^{\mu+2 l} \int_{S_{T}}\left(\nabla_{x}^{l} \partial_{r} \dot{\Phi}\right)^{2} d S+T^{\mu-2 \gamma+2 l} \int_{S_{T}}\left(\nabla_{x}^{l}\left(\frac{1}{r} Z \dot{\Phi}\right)\right)^{2} d S\right) \\
& +C \varepsilon\left(\int_{D_{T}}\left(r^{\mu-1-\delta}\left(\partial_{r} S Z \dot{\Phi}\right)^{2}+r^{\mu-1-2 \gamma}\left(Z^{3} \dot{\Phi}\right)^{2}\right) d x\right)^{\frac{1}{2}} .
\end{aligned}
$$

This, together with (5.46) and (5.3) in the case of $m=2$ and Remark 2.3, yields (5.2) for $k=2$ under the assumption that (5.2) holds for $k \leq 1$.

Proof. At first, we establish an analogous inequality to (5.46) for $\left(Z_{2}^{2}+Z_{3}^{2}\right) \dot{\Phi}$. Based on this together with the domain decomposition technique, we can complete the proof of (5.46).

It follows from (1.9) that on $\Sigma$

$$
\partial_{r}^{m} \partial_{\varphi} \Phi=0
$$

Differentiating (1.6) with respect to $\varphi$ and applying (5.47) yield on $\Sigma$

$$
\partial_{\varphi}\left(\sin \varphi \partial_{\varphi}^{2} \Phi\right)=0
$$

On the other hand, by a direct computation we have

$$
\partial_{\varphi}\left(Z_{2}^{2}+Z_{3}^{2}\right) \Phi=\left(\partial_{\varphi}^{3}+\cot \partial_{\varphi}^{2}-\csc ^{2} \varphi \partial_{\varphi}\right) \Phi .
$$

This, together with (5.47)-(5.48) and the definition of $\dot{\Phi}$, yields

$$
Z\left(Z_{2}^{2}+Z_{3}^{2}\right) \dot{\Phi}=0 \quad \text { on } \Sigma .
$$

Applying Theorem 4.1 to $\left(Z_{2}^{2}+Z_{3}^{2}\right) \dot{\Phi}$, one has for $\delta \leq \gamma-1$

$$
\begin{aligned}
& T^{\mu} \int_{S_{T}}\left(\partial_{r}\left(Z_{2}^{2}+Z_{3}^{2}\right) \dot{\Phi}\right)^{2} d S+T^{\mu-2 \gamma} \int_{S_{T}}\left(Z\left(Z_{2}^{2}+Z_{3}^{2}\right) \dot{\Phi}\right)^{2} d S \\
& \quad+\int_{D_{T}}\left(r^{\mu-\gamma}\left(\partial_{r}\left(Z_{2}^{2}+Z_{3}^{2}\right) \dot{\Phi}\right)^{2}+r^{\mu-1-2 \gamma}\left(Z\left(Z_{2}^{2}+Z_{3}^{2}\right) \dot{\Phi}\right)^{2}\right) d x \\
& \leq \int_{D_{T}} \mathcal{L}\left(Z_{2}^{2}+Z_{3}^{2}\right) \dot{\Phi} \cdot \mathcal{M}\left(Z_{2}^{2}+Z_{3}^{2}\right) \dot{\Phi} d x+C \varepsilon^{2} .
\end{aligned}
$$

By $\mathcal{L}\left(Z_{2}^{2}+Z_{3}^{2}\right) \dot{\Phi}=\left(Z_{2}^{2}+Z_{3}^{2}\right) \mathcal{L} \dot{\Phi}=\left(Z_{2}^{2}+Z_{3}^{2}\right) \dot{f}$ and the expression of $\dot{f}$, we have

$$
\mathcal{L}\left(Z_{2}^{2}+Z_{3}^{2}\right) \dot{\Phi}=K_{1}+K_{2}+K_{3}+K_{4}
$$

with

$$
\begin{aligned}
& K_{1}=f_{00} \partial_{r}^{2}\left(Z_{2}^{2}+Z_{3}^{2}\right) \dot{\Phi}+\frac{1}{r^{2}} \sum_{1 \leq i, j \leq 3} f_{i j} Z_{i} Z_{j}\left(Z_{2}^{2}+Z_{3}^{2}\right) \dot{\Phi}+\frac{1}{r} \sum_{i=1}^{3} f_{0 i} \partial_{r} Z_{i}\left(\left(Z_{2}^{2}+Z_{3}^{2}\right) \dot{\Phi}\right), \\
& K_{2}=\frac{1}{r^{2}} \sum_{1 \leq i, j \leq 3} f_{i j}\left[\left(Z_{2}^{2}+Z_{3}^{2}\right), Z_{i} Z_{j}\right] \dot{\Phi}+\frac{1}{r} \sum_{i=1}^{3} f_{0 i}\left[\left(Z_{2}^{2}+Z_{3}^{2}\right), \partial_{r} Z_{i}\right] \dot{\Phi}
\end{aligned}
$$




$$
\begin{aligned}
K_{3}= & \sum_{k=2}^{3} \sum_{l=1}^{2} C_{l}\left(Z_{k}^{l} f_{00} Z_{k}^{2-l} \partial_{r}^{2} \dot{\Phi}+Z_{k}^{l}\left(\frac{1}{r^{2}} \sum_{1 \leq i, j \leq 3} f_{i j}\right) Z_{k}^{2-l} Z_{i} Z_{j} \dot{\Phi}+Z_{k}^{l}\left(\frac{1}{r} \sum_{i=1}^{3} f_{0 i}\right) Z_{k}^{2-l} \partial_{r} Z_{i} \dot{\Phi}\right) \\
& +\left(Z_{2}^{2}+Z_{3}^{2}\right) f_{0}^{1}, \\
K_{4}= & \left(Z_{2}^{2}+Z_{3}^{2}\right) f_{0}^{2} .
\end{aligned}
$$

Next, we start to deal with each term $\int_{D_{T}} K_{i} \cdot \mathcal{M}\left(Z_{2}^{2}+Z_{3}^{2}\right) \dot{\Phi} d x(1 \leq i \leq 4)$.

(A) The estimate on $\int_{D_{T}} K_{1} \cdot \mathcal{M}\left(Z_{2}^{2}+Z_{3}^{2}\right) \dot{\Phi} d x$

Analogous to the treatment on $\int_{D_{T}} D_{1} \cdot \mathcal{M}\left(Z_{2}^{2}+Z_{3}^{2}\right) \dot{\Phi} d x$ in (5.41) and (5.43), we can obtain

$$
\begin{aligned}
& \left|\int_{D_{T}} K_{1} \cdot \mathcal{M}\left(Z_{2}^{2}+Z_{3}^{2}\right) \dot{\Phi} d x\right| \\
& \leq C \varepsilon^{2}+C \varepsilon\left(T^{\mu} \int_{S_{T}}\left(\partial_{r}\left(Z_{2}^{2}+Z_{3}^{2}\right) \dot{\Phi}\right)^{2} d S+T^{\mu-2 \gamma} \int_{S_{T}}\left(Z\left(Z_{2}^{2}+Z_{3}^{2}\right) \dot{\Phi}\right)^{2} d S\right. \\
& \left.\quad+\int_{D_{T}}\left(r^{\mu-1-\delta}\left(\partial_{r}\left(Z_{2}^{2}+Z_{3}^{2}\right) \dot{\Phi}\right)^{2}+r^{\mu-1-2 \gamma}\left(Z\left(Z_{2}^{2}+Z_{3}^{2}\right) \dot{\Phi}\right)^{2}\right) d x\right) .
\end{aligned}
$$

(B) The estimate on $\int_{D_{T}} K_{2} \cdot \mathcal{M}\left(Z_{2}^{2}+Z_{3}^{2}\right) \dot{\Phi} d x$

By the expressions of $f_{i j}$ and the assumption (5.1), it follows from a direct computation that

$$
\left|K_{2}\right| \leq C \varepsilon\left(r^{-2 \gamma}\left|Z^{3} \dot{\Phi}\right|+r^{-1-\sigma}\left|\partial_{r} Z^{2} \dot{\Phi}\right|\right)
$$

and

$\int_{D_{T}}\left|K_{2} \cdot \mathcal{M}\left(Z_{2}^{2}+Z_{3}^{2}\right) \dot{\Phi}\right| d x \leq C \varepsilon \int_{D_{T}} \sum_{l=0}^{2}\left(r^{\mu+1-2 \gamma+2 l}\left(\nabla_{x}^{l}\left(\frac{1}{r} Z \dot{\Phi}\right)\right)^{2}+r^{\mu-1-\delta+2 l}\left(\nabla_{x}^{l} \partial_{r} \dot{\Phi}\right)^{2}\right) d x$.

(C) The estimate on $\int_{D_{T}} K_{3} \cdot \mathcal{M}\left(Z_{2}^{2}+Z_{3}^{2}\right) \dot{\Phi} d x$

By the expressions of $f_{i j}$ and $f_{0}^{1}$, we know that $K_{3}$ only contains such terms: $\partial_{r}^{2} Z^{l} \dot{\Phi}(l=$ $0,1), \partial_{r} Z^{l} \dot{\Phi}(0 \leq l \leq 2)$ and $Z^{l} \dot{\Phi}(1 \leq l \leq 3)$ with suitably decayed coefficients. More concretely, by the assumption (5.1), we have

$$
\left|K_{3}\right| \leq C \varepsilon\left(r^{-2(\gamma-1)} \sum_{0 \leq l \leq 1}\left|\partial_{r}^{2} Z^{l} \dot{\Phi}\right|+r^{-\gamma} \sum_{0 \leq l \leq 2}\left|\partial_{r} Z^{l} \dot{\Phi}\right|+r^{-2 \gamma} \sum_{1 \leq l \leq 3}\left|Z^{l} \dot{\Phi}\right|\right),
$$

which implies

$$
\int_{D_{T}}\left|K_{3} \cdot \mathcal{M}\left(Z_{2}^{2}+Z_{3}^{2}\right) \dot{\Phi}\right| d x \leq C \varepsilon \int_{D_{T}} \sum_{l=0}^{2}\left(r^{\mu+1-2 \gamma+2 l}\left(\nabla_{x}^{l}\left(\frac{1}{r} Z \dot{\Phi}\right)\right)^{2}+r^{\mu-1-\delta+2 l}\left(\nabla_{x}^{l} \partial_{r} \dot{\Phi}\right)^{2}\right) d x .
$$


(D) The estimate on $\int_{D_{T}} K_{4} \cdot \mathcal{M}\left(Z_{2}^{2}+Z_{3}^{2}\right) \dot{\Phi} d x$

By the expression of $f_{0}^{2}$, we have

$$
K_{4}=K_{4}^{1}+K_{4}^{2}
$$

with

$$
\begin{aligned}
& K_{4}^{1}=\frac{\hat{U}}{r^{3}\left(\hat{U}^{2}-c^{2}(\hat{\rho})\right)} \sum_{k=2}^{3} \sum_{i, j=1}^{3}\left(C_{i j}\left(Z_{i} \dot{\Phi} Z_{k}^{2} Z_{j} \dot{\Phi}+Z_{k}^{2} Z_{i} \dot{\Phi} Z_{j} \dot{\Phi}\right)+2 Z_{k} C_{i j}\left(Z_{i} \dot{\Phi} Z_{k} Z_{j} \dot{\Phi}+Z_{k} Z_{i} \dot{\Phi} Z_{j} \dot{\Phi}\right)\right. \\
& \left.\quad+Z_{k}^{2} C_{i j} Z_{i} \dot{\Phi} Z_{j} \dot{\Phi}\right)+\frac{(\gamma-1) \hat{U}}{r^{3}\left(\hat{U}^{2}-c^{2}(\hat{\rho})\right)} \sum_{k=2}^{3} \sum_{i=1}^{3} Z_{i} \dot{\Phi} Z_{k}^{2} Z_{i} \dot{\Phi}, \\
& K_{4}^{2}=-\sum_{k=2}^{3}\left(\sum_{i, j=1}^{3} \frac{2 \hat{U}}{r^{3}\left(\hat{U}^{2}-c^{2}(\hat{\rho})\right)} C_{i j} Z_{k} Z_{i} \dot{\Phi} Z_{k} Z_{j} \dot{\Phi}+\sum_{i=1}^{3} \frac{2(\gamma-1) \hat{U}}{r^{3}\left(\hat{U}^{2}-c^{2}(\hat{\rho})\right)}\left(Z_{k} Z_{i} \dot{\Phi}\right)^{2}\right),
\end{aligned}
$$

where $K_{4}^{1}=O\left(\frac{Z \dot{\Phi} Z^{3} \dot{\Phi}}{r^{3}}\right)+O\left(\frac{Z \dot{\Phi} Z^{2} \dot{\Phi}}{r^{3}}\right)$, and $K_{4}^{2}=O\left(\frac{Z_{i} Z_{j} \dot{\Phi} Z_{k} Z_{l} \dot{\Phi}}{r^{3}}\right)$. Here we point out that $K_{4}^{1}$ can be easily estimated since $\frac{Z \dot{\Phi}}{r}$ admits a good decay rate in assumption (5.1). In fact, we have

$$
\left|K_{4}^{1}\right| \leq C r^{-3}|Z \dot{\Phi}| \sum_{l=0}^{2}\left|Z^{l} Z \dot{\Phi}\right| \leq C r^{-2-\sigma} \sum_{l=0}^{2}\left|Z^{l} Z \dot{\Phi}\right|
$$

and

$$
\int_{D_{T}}\left|K_{4}^{1} \cdot \mathcal{M}\left(Z_{2}^{2}+Z_{3}^{2}\right) \dot{\Phi}\right| d x \leq C \varepsilon \int_{D_{T}} \sum_{l=0}^{2}\left(r^{\mu+1-2 \gamma+2 l}\left(\nabla_{x}^{l}\left(\frac{1}{r} Z \dot{\Phi}\right)\right)^{2}+r^{\mu-1-\delta+2 l}\left(\nabla_{x}^{l} \partial_{r} \dot{\Phi}\right)^{2}\right) d x .
$$

Next we deal with $\int_{D_{T}}\left|K_{4}^{2} \cdot \mathcal{M}\left(Z_{2}^{2}+Z_{3}^{2}\right) \dot{\Phi}\right| d x$.

By Hölder inequality and Lemma 2.6-Lemma 2.7, we have for $\delta<2 \sigma-2(\gamma-1)$

$$
\begin{aligned}
& \left|r^{\mu-3} Z_{k} Z_{i} \dot{\Phi} Z_{k} Z_{j} \dot{\Phi} \partial_{r}\left(Z_{2}^{2}+Z_{3}^{2}\right) \dot{\Phi}\right|_{L_{1}\left(D_{T}\right)} \\
= & \left|r^{\frac{\delta+2(\gamma-1)-2 \sigma}{2}} \cdot r^{\frac{2 \gamma+2 \sigma-9}{4}} Z_{k} Z_{i} \dot{\Phi} \cdot r^{\frac{2 \gamma+2 \sigma-9}{4}} Z_{k} Z_{j} \dot{\Phi} \cdot r^{\frac{\mu-1-\delta}{2}} \partial_{r}\left(Z_{2}^{2}+Z_{3}^{2}\right) \dot{\Phi}\right|_{L_{1}\left(D_{T}\right)} \\
\leq & C\left|r^{\frac{2 \gamma+2 \sigma-9}{4}} Z_{k} Z_{i} \dot{\Phi}\right|_{L^{4}\left(D_{T}\right)}\left|r^{\frac{2 \gamma+2 \sigma-9}{4}} Z_{k} Z_{j} \dot{\Phi}\right|_{L^{4}\left(D_{T}\right)}\left|r^{\frac{\mu-1-\delta}{2}} \partial_{r}\left(Z_{2}^{2}+Z_{3}^{2}\right) \dot{\Phi}\right|_{L^{2}\left(D_{T}\right)} \\
\leq & C \varepsilon \sum_{l=0}^{2}\left(\left|r^{\frac{\mu-1-\delta+2 l}{2}} \nabla_{x}^{l} \partial_{r} \dot{\Phi}\right|_{L^{2}\left(D_{T}\right)}+\left|r^{\frac{\mu+1-2 \gamma+2 l}{2}} \nabla_{x}^{l}\left(\frac{1}{r} Z \dot{\Phi}\right)\right|_{L^{2}\left(D_{T}\right)}\right)\left|r^{\frac{\mu-1-\delta}{2}} \partial_{r}\left(Z_{2}^{2}+Z_{3}^{2}\right) \dot{\Phi}\right|_{L^{2}\left(D_{T}\right)} \\
\leq & C \varepsilon \sum_{l=0}^{2}\left(\left|r^{\frac{\mu-1-\delta+2 l}{2}} \nabla_{x}^{l} \partial_{r} \dot{\Phi}\right|_{L^{2}\left(D_{T}\right)}^{2}+\left|r^{\frac{\mu+1-2 \gamma+2 l}{2}} \nabla_{x}^{l}\left(\frac{1}{r} Z \dot{\Phi}\right)\right|_{L^{2}\left(D_{T}\right)}^{2}\right)
\end{aligned}
$$


which derives

$$
\int_{D_{T}}\left|K_{4}^{2} \cdot \mathcal{M}\left(Z_{2}^{2}+Z_{3}^{2}\right) \dot{\Phi}\right| d x \leq C \varepsilon \int_{D_{T}} \sum_{l=0}^{2}\left(r^{\mu+1-2 \gamma+2 l} \nabla_{x}^{l}\left(\frac{1}{r} Z \dot{\Phi}\right)^{2}+r^{\mu-1-\delta+2 l}\left(\nabla_{x}^{l} \partial_{r} \dot{\Phi}\right)^{2}\right) d x .
$$

Substituting (5.51), (5.52), (5.54)-(5.58) into (5.50) yields

$$
\begin{aligned}
& T^{\mu} \int_{S_{T}}\left(\partial_{r}\left(Z_{2}^{2}+Z_{3}^{2}\right) \dot{\Phi}\right)^{2} d S+T^{\mu-2 \gamma} \int_{S_{T}}\left(Z\left(Z_{2}^{2}+Z_{3}^{2}\right) \dot{\Phi}\right)^{2} d S \\
& \quad+\int_{D_{T}}\left(r^{\mu-\gamma}\left(\partial_{r}\left(Z_{2}^{2}+Z_{3}^{2}\right) \dot{\Phi}\right)^{2}+r^{\mu-1-2 \gamma}\left(Z\left(Z_{2}^{2}+Z_{3}^{2}\right) \dot{\Phi}\right)^{2}\right) d x \\
& \leq C \varepsilon^{2}+C \varepsilon \int_{D_{T}} \sum_{l=0}^{2}\left(r^{\mu+1-2 \gamma+2 l}\left(\nabla_{x}^{l}\left(\frac{1}{r} Z \dot{\Phi}\right)\right)^{2}+r^{\mu-1-\delta+2 l}\left(\nabla_{x}^{l} \partial_{r} \dot{\Phi}\right)^{2}\right) d x \\
& \quad+C \varepsilon\left(T^{\mu} \int_{S_{T}}\left(\partial_{r}\left(Z_{2}^{2}+Z_{3}^{2}\right) \dot{\Phi}\right)^{2} d S+T^{\mu-2 \gamma} \int_{S_{T}}\left(Z\left(Z_{2}^{2}+Z_{3}^{2}\right) \dot{\Phi}\right)^{2} d S\right) .
\end{aligned}
$$

By (5.59), we know that the estimates of $Z\left(Z_{2}^{2}+Z_{3}^{2}\right) \dot{\Phi}$ and $\partial_{r}\left(Z_{2}^{2}+Z_{3}^{2}\right) \dot{\Phi}$ on $S_{T}$ or $D_{T}$ have been established but such kinds of third order angular derivatives $Z^{3} \dot{\Phi}$ and $\partial_{r} Z^{2} \dot{\Phi}$ are not estimated. However, away from $\varphi=0$ in $\Omega$, we can get the estimates on the third order derivatives $Z^{3} \dot{\Phi}$ and $\partial_{r} Z^{2} \dot{\Phi}$. Indeed, for $\varphi>\frac{\varphi_{0}}{3}$, by Lemma 2.5 a direct computation yields

$$
\left\{\begin{array}{l}
\sum_{i, j, k=1}^{3}\left|Z_{i} Z_{j} Z_{k} \dot{\Phi}\right| \leq C\left(\sum_{i=1}^{3}\left|Z_{i} \sum_{j=1}^{3} Z_{j}^{2} \dot{\Phi}\right|+\sum_{i, j=1}^{3}\left|Z_{i} Z_{j} \dot{\Phi}\right|+\sum_{i=1}^{3}\left|Z_{i} \dot{\Phi}\right|\right) \\
\sum_{i, j=1}^{3}\left|\partial_{r} Z_{i} Z_{j} \dot{\Phi}\right| \leq C\left(\left|\partial_{r} \sum_{j=1}^{3} Z_{j}^{2} \dot{\Phi}\right|+\sum_{i=1}^{3}\left|\partial_{r} Z_{i} \dot{\Phi}\right|+\left|\partial_{r} \dot{\Phi}\right|\right) .
\end{array}\right.
$$

This, together with $Z_{1}^{k} \dot{\Phi} \equiv 0(k \in \mathbb{N}),(5.59)$ and Lemma 5.3, yields

$$
\begin{aligned}
& T^{\mu} \int_{S_{T} \cap\left\{\varphi \geq \frac{\varphi_{0}}{3}\right\}}\left(\partial_{r} Z^{2} \dot{\Phi}\right)^{2} d S+T^{\mu-2 \gamma} \int_{S_{T} \cap\left\{\varphi \geq \frac{\varphi_{0}}{3}\right\}}\left(Z^{3} \dot{\Phi}\right)^{2} d S \\
& \quad+\int_{D_{T} \cap\left\{\varphi \geq \frac{\varphi_{0}}{3}\right\}}\left(r^{\mu-\gamma}\left(\partial_{r} Z^{2} \dot{\Phi}\right)^{2}+r^{\mu-1-2 \gamma}\left(Z^{3} \dot{\Phi}\right)^{2}\right) d x \\
& \leq C \varepsilon^{2}+C \varepsilon \int_{D_{T}} \sum_{l=0}^{2}\left(r^{\mu+1-2 \gamma+2 l}\left(\nabla_{x}^{l}\left(\frac{1}{r} Z \dot{\Phi}\right)\right)^{2}+r^{\mu-1-\delta+2 l}\left(\nabla_{x}^{l} \partial_{r} \dot{\Phi}\right)^{2}\right) d x \\
& \quad+C \varepsilon\left(T^{\mu} \int_{S_{T}}\left(\partial_{r}\left(Z_{2}^{2}+Z_{3}^{2}\right) \dot{\Phi}\right)^{2} d S+T^{\mu-2 \gamma} \int_{S_{T}}\left(Z\left(Z_{2}^{2}+Z_{3}^{2}\right) \dot{\Phi}\right)^{2} d S\right) .
\end{aligned}
$$

To obtain the estimates of $Z^{3} \dot{\Phi}$ and $\partial_{r} Z^{2} \dot{\Phi}$ on the domain $\left\{\varphi \leq \frac{\varphi_{0}}{3}\right\}$, we will take a domain 
decomposition technique. Namely, we choose a smooth cut-off function $\chi(\varphi)$ as follows

$$
\chi(\varphi)=\left\{\begin{array}{l}
1, \quad \text { for } 0 \leq \varphi \leq \frac{\varphi_{0}}{3}, \\
0, \quad \text { for } \frac{2 \varphi_{0}}{3} \leq \varphi \leq \varphi_{0}, \\
\text { smooth connection, } \text { for } \frac{\varphi_{0}}{3} \leq \varphi \leq \frac{2 \varphi_{0}}{3}
\end{array}\right.
$$

such that $\chi(\varphi) \dot{\Phi}$ is studied.

Indeed, by Theorem 4.1 we have

$$
\begin{aligned}
& T^{\mu} \int_{S_{T}} \chi(\varphi)\left(\partial_{r} Z^{2} \dot{\Phi}\right)^{2} d S+T^{\mu-2 \gamma} \int_{S_{T}} \chi(\varphi)\left(Z^{3} \dot{\Phi}\right)^{2} d S \\
& \quad+\int_{D_{T}} \chi(\varphi)\left(r^{\mu-\gamma}\left(\partial_{r} Z^{2} \dot{\Phi}\right)^{2}+r^{\mu-1-2 \gamma}\left(Z^{3} \dot{\Phi}\right)^{2}\right) d x \\
& \leq C \\
& \quad\left(\int_{D_{T}} r^{\mu-1-2 \gamma}\left|\chi^{\prime}(\varphi)\right|\left(Z^{3} \dot{\Phi}\right)^{2} d x\right)^{\frac{1}{2}}\left(\int_{D_{T}} r^{\mu-\gamma}\left|\chi^{\prime}(\varphi)\right|\left(\partial_{r} Z^{2} \dot{\Phi}\right)^{2} d x\right)^{\frac{1}{2}} \\
& \quad+\int_{D_{T}} \mathcal{L} Z^{2} \dot{\Phi} \cdot \chi(\varphi) \mathcal{M} Z^{2} \dot{\Phi} d x+C \varepsilon^{2},
\end{aligned}
$$

here we point out that $\chi^{\prime}(\varphi)$ has a compact support away from $\varphi=0$, which implies that the first term in the right hand side of (5.62) can be estimated as in (5.61).

On the other hand, by the compact support property of $\chi(\varphi)$ away from $\varphi=0$, then completely similar to the treatment on $\int_{D_{T}} \mathcal{L}\left(Z_{2}^{2}+Z_{3}^{2}\right) \dot{\Phi} \cdot \mathcal{M}\left(Z_{2}^{2}+Z_{3}^{2}\right) \dot{\Phi} d x$ in (5.50), we can arrive at

$$
\begin{aligned}
& \int_{D_{T}} \mathcal{L} Z^{2} \dot{\Phi} \cdot \chi(\varphi) \mathcal{M} Z^{2} \dot{\Phi} d x \\
\leq & C \varepsilon^{2}+C \varepsilon \int_{D_{T}} \sum_{l=0}^{2}\left(r^{\mu+1-2 \gamma+2 l}\left(\nabla_{x}^{l}\left(\frac{1}{r} Z \dot{\Phi}\right)\right)^{2}+r^{\mu-1-\delta+2 l}\left(\nabla_{x}^{l} \partial_{r} \dot{\Phi}\right)^{2}\right) d x \\
& +C \varepsilon \sum_{l=0}^{2}\left(T^{\mu+2 l} \int_{S_{T}}\left(\nabla_{x}^{l} \partial_{r} \dot{\Phi}\right)^{2} d S+T^{\mu-2 \gamma+2 l} \int_{S_{T}}\left(\nabla_{x}^{l}\left(\frac{1}{r} Z \dot{\Phi}\right)\right)^{2} d S\right) .
\end{aligned}
$$

Finally, combining (5.61) and (5.62)-(5.63) yields the proof of (5.46).

Finally, we deal with the estimates of $\nabla_{x}^{4} \dot{\Phi}$.

Lemma 5.5. (Fourth order angular derivative estimates) Under the assumptions of Theorem 5.1, then

$$
\begin{aligned}
& T^{\mu} \int_{S_{T}}\left(\partial_{r} Z^{3} \dot{\Phi}\right)^{2} d S+T^{\mu-2 \gamma} \int_{S_{T}}\left(Z^{4} \dot{\Phi}\right)^{2} d S+\int_{D_{T}}\left(r^{\mu-\gamma}\left(\partial_{r} Z^{3} \dot{\Phi}\right)^{2}+r^{\mu-1-2 \gamma}\left(Z^{4} \dot{\Phi}\right)^{2}\right) d x \\
\leq & C \varepsilon^{2}+C \varepsilon \int_{D_{T}} \sum_{l=0}^{3}\left(r^{\mu+1-2 \gamma+2 l}\left(\nabla_{x}^{l}\left(\frac{1}{r} Z \dot{\Phi}\right)\right)^{2}+r^{\mu-1-\delta+2 l}\left(\nabla_{x}^{l} \partial_{r} \dot{\Phi}\right)^{2}\right) d x
\end{aligned}
$$


36

$$
+C \varepsilon \sum_{l=0}^{3}\left(T^{\mu+2 l} \int_{S_{T}}\left(\nabla_{x}^{l} \partial_{r} \dot{\Phi}\right)^{2} d S+T^{\mu-2 \gamma+2 l} \int_{S_{T}}\left(\nabla_{x}^{l}\left(\frac{1}{r} Z \dot{\Phi}\right)\right)^{2} d S\right),
$$

where $0<\delta \leq \min \{\gamma-1, \sigma-(\gamma-1)\}$ with $\sigma=\min \{1,2(\gamma-1)\}$.

Remark 5.5. Under the conditions of Lemma 5.5, as in the proofs of Lemma 5.3 and Lemma 5.4 respectively, we have

$$
\begin{aligned}
& T^{\mu} \int_{S_{T}}\left(\partial_{r} S^{2} Z \dot{\Phi}\right)^{2} d S+T^{\mu-2 \gamma} \int_{S_{T}}\left(Z S^{2} Z \dot{\Phi}\right)^{2} d S \\
& \quad+\int_{D_{T}}\left(r^{\mu-1-\delta}\left(\partial_{r} S^{2} Z \dot{\Phi}\right)^{2}+r^{\mu-1-2 \gamma}\left(Z S^{2} Z \dot{\Phi}\right)^{2}\right) d x \\
& \leq C \varepsilon^{2}+C \varepsilon \int_{D_{T}} \sum_{l=0}^{3}\left(r^{\mu+1-2 \gamma+2 l}\left(\nabla_{x}^{l}\left(\frac{1}{r} Z \dot{\Phi}\right)\right)^{2}+r^{\mu-1-\delta+2 l}\left(\nabla_{x}^{l} \partial_{r} \dot{\Phi}\right)^{2}\right) d x \\
& \quad+C \varepsilon \sum_{l=0}^{3}\left(T^{\mu+2 l} \int_{S_{T}}\left(\nabla_{x}^{l} \partial_{r} \dot{\Phi}\right)^{2} d S+T^{\mu-2 \gamma+2 l} \int_{S_{T}}\left(\nabla_{x}^{l}\left(\frac{1}{r} Z \dot{\Phi}\right)\right)^{2} d S\right) \\
& \quad+C \varepsilon\left(\int_{D_{T}}\left(r^{\mu-1-\delta}\left(\partial_{r} S^{2} Z \dot{\Phi}\right)^{2}+r^{\mu-1-2 \gamma}\left(S Z^{3} \dot{\Phi}\right)^{2}\right) d x\right)^{\frac{1}{2}}
\end{aligned}
$$

and

$$
\begin{aligned}
& T^{\mu} \int_{S_{T}}\left(\partial_{r} S Z^{2} \dot{\Phi}\right)^{2} d S+T^{\mu-2 \gamma} \int_{S_{T}}\left(Z S Z^{2} \dot{\Phi}\right)^{2} d S \\
& \quad+\int_{D_{T}}\left(r^{\mu-1-\delta}\left(\partial_{r} S Z^{2} \dot{\Phi}\right)^{2}+r^{\mu-1-2 \gamma}\left(Z S Z^{2} \dot{\Phi}\right)^{2}\right) d x \\
& \leq C \varepsilon^{2}+C \varepsilon \int_{D_{T}} \sum_{l=0}^{3}\left(r^{\mu+1-2 \gamma+2 l}\left(\nabla_{x}^{l}\left(\frac{1}{r} Z \dot{\Phi}\right)\right)^{2}+r^{\mu-1-\delta+2 l}\left(\nabla_{x}^{l} \partial_{r} \dot{\Phi}\right)^{2}\right) d x \\
& \quad+C \varepsilon \sum_{l=0}^{3}\left(T^{\mu+2 l} \int_{S_{T}}\left(\nabla_{x}^{l} \partial_{r} \dot{\Phi}\right)^{2} d S+T^{\mu-2 \gamma+2 l} \int_{S_{T}}\left(\nabla_{x}^{l}\left(\frac{1}{r} Z \dot{\Phi}\right)\right)^{2} d S\right) \\
& \quad+C \varepsilon\left(\int_{D_{T}}\left(r^{\mu-1-\delta}\left(\partial_{r} S Z^{2} \dot{\Phi}\right)^{2}+r^{\mu-1-2 \gamma}\left(Z^{4} \dot{\Phi}\right)^{2}\right) d x\right)^{\frac{1}{2}}
\end{aligned}
$$

This, together with (5.64) and (5.3) in the case of $m=3$ and Remark 2.3, yields (5.2) for $k=3$.

Proof. As in Lemma 5.4, at first we establish an analogous inequality to (5.53) for $Z\left(Z_{2}^{2}+\right.$ $\left.Z_{3}^{2}\right) \dot{\Phi}$. Based on this together with the domain decomposition technique, we can complete the proof of (5.64).

Note that

$$
\partial_{r} Z\left(Z_{2}^{2}+Z_{3}^{2}\right) \dot{\Phi}=0 \quad \text { on } \varphi=\varphi_{0}
$$


Then applying Theorem 4.1 to $Z\left(Z_{2}^{2}+Z_{3}^{2}\right) \dot{\Phi}$ yields

$$
\begin{aligned}
& T^{\mu} \int_{S_{T}}\left(\partial_{r} Z\left(Z_{2}^{2}+Z_{3}^{2}\right) \dot{\Phi}\right)^{2} d S+T^{\mu-2 \gamma} \int_{S_{T}}\left(Z^{2}\left(Z_{2}^{2}+Z_{3}^{2}\right) \dot{\Phi}\right)^{2} d S \\
& \quad+\int_{D_{T}}\left(r^{\mu-\gamma}\left(\partial_{r} Z\left(Z_{2}^{2}+Z_{3}^{2}\right) \dot{\Phi}\right)^{2}+r^{\mu-1-2 \gamma}\left(Z^{2}\left(Z_{2}^{2}+Z_{3}^{2}\right) \dot{\Phi}\right)^{2}\right) d x \\
& \leq C \int_{D_{T}} \mathcal{L} Z\left(Z_{2}^{2}+Z_{3}^{2}\right) \dot{\Phi} \cdot \mathcal{M} Z\left(Z_{2}^{2}+Z_{3}^{2}\right) \dot{\Phi} d x+C \varepsilon^{2}
\end{aligned}
$$

By $\mathcal{L} Z\left(Z_{2}^{2}+Z_{3}^{2}\right) \dot{\Phi}=Z\left(Z_{2}^{2}+Z_{3}^{2}\right) \mathcal{L} \dot{\Phi}=Z\left(Z_{2}^{2}+Z_{3}^{2}\right) \dot{f}$ and the expression of $\dot{f}$, we have

$$
\mathcal{L} Z\left(Z_{2}^{2}+Z_{3}^{2}\right) \dot{\Phi}=M_{1}+M_{2}+M_{3}+M_{4}
$$

with

$$
\begin{aligned}
M_{1}= & f_{00} \partial_{r}^{2} Z\left(Z_{2}^{2}+Z_{3}^{2}\right) \dot{\Phi}+\frac{1}{r^{2}} \sum_{1 \leq i, j \leq 3} f_{i j} Z_{i} Z_{j} Z\left(Z_{2}^{2}+Z_{3}^{2}\right) \dot{\Phi}+\frac{1}{r} \sum_{i=1}^{3} f_{0 i} \partial_{r} Z_{i} Z\left(Z_{2}^{2}+Z_{3}^{2}\right) \dot{\Phi}, \\
M_{2}= & \frac{1}{r^{2}} \sum_{1 \leq i, j \leq 3} f_{i j}\left[Z\left(Z_{2}^{2}+Z_{3}^{2}\right), Z_{i} Z_{j}\right] \dot{\Phi}+\frac{1}{r} \sum_{i=1}^{3} f_{0 i}\left[Z\left(Z_{2}^{2}+Z_{3}^{2}\right), \partial_{r} Z_{i}\right] \dot{\Phi}, \\
M_{3}= & \sum_{k=2}^{3} \sum_{l=1}^{2} C_{l} Z\left(Z_{k}^{l} f_{00} Z_{k}^{2-l} \partial_{r}^{2} \dot{\Phi}+\sum_{1 \leq i, j \leq 3} Z_{k}^{l}\left(\frac{1}{r^{2}} f_{i j}\right) Z_{k}^{2-l} Z_{i} Z_{j} \dot{\Phi}+\sum_{i=1}^{3} Z_{k}^{l}\left(\frac{1}{r} f_{0 i}\right) Z_{k}^{2-l} \partial_{r} Z_{i} \dot{\Phi}\right) \\
& +\left(Z f_{00} Z_{k}^{2} \partial_{r}^{2} \dot{\Phi}+\sum_{k=2}^{3} \sum_{1 \leq i, j \leq 3} Z\left(\frac{1}{r^{2}} f_{i j}\right) Z_{k}^{2} Z_{i} Z_{j} \dot{\Phi}+\sum_{i=1}^{3} Z\left(\frac{1}{r} f_{0 i}\right) Z_{k}^{2} \partial_{r} Z_{i} \dot{\Phi}\right) \\
& \quad+Z\left(Z_{2}^{2}+Z_{3}^{2}\right) f_{0}^{1},
\end{aligned}
$$

For notational simplifications, we rewrite $M_{3}$ and $M_{4}$ as follows

$$
\begin{aligned}
M_{3}= & \sum{ }_{l_{1}+l_{2} \leq 3, l_{1} \geq 1} C_{l_{1} l_{2}}\left(Z^{l_{1}} f_{00} Z^{l_{2}} \partial_{r}^{2} \dot{\Phi}+\sum_{1 \leq i, j \leq 3} Z^{l_{1}}\left(\frac{1}{r^{2}} f_{i j}\right) Z^{l_{2}} Z_{i} Z_{j} \dot{\Phi}+\sum_{i=1}^{3} Z^{l_{1}}\left(\frac{1}{r} f_{0 i}\right) Z^{l_{2}} \partial_{r} Z_{i} \dot{\Phi}\right) \\
& +Z^{3} f_{0}^{1}, \\
M_{4}= & Z^{3} f_{0}^{2} .
\end{aligned}
$$

Next, we treat each term $\int_{D_{T}} M_{i} \cdot \mathcal{M} Z\left(Z_{2}^{2}+Z_{3}^{2}\right) \dot{\Phi} d x(1 \leq i \leq 4)$ respectively.

(A) The estimate on $\int_{D_{T}} M_{1} \cdot \mathcal{M} Z\left(Z_{2}^{2}+Z_{3}^{2}\right) \dot{\Phi} d x$

As in (5.52), we can obtain

$$
\left|\int_{D_{T}} M_{1} \cdot \mathcal{M} Z\left(Z_{2}^{2}+Z_{3}^{2}\right) \dot{\Phi} d x\right| \leq C \varepsilon^{2}+C \varepsilon\left(T^{\mu} \int_{S_{T}}\left(\partial_{r} Z\left(Z_{2}^{2}+Z_{3}^{2}\right) \dot{\Phi}\right)^{2} d S\right.
$$




$$
\begin{aligned}
& +T^{\mu-2 \gamma} \int_{S_{T}}\left(Z^{2}\left(Z_{2}^{2}+Z_{3}^{2}\right) \dot{\Phi}\right)^{2} d S+\int_{D_{T}}\left(r^{\mu-1-\delta}\left(\partial_{r} Z\left(Z_{2}^{2}+Z_{3}^{2}\right) \dot{\Phi}\right)^{2}\right. \\
& \left.\left.+r^{\mu-1-2 \gamma}\left(Z^{2}\left(Z_{2}^{2}+Z_{3}^{2}\right) \dot{\Phi}\right)^{2}\right) d x\right) .
\end{aligned}
$$

(B) The estimate on $\int_{D_{T}} M_{2} \cdot \mathcal{M} Z\left(Z_{2}^{2}+Z_{3}^{2}\right) \dot{\Phi} d x$

It follows from the expressions of $f_{i j},(5.1)$ and a direct computation that

$$
\left|M_{2}\right| \leq C \varepsilon\left(r^{-2 \gamma}\left|Z^{4} \dot{\Phi}\right|+r^{-\sigma-1}\left|\partial_{r} Z^{3} \dot{\Phi}\right|\right)
$$

and

$\int_{D_{T}}\left|M_{2} \cdot \mathcal{M} Z\left(Z_{2}^{2}+Z_{3}^{2}\right) \dot{\Phi}\right| d x \leq C \varepsilon \int_{D_{T}} \sum_{l=0}^{3}\left(r^{\mu+1-2 \gamma+2 l}\left(\nabla_{x}^{l}\left(\frac{1}{r} Z \dot{\Phi}\right)\right)^{2}+r^{\mu-1-\delta+2 l}\left(\nabla_{x}^{l} \partial_{r} \dot{\Phi}\right)^{2}\right) d x$.

(C) The estimate on $\int_{D_{T}} M_{3} \cdot \mathcal{M} Z\left(Z_{2}^{2}+Z_{3}^{2}\right) \dot{\Phi} d x$

Due to the lack of $L^{\infty}$ assumptions on the third order derivatives of $\dot{\Phi}$ in (5.1), we will decompose $M_{3}$ as follows

$$
M_{3}=M_{3}^{1}+M_{3}^{2},
$$

where $M_{3}^{1}$ is linear with respect to the third and fourth order derivatives of $\dot{\Phi}, M_{3}^{2}$ contains the products of two third order derivatives of $\dot{\Phi}$, which is required to be specially treated and admits the following concrete expression:

$$
\begin{aligned}
M_{3}^{2}= & -\frac{C_{12}}{\left(\hat{U}^{2}-c^{2}(\hat{\rho})\right)}\left\{\left((\gamma+1) \hat{U} \partial_{r} Z^{2} \dot{\Phi}+(\gamma+1) \partial_{r} \dot{\Phi} \partial_{r} Z^{2} \dot{\Phi}+\frac{\gamma-1}{r^{2}} \sum_{i=1}^{3} Z_{i} \dot{\Phi} Z^{2} Z_{i} \dot{\Phi}\right) \partial_{r}^{2} Z \dot{\Phi}\right. \\
& -\frac{\gamma-1}{r^{2}} \sum_{i=1}^{3}\left(\partial_{r} \dot{\Phi} \partial_{r} Z^{2} \dot{\Phi}-\hat{U} \partial_{r} Z^{2} \dot{\Phi}+\frac{1}{r^{2}} \sum_{k=1}^{3} Z_{k} \dot{\Phi} Z^{2} Z_{k} \dot{\Phi}-\frac{2}{r^{2}(\gamma-1)} Z_{i} \dot{\Phi} Z^{2} Z_{i} \dot{\Phi}\right) Z Z_{i}^{2} \dot{\Phi} \\
& \left.\left.+\frac{1}{r^{4}} \sum_{1 \leq i \neq j \leq 3} Z_{i} \dot{\Phi} Z^{2} Z_{j} \dot{\Phi} Z Z_{i} Z_{j} \dot{\Phi}+\frac{1}{r^{2}} \sum_{i=1}^{3}\left(\left(\hat{U}+\partial_{r} \dot{\Phi}\right) Z^{2} Z_{i} \dot{\Phi}+Z_{i} \dot{\Phi} \partial_{r} Z^{2} \dot{\Phi}\right) \partial_{r} Z Z_{i} \dot{\Phi}\right)\right\}
\end{aligned}
$$

By (5.1) and a direct but tedious computation, we can arrive at

$$
\left|M_{3}^{1}\right| \leq C \varepsilon\left(r^{-2(\gamma-1)} \sum_{0 \leq i \leq 2}\left|\partial_{r}^{2} Z^{i} \dot{\Phi}\right|+r^{-\delta-1} \sum_{0 \leq i \leq 3}\left|\partial_{r} Z^{i} \dot{\Phi}\right|+r^{-2-\sigma} \sum_{1 \leq i \leq 4}\left|Z^{i} \dot{\Phi}\right|\right),
$$

which yields

$$
\int_{D_{T}}\left|M_{3}^{1} \cdot \mathcal{M} Z\left(Z_{2}^{2}+Z_{3}^{2}\right) \dot{\Phi}\right| d x \leq C \varepsilon \int_{D_{T}} \sum_{l=0}^{3}\left(r^{\mu+1-2 \gamma+2 l}\left(\nabla_{x}^{l}\left(\frac{1}{r} Z \dot{\Phi}\right)\right)^{2}+r^{\mu-1-\delta+2 l}\left(\nabla_{x}^{l} \partial_{r} \dot{\Phi}\right)^{2}\right) d x
$$


Next, we focus on the treatment of $\int_{D_{T}}\left|M_{3}^{2} \cdot \mathcal{M} Z\left(Z_{2}^{2}+Z_{3}^{2}\right) \dot{\Phi}\right| d x$.

By the expression of $M_{3}^{2}$, one knows that the typical terms in $M_{3}^{2} \cdot \mathcal{M} Z\left(Z_{2}^{2}+Z_{3}^{2}\right) \dot{\Phi}$ are respectively:

$r^{\mu} \partial_{r} Z^{2} \dot{\Phi} \partial_{r}^{2} Z \dot{\Phi} \partial_{r} Z\left(Z_{2}^{2}+Z_{3}^{2}\right) \dot{\Phi}, r^{\mu-2} Z \dot{\Phi} Z^{3} \dot{\Phi} \partial_{r}^{2} Z \dot{\Phi} \partial_{r} Z\left(Z_{2}^{2}+Z_{3}^{2}\right) \dot{\Phi}, r^{\mu-2} \partial_{r} Z^{2} \dot{\Phi} Z^{3} \dot{\Phi} \partial_{r} Z\left(Z_{2}^{2}+\right.$ $\left.Z_{3}^{2}\right) \dot{\Phi}$

$r^{\mu-4} Z \dot{\Phi}\left(Z^{3} \dot{\Phi}\right)^{2} \partial_{r} Z\left(Z_{2}^{2}+Z_{3}^{2}\right) \dot{\Phi}, r^{\mu-2} Z^{3} \dot{\Phi} \partial_{r} Z^{2} \dot{\Phi} \partial_{r} Z\left(Z_{2}^{2}+Z_{3}^{2}\right) \dot{\Phi}$ and $r^{\mu-2} Z \dot{\Phi}\left(\partial_{r} Z^{2} \dot{\Phi}\right)^{2} \partial_{r} Z\left(Z_{2}^{2}+\right.$ $\left.Z_{3}^{2}\right) \dot{\Phi}$.

By the interpolation inequalities in Corollary 2.3 and Lemma 2.6, the terms mentioned above can be treated directly. Indeed, we have

(i)

$$
\begin{aligned}
& \left|r^{\mu} \partial_{r} Z^{2} \dot{\Phi} \partial_{r}^{2} Z \dot{\Phi} \partial_{r} Z\left(Z_{2}^{2}+Z_{3}^{2}\right) \dot{\Phi}\right|_{L^{1}\left(D_{T}\right)} \\
\leq & C\left|r^{\delta-2(\gamma-1)} \cdot r^{\frac{8 \gamma-11-\delta}{4}} \partial_{r} Z^{2} \dot{\Phi} \cdot r^{\frac{8-7-\delta}{4}} \partial_{r}^{2} Z \dot{\Phi} \cdot r^{\frac{\mu-1-\delta}{2}} \partial_{r} Z^{3} \dot{\Phi}\right|_{L_{1}\left(D_{T}\right)} \\
\leq & C\left|r^{\frac{8 \gamma-11-\delta}{4}} \partial_{r}^{2} Z \dot{\Phi}\right|_{L^{4}\left(D_{T}\right)}\left|r^{\frac{8 \gamma-7-\delta}{4}} \partial_{r}^{2} Z \dot{\Phi}\right|_{L^{4}\left(D_{T}\right)}\left|r^{\frac{\mu-1-\delta}{2}} \partial_{r} Z^{3} \dot{\Phi}\right|_{L^{2}\left(D_{T}\right)} . \quad(\text { By } \delta \leq \gamma-1)
\end{aligned}
$$

(ii)

$$
\begin{aligned}
& \left|r^{\mu-2} Z \dot{\Phi} Z^{3} \dot{\Phi} \partial_{r}^{2} Z \dot{\Phi} \partial_{r} Z\left(Z_{2}^{2}+Z_{3}^{2}\right) \dot{\Phi}\right|_{L^{1}\left(D_{T}\right)} \\
\leq & \left|r^{\mu-1-\sigma} Z^{3} \dot{\Phi} \partial_{r}^{2} Z \dot{\Phi} \partial_{r} Z\left(Z_{2}^{2}+Z_{3}^{2}\right) \dot{\Phi}\right|_{L_{1}\left(D_{T}\right)} \quad \text { (By assumption (5.1)) } \\
\leq & C\left|r^{\frac{3 \delta-(\gamma-1)-\sigma}{4}} \cdot r^{\frac{4 \gamma-11}{4}} Z^{3} \dot{\Phi} \cdot r^{\frac{8 \gamma-7-\delta}{4}} \partial_{r}^{2} Z \dot{\Phi} \cdot r^{\frac{\mu-1-\delta}{2}} \partial_{r} Z^{3} \dot{\Phi}\right|_{L_{1}\left(D_{T}\right)} \\
\leq & C\left|r^{\frac{4 \gamma-11}{4}} Z^{3} \dot{\Phi}\right|_{L^{4}\left(D_{T}\right)}\left|r^{\frac{8 \gamma-7-\delta}{4}} \partial_{r}^{2} Z \dot{\Phi}\right|_{L^{4}\left(D_{T}\right)}\left|r^{\frac{\mu-1-\delta}{2}} \partial_{r} Z^{3} \dot{\Phi}\right|_{L^{2}\left(D_{T}\right)},
\end{aligned}
$$

here we have used $0<\delta \leq \min (\gamma-1, \sigma-(\gamma-1))$ and $\sigma=\min (1,2(\gamma-1))$, which derives $3 \delta-(\gamma-1)-\sigma \leq 0$.

(iii)

$$
\begin{aligned}
& \left|r^{\mu-2} \partial_{r} Z^{2} \dot{\Phi} Z^{3} \dot{\Phi} \partial_{r} Z\left(Z_{2}^{2}+Z_{3}^{2}\right) \dot{\Phi}\right|_{L_{1}\left(D_{T}\right)} \\
= & \left|r^{\frac{3 \delta-4(\gamma-1)}{4}} \cdot r^{\frac{8 \gamma-11-\delta}{4}} \partial_{r} Z^{2} \dot{\Phi} \cdot r^{\frac{4 \gamma-11}{4}} Z^{3} \dot{\Phi} \cdot r^{\frac{\mu-1-\delta}{2}} \partial_{r} Z^{3} \dot{\Phi}\right|_{L^{1}\left(D_{T}\right)} \\
\leq & C\left|r^{\frac{8 \gamma-11-\delta}{4}} \partial_{r} Z^{2} \dot{\Phi}\right|_{L^{4}\left(D_{T}\right)}\left|r^{\frac{4 \gamma-11}{4}} Z^{3} \dot{\Phi}\right|_{L^{4}\left(D_{T}\right)}\left|r^{\frac{\mu-1-\delta}{2}} \partial_{r} Z^{3} \dot{\Phi}\right|_{L^{2}\left(D_{T}\right)} . \quad(\text { By } \delta \leq \gamma-1)
\end{aligned}
$$

(iv)

$$
\begin{aligned}
& \left|r^{\mu-4} Z \dot{\Phi}\left(Z^{3} \dot{\Phi}\right)^{2} \partial_{r} Z\left(Z_{2}^{2}+Z_{3}^{2}\right) \dot{\Phi}\right|_{L^{1}\left(D_{T}\right)} \\
\leq & \left|r^{\mu-3-\sigma}\left(Z^{3} \dot{\Phi}\right)^{2} \partial_{r} Z\left(Z_{2}^{2}+Z_{3}^{2}\right) \dot{\Phi}\right|_{L_{1}\left(D_{T}\right)} \quad(\text { By assumption }(5.1)) \\
= & \left|r^{\frac{\delta-2 \sigma}{2}} \cdot\left(r^{\frac{4 \gamma-11}{4}} Z^{3} \dot{\Phi}\right)^{2} \cdot r^{\frac{\mu-1-\delta}{2}} \partial_{r} Z^{3} \dot{\Phi}\right|_{L_{1}\left(D_{T}\right)} \\
\leq & C\left|r^{\frac{4 \gamma-11}{4}} Z^{3} \dot{\Phi}\right|_{L^{4}\left(D_{T}\right)}^{2}\left|r^{\frac{\mu-1-\delta}{2}} \partial_{r} Z^{3} \dot{\Phi}\right|_{L^{2}\left(D_{T}\right)} . \quad(\text { By } \delta \leq \gamma-1 \leq \sigma)
\end{aligned}
$$

(v)

$$
\left|r^{\mu-2} Z^{3} \dot{\Phi} \partial_{r} Z^{2} \dot{\Phi} \partial_{r} Z\left(Z_{2}^{2}+Z_{3}^{2}\right) \dot{\Phi}\right|_{L_{1}\left(D_{T}\right)}
$$


40

$$
\begin{aligned}
& =\left|r^{\frac{3 \delta-4(\gamma-1)}{4}} \cdot r^{\frac{4 \gamma-11}{4}} Z^{3} \dot{\Phi} \cdot r^{\frac{8 \gamma-11-\delta}{4}} \partial_{r} Z^{2} \dot{\Phi} \cdot r^{\frac{\mu-1-\delta}{2}} \partial_{r} Z^{3} \dot{\Phi}\right|_{L_{1}\left(D_{T}\right)} \\
& \leq C\left|r^{\frac{4 \gamma-11}{4}} Z^{3} \dot{\Phi}\right|_{L^{4}\left(D_{T}\right)}\left|r^{\frac{8 \gamma-11-\delta}{4}} \partial_{r} Z^{2} \dot{\Phi}\right|_{L^{4}\left(D_{T}\right)}\left|r^{\frac{\mu-1-\delta}{2}} \partial_{r} Z^{3} \dot{\Phi}\right|_{L^{2}\left(D_{T}\right)} . \quad(\text { By } \delta \leq \gamma-1)
\end{aligned}
$$

(vi)

$$
\begin{aligned}
& \left|r^{\mu-2} Z \dot{\Phi}\left(\partial_{r} Z^{2} \dot{\Phi}\right)^{2} \partial_{r} Z\left(Z_{2}^{2}+Z_{3}^{2}\right) \dot{\Phi}\right|_{L^{1}\left(D_{T}\right)} \\
\leq & \left|r^{\mu-1-\sigma}\left(\partial_{r} Z^{2} \dot{\Phi}\right)^{2} \partial_{r} Z\left(Z_{2}^{2}+Z_{3}^{2}\right) \dot{\Phi}\right|_{L_{1}\left(D_{T}\right)} \quad(\text { By assumption (5.1)) } \\
= & \left|r^{\delta-2(\gamma-1)-\sigma} \cdot\left(r^{\frac{8 \gamma-11-\delta}{4}} \partial_{r} Z^{2} \dot{\Phi}\right)^{2} \cdot r^{\frac{\mu-1-\delta}{2}} \partial_{r} Z^{3} \dot{\Phi}\right|_{L_{1}\left(D_{T}\right)} \\
\leq & C\left|r^{\frac{8 \gamma-11-\delta}{4}} \partial_{r} Z^{2} \dot{\Phi}\right|_{L^{4}\left(D_{T}\right)}^{2}\left|r^{\frac{\mu-1-\delta}{2}} \partial_{r} Z^{3} \dot{\Phi}\right|_{L^{2}\left(D_{T}\right)} . \quad(\text { By } \delta \leq \gamma-1)
\end{aligned}
$$

Substituting those estimates above into $\int_{D_{T}}\left|M_{3}^{2} \cdot \mathcal{M} Z\left(Z_{2}^{2}+Z_{3}^{2}\right) \dot{\Phi}\right| d x$ and applying Lemma 2.6-Lemma 2.7 yield

$$
\int_{D_{T}}\left|M_{3}^{2} \cdot \mathcal{M} Z\left(Z_{2}^{2}+Z_{3}^{2}\right) \dot{\Phi}\right| d x \leq C \varepsilon \int_{D_{T}} \sum_{l=0}^{3}\left(r^{\mu-1-\delta+2 l}\left(\nabla_{x}^{l} \partial_{r} \dot{\Phi}\right)^{2}+r^{\mu+1-2 \gamma+2 l}\left(\nabla_{x}^{l}\left(\frac{1}{r} Z \dot{\Phi}\right)\right)^{2}\right) d x
$$

By (5.70) and (5.71), we have

$$
\int_{D_{T}}\left|M_{3} \cdot \mathcal{M} Z\left(Z_{2}^{2}+Z_{3}^{2}\right) \dot{\Phi}\right| d x \leq C \varepsilon \int_{D_{T}} \sum_{l=0}^{3}\left(r^{\mu+1-2 \gamma+2 l}\left(\nabla_{x}^{l}\left(\frac{1}{r} Z \dot{\Phi}\right)\right)^{2}+r^{\mu-1-\delta+2 l}\left(\nabla_{x}^{l} \partial_{r} \dot{\Phi}\right)^{2}\right) d x .
$$

(D) The estimate on $\int_{D_{T}} M_{4} \cdot \mathcal{M} Z\left(Z_{2}^{2}+Z_{3}^{2}\right) \dot{\Phi} d x$

Note that

$$
\begin{aligned}
M_{4}= & -\frac{\hat{U}}{r^{3}} \sum_{i, j=1}^{3} C_{i j}^{\prime} Z Z_{i} \dot{\Phi} Z Z_{j} \dot{\Phi}-\frac{\hat{U}}{r^{3}} \sum_{i, j=1}^{3} \tilde{C}_{i j} Z Z_{i} \dot{\Phi} Z^{2} Z_{j} \dot{\Phi}+\frac{6(\gamma-1)}{r^{3}} \hat{U} \sum_{i=1}^{3} Z Z_{i} \dot{\Phi} Z Z_{i}^{2} \dot{\Phi} \\
& +\{\text { left terms }\} \\
\equiv & M_{4}^{1}+M_{4}^{2}
\end{aligned}
$$

and

$$
\left|M_{4}^{2}\right| \leq \frac{C}{r^{3}}|Z \dot{\Phi}| \sum_{i=1}^{4}\left|Z^{i} \dot{\Phi}\right|
$$

On the other hand, due to

$$
\begin{aligned}
& \left|r^{\mu-3}\left(Z^{2} \dot{\Phi}\right)^{2} \partial_{r} Z\left(Z_{2}^{2}+Z_{3}^{2}\right) \dot{\Phi}\right|_{L_{1}\left(D_{T}\right)} \\
\leq & \left|r^{\frac{\delta+2(\gamma-1)-2 \sigma}{2}} \cdot\left(r^{\frac{2 \gamma+2 \sigma-9}{4}} Z^{2} \dot{\Phi}\right)^{2} \cdot r^{\frac{\mu-1-\delta}{2}} \partial_{r} Z_{k}\left(Z_{2}^{2}+Z_{3}^{2}\right) \dot{\Phi}\right|_{L_{1}\left(D_{T}\right)}
\end{aligned}
$$




$$
\begin{aligned}
& \leq C\left|r^{\frac{2 \gamma+2 \sigma-9}{4}} Z^{2} \dot{\Phi}\right|_{L^{4}\left(D_{T}\right)}^{2}\left|r^{\frac{\mu-1-\delta}{2}} \partial_{r} Z_{k}\left(Z_{2}^{2}+Z_{3}^{2}\right) \dot{\Phi}\right|_{L^{2}\left(D_{T}\right)}, \quad(\text { By } \delta<2(\sigma-(\gamma-1))) \\
& \left|r^{\mu-3} Z^{2} \dot{\Phi} Z^{3} \dot{\Phi} \partial_{r} Z\left(Z_{2}^{2}+Z_{3}^{2}\right) \dot{\Phi}\right|_{L_{1}\left(D_{T}\right)} \\
& \leq\left|r^{\frac{\delta+(\gamma-1)-\sigma}{2}} \cdot r^{\frac{2 \gamma+2 \sigma-9}{4}} Z^{2} \dot{\Phi} \cdot r^{\frac{4 \gamma-11}{4}} Z^{3} \dot{\Phi} \cdot r^{\frac{\mu-1-\delta}{2}} \partial_{r} Z\left(Z_{2}^{2}+Z_{3}^{2}\right) \dot{\Phi}\right|_{L_{1}\left(D_{T}\right)} \\
& \leq C\left|r^{\frac{2 \gamma+2 \sigma-9}{4}} Z^{2} \dot{\Phi}\right|_{L^{4}\left(D_{T}\right)}\left|r^{\frac{4 \gamma-11}{4}} Z^{3} \dot{\Phi}\right|_{L^{4}\left(D_{T}\right)}\left|r^{\frac{\mu-1-\delta}{2}} \partial_{r} Z\left(Z_{2}^{2}+Z_{3}^{2}\right) \dot{\Phi}\right|_{L^{2}\left(D_{T}\right)}, \quad(\text { By } \delta<\sigma-(\gamma-1))
\end{aligned}
$$

then together with Lemma 2.6-Lemma 2.7 we can arrive at

$$
\int_{D_{T}}\left|M_{4}^{1} \cdot \mathcal{M} Z\left(Z_{2}^{2}+Z_{3}^{2}\right) \dot{\Phi}\right| d x \leq C \varepsilon \int_{D_{T}} \sum_{l=0}^{3}\left(r^{\mu-1-\delta+2 l}\left(\nabla_{x}^{l} \partial_{r} \dot{\Phi}\right)^{2}+r^{\mu+1-2 \gamma+2 l}\left(\nabla_{x}^{l}\left(\frac{1}{r} Z \dot{\Phi}\right)\right)^{2}\right) d x .
$$

Collecting (5.73) and (5.74) yields

$$
\int_{D_{T}}\left|M_{4} \cdot \mathcal{M} Z\left(Z_{2}^{2}+Z_{3}^{2}\right) \dot{\Phi}\right| d x \leq C \varepsilon \int_{D_{T}} \sum_{l=0}^{3}\left(r^{\mu+1-2 \gamma+2 l}\left(\nabla_{x}^{l}\left(\frac{1}{r} Z \dot{\Phi}\right)\right)^{2}+r^{\mu-1-\delta+2 l}\left(\nabla_{x}^{l} \partial_{r} \dot{\Phi}\right)^{2}\right) d x
$$

Substituting (5.67)-(5.69), (5.72) and (5.75) into (5.66) yields

$$
\begin{aligned}
& T^{\mu} \int_{S_{T}}\left(\partial_{r} Z\left(Z_{2}^{2}+Z_{3}^{2}\right) \dot{\Phi}\right)^{2} d S+T^{\mu-2 \gamma} \int_{S_{T}}\left(Z^{2}\left(Z_{2}^{2}+Z_{3}^{2}\right) \dot{\Phi}\right)^{2} d S \\
& \quad+\int_{D_{T}}\left(r^{\mu-1-\delta}\left(\partial_{r} Z\left(Z_{2}^{2}+Z_{3}^{2}\right) \dot{\Phi}\right)^{2}+r^{\mu-1-2 \gamma}\left(Z^{2}\left(Z_{2}^{2}+Z_{3}^{2}\right) \dot{\Phi}\right)^{2}\right) d x \\
& \leq C \varepsilon^{2}+C \varepsilon \int_{D_{T}} \sum_{l=0}^{3}\left(r^{\mu+1-2 \gamma+2 l}\left(\nabla_{x}^{l}\left(\frac{1}{r} Z \dot{\Phi}\right)\right)^{2}+r^{\mu-1-\delta+2 l}\left(\nabla_{x}^{l} \partial_{r} \dot{\Phi}\right)^{2}\right) d x \\
& \quad+C \varepsilon\left(T^{\mu} \int_{S_{T}}\left(\partial_{r} Z\left(Z_{2}^{2}+Z_{3}^{2}\right) \dot{\Phi}\right)^{2} d S+T^{\mu-2 \gamma} \int_{S_{T}}\left(Z^{2}\left(Z_{2}^{2}+Z_{3}^{2}\right) \dot{\Phi}\right)^{2} d S\right) .
\end{aligned}
$$

By (5.76), we have obtained the estimates of $Z^{2}\left(Z_{2}^{2}+Z_{3}^{2}\right) \dot{\Phi}$ and $\partial_{r} Z\left(Z_{2}^{2}+Z_{3}^{2}\right) \dot{\Phi}$ on $S_{T}$ or $D_{T}$. However, the related estimates on such third order derivatives $Z^{3} \dot{\Phi}$ and $\partial_{r} Z^{2} \dot{\Phi}$ are not obtained. Note that away from $\varphi=0$ in $\Omega$, as in (5.60), we can actually get the estimates of $Z^{3} \dot{\Phi}$ and $\partial_{r} Z^{2} \dot{\Phi}$ like (5.76) since

$$
\sum_{i, j, k, l=1}^{3}\left|Z_{i} Z_{j} Z_{k} Z_{l} \dot{\Phi}\right| \leq C\left(\sum_{i, k=1}^{3}\left|Z_{i} Z_{k}\left(Z_{2}^{2}+Z_{3}^{2}\right) \dot{\Phi}\right|+\sum_{i, j, k=1}^{3}\left|Z_{i} Z_{j} Z_{k} \dot{\Phi}\right|+\sum_{i=1}^{3}\left|Z_{i} Z_{j} \dot{\Phi}\right|+\sum_{i=1}^{3}\left|Z_{i} \dot{\Phi}\right|\right)
$$

and

$$
\sum_{i, j, k=1}^{3}\left|\partial_{r} Z_{i} Z_{j} Z_{k} \dot{\Phi}\right| \leq C\left(\sum_{k=1}^{3}\left|\partial_{r} Z_{k}\left(Z_{2}^{2}+Z_{3}^{2}\right) \dot{\Phi}\right|+\sum_{i, j=1}^{3}\left|\partial_{r} Z_{i} Z_{j} \dot{\Phi}\right|+\sum_{i=1}^{3}\left|\partial_{r} Z_{i} \dot{\Phi}\right|+\left|\partial_{r} \dot{\Phi}\right|\right)
$$

hold for $\varphi>\frac{\varphi_{0}}{3}$. 
Near $\varphi=0$, as in (5.62), applying Theorem 4.1 yields

$$
\begin{aligned}
& T^{\mu} \int_{S_{T}} \chi(\varphi)\left(\partial_{r} Z^{3} \dot{\Phi}\right)^{2} d S+T^{\mu-2 \gamma} \int_{S_{T}} \chi(\varphi)\left(Z^{4} \dot{\Phi}\right)^{2} d S \\
& \quad+\int_{D_{T}}\left(r^{\mu-\gamma} \chi(\varphi)\left(\partial_{r} Z^{3} \dot{\Phi}\right)^{2}+r^{\mu-1-2 \gamma} \chi(\varphi)\left(Z^{4} \dot{\Phi}\right)^{2}\right) d x \\
& \leq C\left(\int_{D_{T}} r^{\mu-1-2 \gamma}\left|\chi^{\prime}(\varphi)\right|\left(Z^{4} \dot{\Phi}\right)^{2} d x\right)^{\frac{1}{2}}\left(\int_{D_{T}} r^{\mu-\gamma}\left|\chi^{\prime}(\varphi)\right|\left(\partial_{r} Z^{3} \dot{\Phi}\right)^{2} d x\right)^{\frac{1}{2}} \\
& \quad+\int_{D_{T}} \mathcal{L} Z^{3} \dot{\Phi} \cdot \chi(\varphi) \mathcal{M} Z^{3} \dot{\Phi} d x+C \varepsilon^{2},
\end{aligned}
$$

where $\chi(\varphi)$ has been defined in (5.62), and $\chi^{\prime}(\varphi)$ has a compact support away from $\varphi=0$, which implies that the first term in the right hand side of (5.77) can be estimated as in (5.76).

By the compact support property of $\chi(\varphi)$, similar to the treatment on $\int_{D_{T}} \mathcal{L} Z\left(Z_{2}^{2}+Z_{3}^{2}\right) \dot{\Phi}$. $\mathcal{M} Z\left(Z_{2}^{2}+Z_{3}^{2}\right) \dot{\Phi} d x$ in (5.67), we then have

$$
\begin{aligned}
& \int_{D_{T}} \mathcal{L} Z^{3} \dot{\Phi} \cdot \chi(\varphi) \mathcal{M} Z^{3} \dot{\Phi} d x \\
& \leq C \varepsilon^{2}+C \varepsilon \int_{D_{T}} \sum_{l=0}^{3}\left(r^{\mu+1-2 \gamma+2 l}\left(\nabla_{x}^{l}\left(\frac{1}{r} Z \dot{\Phi}\right)\right)^{2}+r^{\mu-1-\delta+2 l}\left(\nabla_{x}^{l} \partial_{r} \dot{\Phi}\right)^{2}\right) d x \\
& \quad+C \varepsilon \sum_{l=0}^{3}\left(T^{\mu+2 l} \int_{S_{T}}\left(\nabla_{x}^{l} \partial_{r} \dot{\Phi}\right)^{2} d S+T^{\mu-2 \gamma+2 l} \int_{S_{T}}\left(\nabla_{x}^{l}\left(\frac{1}{r} Z \dot{\Phi}\right)\right)^{2} d S\right) .
\end{aligned}
$$

Substituting (5.78) into (5.77) and combining the obtained estimates for $\varphi>\frac{\varphi_{0}}{3}$ in $\Omega$, we then complete the proof of Lemma 5.5.

Based on Lemma 5.2-Lemma 5.5 and Remark 5.1-Remark 5.5, we now start to prove Theorem 5.1.

Proof of Theorem 5.1.

By (5.4) in Lemma 5.2, we have

$$
T^{\mu} \int_{S_{T}}\left(\partial_{r} \dot{\Phi}\right)^{2} d S+T^{\mu-2 \gamma} \int_{S_{T}}(Z \dot{\Phi})^{2} d S+\int_{D_{T}}\left(r^{\mu-1-\delta}\left(\partial_{r} \dot{\Phi}\right)^{2}+r^{\mu-1-2 \gamma}(Z \dot{\Phi})^{2}\right) d x \leq C \varepsilon^{2}
$$

By $\delta \leq \gamma-1$, Lemma 2.5 and (5.79), we can complete the proof of (5.2) in the case of $k=0$.

Similarly, by Lemma 5.2-Lemma 5.5, Remark 5.1-Remark 5.5, Lemma 2.5 and Remark 2.3, we can arrive at

$$
\begin{aligned}
& \sum_{l=1}^{m}\left(T^{\mu+2 l} \int_{S_{T}}\left|\nabla_{x}^{l} \partial_{r} \dot{\Phi}\right|^{2} d S+T^{\mu-2 \gamma+2 l} \int_{S_{T}}\left|\nabla_{x}^{l}\left(\frac{1}{r} Z \dot{\Phi}\right)\right|^{2} d S\right) \\
& \quad+\sum_{l=1}^{m} \int_{D_{T}}\left(r^{\mu-1-\delta+2 l}\left|\nabla_{x}^{l} \partial_{r} \dot{\Phi}\right|^{2}+r^{\mu+1-2 \gamma+2 l}\left|\nabla_{x}^{l}\left(\frac{1}{r} Z \dot{\Phi}\right)\right|^{2}\right) d x
\end{aligned}
$$




$$
\begin{aligned}
\leq & C \varepsilon^{2}+C \varepsilon\left\{\sum_{l=0}^{m}\left(T^{\mu+2 l} \int_{S_{T}}\left|\nabla_{x}^{l} \partial_{r} \dot{\Phi}\right|^{2} d S+T^{\mu-2 \gamma+2 l} \int_{S_{T}}\left|\nabla_{x}^{l}\left(\frac{1}{r} Z \dot{\Phi}\right)\right|^{2} d S\right)\right. \\
& \left.+\sum_{l=0}^{m} \int_{D_{T}}\left(r^{\mu-1-\delta+2 l}\left|\nabla_{x}^{l} \partial_{r} \dot{\Phi}\right|^{2}+r^{\mu+1-2 \gamma+2 l}\left|\nabla_{x}^{l}\left(\frac{1}{r} Z \dot{\Phi}\right)\right|^{2}\right) d x\right\} \\
& +C \varepsilon\left(\sum_{l=1}^{m} \int_{D_{T}}\left(r^{\mu-1-\delta+2 l}\left|\nabla_{x}^{l} \partial_{r} \dot{\Phi}\right|^{2}+r^{\mu+1-2 \gamma+2 l}\left|\nabla_{x}^{l}\left(\frac{1}{r} Z \dot{\Phi}\right)\right|^{2}\right) d x\right)^{\frac{1}{2}} .
\end{aligned}
$$

Then for $1 \leq m \leq 3$, it follows from (5.79)-(5.80) that

$$
\begin{aligned}
& \sum_{l=1}^{m}\left(T^{\mu+2 l} \int_{S_{T}}\left|\nabla_{x}^{l} \partial_{r} \dot{\Phi}\right|^{2} d S+T^{\mu-2 \gamma+2 l} \int_{S_{T}}\left|\nabla_{x}^{l}\left(\frac{1}{r} Z \dot{\Phi}\right)\right|^{2} d S\right) \\
& \quad+\sum_{l=1}^{m} \int_{D_{T}}\left(r^{\mu-1-\delta+2 l}\left|\nabla_{x}^{l} \partial_{r} \dot{\Phi}\right|^{2}+r^{\mu+1-2 \gamma+2 l}\left|\nabla_{x}^{l}\left(\frac{1}{r} Z \dot{\Phi}\right)\right|^{2}\right) d x \\
& \leq C \varepsilon^{2}+C \varepsilon\left(\sum_{l=1}^{m} \int_{D_{T}} r^{\mu-1-\delta+2 l}\left|\nabla_{x}^{l} \partial_{r} \dot{\Phi}\right|^{2}+r^{\mu+1-2 \gamma+2 l}\left|\nabla_{x}^{l}\left(\frac{1}{r} Z \dot{\Phi}\right)\right|^{2} d x\right)^{\frac{1}{2}} .
\end{aligned}
$$

If

$$
\sum_{l=1}^{m} \int_{D_{T}} r^{\mu-1-\delta+2 l}\left|\nabla_{x}^{l} \partial_{r} \dot{\Phi}\right|^{2}+r^{\mu+1-2 \gamma+2 l}\left|\nabla_{x}^{l}\left(\frac{1}{r} Z \dot{\Phi}\right)\right|^{2} d x \leq C \varepsilon^{2},
$$

then (5.2) is derived directly;

If

$$
\sum_{l=1}^{m} \int_{D_{T}} r^{\mu-1-\delta+2 l}\left|\nabla_{x}^{l} \partial_{r} \dot{\Phi}\right|^{2}+r^{\mu+1-2 \gamma+2 l}\left|\nabla_{x}^{l}\left(\frac{1}{r} Z \dot{\Phi}\right)\right|^{2} d x \geq C \varepsilon^{2},
$$

then it follows from (5.81) that

$$
\begin{aligned}
& \sum_{l=1}^{m} \int_{D_{T}}\left(r^{\mu-1-\delta+2 l}\left|\nabla_{x}^{l} \partial_{r} \dot{\Phi}\right|^{2}+r^{\mu+1-2 \gamma+2 l}\left|\nabla_{x}^{l}\left(\frac{1}{r} Z \dot{\Phi}\right)\right|^{2}\right) d x \\
\leq & C \varepsilon\left(\sum_{l=1}^{m} \int_{D_{T}} r^{\mu-1-\delta+2 l}\left|\nabla_{x}^{l} \partial_{r} \dot{\Phi}\right|^{2}+r^{\mu+1-2 \gamma+2 l}\left|\nabla_{x}^{l}\left(\frac{1}{r} Z \dot{\Phi}\right)\right|^{2} d x\right)^{\frac{1}{2}},
\end{aligned}
$$

which means

$$
\sum_{l=1}^{m} \int_{D_{T}}\left(r^{\mu-1-\delta+2 l}\left|\nabla_{x}^{l} \partial_{r} \dot{\Phi}\right|^{2}+r^{\mu+1-2 \gamma+2 l}\left|\nabla_{x}^{l}\left(\frac{1}{r} Z \dot{\Phi}\right)\right|^{2}\right) d x \leq C \varepsilon^{2} .
$$

Substituting this into (5.81) derives (5.2) for $1 \leq k \leq 3$ and further completes the proof of Theorem 5.1. 
It follows from Sobolev's embedding theorem (see also [10, Lemma 14]) that, one has for $1 \leq r \leq T$

$$
\left\{\begin{array}{l}
\sum_{0 \leq l \leq 1}\left|r^{l} \nabla_{x}^{l}\left(\partial_{r} \dot{\Phi}\right)\right|^{2} \leq C r^{-2} \int_{S_{r}} \sum_{0 \leq l \leq 3}\left|r^{l} \nabla_{x}^{l}\left(\partial_{r} \dot{\Phi}\right)\right|^{2} d S, \\
\sum_{0 \leq l \leq 1}\left|r^{l} \nabla_{x}^{l}\left(\frac{1}{r} Z \dot{\varphi}\right)\right|^{2} \leq C r^{-2} \int_{S_{r}} \sum_{0 \leq l \leq 3}\left|r^{l} \nabla_{x}^{l}\left(\frac{1}{r} Z \dot{\varphi}\right)\right|^{2} d S .
\end{array}\right.
$$

On the other hand, (5.2) shows that

$$
\int_{S_{r}} \sum_{0 \leq l \leq 3}\left|r^{l} \nabla_{x}^{l}\left(\partial_{r} \dot{\Phi}\right)\right|^{2} d S \leq C \varepsilon^{2} r^{-\mu}, \quad \int_{S_{r}} \sum_{0 \leq l \leq 3}\left|r^{l} \nabla_{x}^{l}\left(\frac{1}{r} Z \dot{\Phi}\right)\right|^{2} d S \leq C \varepsilon^{2} r^{-\mu+2 \gamma-2} .
$$

Hence we arrive at

$$
\sum_{0 \leq l \leq 1}\left|r^{l} \nabla_{x}^{l}\left(\partial_{r} \dot{\Phi}\right)\right|^{2} \leq C \varepsilon^{2} r^{-\mu-2}, \quad \sum_{0 \leq l \leq 1}\left|r^{l} \nabla_{x}^{l}\left(\frac{1}{r} Z \dot{\Phi}\right)\right|^{2} \leq C \varepsilon^{2} r^{-\mu+2 \gamma-4} .
$$

Subsequently, one has

$$
\sum_{0 \leq l \leq 1}\left|r^{l} \nabla_{x}^{l}\left(\partial_{r} \dot{\Phi}\right)\right| \leq C \varepsilon r^{-\frac{\mu+2}{2}}=C \varepsilon r^{-2(\gamma-1)}, \quad \sum_{0 \leq l \leq+1}\left|r^{l} \nabla_{x}^{l}\left(\frac{1}{r} Z \dot{\Phi}\right)\right| \leq C \varepsilon r^{-\frac{\mu-2 \gamma+4}{2}}=C \varepsilon r^{-(\gamma-1)}
$$

In addition, for $1<\gamma<2$,

$$
|Z \dot{\Phi}| \leq|Z \dot{\Phi}(1, \varphi)|+\int_{1}^{r}\left|\partial_{r} Z \dot{\Phi}(t, \varphi)\right| d t \leq C \varepsilon\left(1+r^{1-2(\gamma-1)}\right),
$$

which means

$$
\frac{1}{r}|Z \dot{\Phi}| \leq C \varepsilon\left(r^{-1}+r^{-2(\gamma-1)}\right) \leq C \varepsilon r^{-\sigma}
$$

In this case, by the Bernoulli's law (1.2), we have $c^{2}(\rho)=c^{2}(\hat{\rho})-\frac{\gamma-1}{2}\left(\left(\partial_{r} \dot{\Phi}\right)^{2}+2 \hat{U} \partial_{r} \dot{\Phi}+\right.$ $\left.\frac{1}{r^{2}}(Z \dot{\Phi})^{2}\right)$, which derives $C r^{2(1-\gamma)}-C \varepsilon\left(r^{2(1-\gamma)}+r^{-2 \sigma}\right)<c^{2}(\rho)<C r^{2(1-\gamma)}+C \varepsilon\left(r^{2(1-\gamma)}+\right.$ $\left.r^{-2 \sigma}\right)$ together with Lemma 2.1. Thus, one obtains $c^{2}(\rho) \sim r^{2(1-\gamma)}>0$ for any $r \geq 1$ and small $\varepsilon$. Therefore, the proof of Theorem 1.1 is completed by the local existence result in Theorem 3.1 and continuous induction method, where the $C^{\infty}$ regularity of $\Phi$ comes from the strong continuity principle (see [19]) and the $C^{\infty}$ smoothness of initial data $\left(\left.\Phi(x)\right|_{r=1},\left.\partial_{r} \Phi(x)\right|_{r=1}\right)$ and boundary $\Sigma$.

\section{References}

[1] S. Alinhac, Temps de vie des solutions régulières des équations d'Euler compressibles axisymétriques en dimension deux, Invent. Math. 111, no. 3, 627-670 (1993).

[2] S. Alinhac, Blowup of small data solutions for a class of quasilinear wave equations in two space dimensions. II, Acta Math. 182, no. 1, 1-23 (1999). 
[3] J. Y. Chemin, Dynamique des gaz à masse totale finie, Asymptotic Anal. 3, no. 3, 215-220 (1990).

[4] D. Christodoulou, Global solutions of nonlinear hyperbolic equations for small initial data, Comm. Pure Appl. Math. 39, no. 2, 267-282 (1986).

[5] R. Courant, K. O. Friedrichs, Supersonic flow and shock waves, Interscience Publishers Inc., New York, 1948.

[6] D. Coutand, S. Shkoller, Well-posedness in smooth function spaces for moving-boundary 1-D compressible Euler equations in physical vacuum, Comm. Pure Appl. Math. 64, no. 3, 328-366 (2011).

[7] D. Coutand, S. Shkoller, Well-posedness in smooth function spaces for the movingboundary three-dimensional compressible Euler equations in physical vacuum, Arch. Ration. Mech. Anal. 206, no. 2, 515-616 (2012).

[8] M. D’Abbicco, S. Lucente, M. Reissig, Semi-linear wave equations with effective damping, Chin. Ann. Math. Ser. B 34, no. 3, 345-380 (2013).

[9] P. Godin, The lifespan of a class of smooth spherically symmetric solutions of the compressible Euler equations with variable entropy in three space dimensions, Arch. Ration. Mech. Anal. 177, no. 3, 479-511 (2005).

[10] P. Godin, Global shock waves in some domains for the isentropic irrotational potential flow equations, Comm. Partial Differential Equations 22, 1929-1997 (1997).

[11] M. Grassin, Global smooth solutions to Euler equations for a perfect gas, Indiana Univ. Math. J. 47, no. 4, 1397-1432 (1998).

[12] J. Jang, N. Masmoudi, Well-posedness for compressible Euler equations with physical vacuum singularity, Comm. Pure Appl. Math. 62, no. 10, 1327-1385 (2009).

[13] J. Jang, N. Masmoudi, Well-posedness of compressible Euler equations in a physical vacuum, arXiv:1005.4441, Preprint, 2010.

[14] S. Klainerman, The null condition and global existence to nonlinear wave equations, Nonlinear systems of partial differential equations in applied mathematics, Part 1 (Santa Fe, N.M., 1984), 293-326, Lectures in Appl. Math., 23, Amer. Math. Soc., Providence, RI, 1986.

[15] Li Jun, Ingo Witt, Yin Huicheng, Global multidimensional shock waves for 2-D and 3-D unsteady potential flow equations, arXiv:1310.3470 (2013).

[16] Li Jun, Xin Zhouping, Yin Huicheng, Transonic shocks for the full compressible Euler system in a general two-dimensional de Laval nozzle, Arch. Ration. Mech. Anal. 207, no. 2, 533-581 (2013). 
[17] Lin Changshou, Interpolation inequalities with weights, Comm. Partial Differential Equations 11, no. 14, 1515-1538 (1986).

[18] Liu Tai-Ping, Xin Zhouping, Yang Tong, Vacuum states for compressible flow, Discrete Contin. Dynam. Systems 4, no. 1, 1-32 (1998).

[19] A. Majda, Compressible fluid flow and systems of conservation laws in several space variables, Applied Mathematical Sciences, 53. Springer-Verlag, New York, 1984.

[20] T. Makino, S. Ukai, S. Kawashima, Sur la solution à support compact de l'équations d'Euler compressible, Japan J. Appl. Math. 3, no. 2, 249-257 (1986).

[21] M. Rammaha, Formation of singularities in compressible fluids in two-space dimensions, Proc. Amer. Math. Soc. 107, 705-714 (1989).

[22] D. Serre, Solutions classiques globales des équations d'Euler pour un fluide parfait compressible, Ann. Inst. Fourier (Grenoble) 47, no. 1, 139-153 (1997)

[23] J. Wirth, Wave equations with time-dependent dissipation. I. Non-effective dissipation, J. Differential Equations 222, no. 2, 487-514 (2006).

[24] J. Wirth, Wave equations with time-dependent dissipation. II. Effective dissipation, J. Differential Equations 232, no. 1, 74-103 (2007).

[25] Xin Zhouping, Blowup of smooth solutions to the compressible Navier-Stokes equation with compact density, Comm. Pure Appl. Math. 51, no. 3, 229-240 (1998).

[26] Xu Chao-Jiang, Yang Tong, Local existence with physical vacuum boundary condition to Euler equations with damping, J. Differential Equations 210, no. 1, 217-231 (2005).

[27] Yang Tong, Singular behavior of vacuum states for compressible fluids, J. Comput. Appl. Math. 190, no. 1-2, 211-231 (2006).

[28] Yin Huicheng, Formation and construction of a shock wave for 3-D compressible Euler equations with the spherical initial data, Nagoya Math. J. 175, 125-164 (2004). 